\title{
Modellbildung für die Schallverstärkung in nachgiebig ausgekleideten Strömungskanälen
}

\author{
Dissertation \\ zur Erlangung des Doktorgrades \\ der Mathematisch-Naturwissenschaftlichen Fakultäten \\ der Georg-August-Universität zu Göttingen
}

vorgelegt von
Jakob Großer
geboren in
Plauen

Plauen

Göttingen 2003 
D 7

Referent: Prof. Dr. Dirk Ronneberger

Koreferent: Prof. Dr. Andreas Tilgner

Tag der Disputation: 4. November 2003 


\section{Bezeichnungen}

In dieser Arbeit werden Vektoren mit fetten Buchstaben, der Wellenvektor etwa mit $\mathbf{k}$, Beträge mit einfachen Buchstaben, $|\mathbf{k}|=k$, und Komponenten in der Weise $k_{x}, k_{y}, k_{z}$ gekennzeichnet.

Mit Betragsstrichen |.| bezeichne ich sowohl den Betrag reeller Vektorgrößen

$$
|\mathbf{r}|=\sqrt{r_{x}^{2}+r_{y}^{2}+r_{z}^{2}}
$$

als auch den Absolutbetrag komplexer Zahlen

$$
|z|=\sqrt{(\operatorname{Re} z)^{2}+(\operatorname{Im} z)^{2}}
$$

Für die Phasen wellenartiger Größen verwende ich mit Ausnahme von Anhang C die Konvention $\exp (-i \omega t+i \mathbf{k x})$.

In Anhang $\mathrm{C}$ wähle ich stattdessen wie in [10] $\exp (i \omega t+i \mathbf{k x})$, wobei sich die Phasenfronten dann entgegengesetzt zum Vektor $\mathbf{k}$ ausbreiten. Hierdurch können die Berechnungen für einen Kanal mit kreisförmigem Querschnitt direkt mit den dort abgeleiteten Formeln verglichen werden.

FOURIER- und LAPLACE-Transformierte werde ich der Einfachheit halber mit denselben Buchstaben wie die Ausgangsfunktion kennzeichen. Die räumliche FOURIER-Transformierte eines Feldes $\Phi(x)$ ist dann einfach $\Phi\left(k_{x}\right)$.

Stationäre Grundströmungen laufen in dieser Arbeit immer von $x<0$ nach $x>0$ (nach rechts). Stromab oder in Grundströmungsrichtung laufende Wellen breiten sich daher (eine Quelle bei $x=0$ angenommen) in den Halbraum $x>0$ aus. Analog werde ich die Bezeichnungen stromauf beziehungsweise entgegen der Grundströmung benutzen. 


\section{Symbole und Bezeichnungen}

\begin{tabular}{|c|c|}
\hline$B$ & Kammerbreite der Wandauskleidung \\
\hline$c$ & Schallgeschwindigkeit: $343 \mathrm{~m} \cdot \mathrm{s}^{-1}$ \\
\hline$D$ & $\begin{array}{l}\text { Kanaldurchmesser: } 50 \mathrm{~mm} \text {, im Falle des Flachkanals: } \\
\text { Kanalbreite }\end{array}$ \\
\hline$\partial_{x}, \partial_{y}^{2}$ usw & $\frac{\partial}{\partial x}, \frac{\partial^{2}}{\partial y^{2}}$ usw. \\
\hline $\mathbf{e}_{x}, \mathbf{e}_{y}, \mathbf{e}_{z}$ & Einheitsvektoren in $x$-, $y$ - und z-Richtung \\
\hline$\vec{E}_{s}$ & Amplitude der stromab einfallenden Welle \\
\hline$\overleftarrow{E}_{s}$ & Amplitude der stromauf einfallenden Welle \\
\hline$f$ & Frequenz \\
\hline$f_{0}$ & Resonanzfrequenz der Wandauskleidung: $849 \mathrm{~Hz}$ \\
\hline$K$ & $\begin{array}{l}\text { kompressible HELMHOLTZ-Zahl: } \\
K=\omega L_{\mathrm{ref}} /\left(c \sqrt{1-M a^{2}}\right)\end{array}$ \\
\hline$K_{n}^{m}$ & $\begin{array}{l}\text { Querwellenzahl der Mode n-ter Ordnung im Rohr bei } \\
\text { Winkelabhängigkeit } \exp ( \pm i m \phi)\end{array}$ \\
\hline k & Wellenvektor \\
\hline$k_{\mathrm{St}}$ & wandnormale Wellenzahlkomponente der STOKES-Welle \\
\hline$L, l_{0}, l$ & $\begin{array}{l}\text { Länge des nachgiebig ausgekleideten Kanalstücks: } \\
87,5 \mathrm{~mm}\end{array}$ \\
\hline $\mathcal{L}$ & $\begin{array}{l}\text { Funktion aus der WIENER-Hopf-Methode, deren Null- } \\
\text { bzw. Polstellen die Ausbreitungswellenzahlen der Eigen- } \\
\text { moden des nachgiebigen bzw. schallharten Kanalstückes } \\
\text { sind }\end{array}$ \\
\hline $\mathcal{L}_{\oplus}$ & $\begin{array}{l}\text { Plus-Faktor von L, in dem die Stromauf-Moden enthal- } \\
\text { ten sind }\end{array}$ \\
\hline $\mathcal{L}_{\ominus}$ & $\begin{array}{l}\text { Minus-Faktor von L, in dem die Stromab-Moden enthal- } \\
\text { ten sind }\end{array}$ \\
\hline$L_{\text {ref }}$ & Referenzlänge aus Anhang C: Kanaldurchmesser \\
\hline$M a$ & MACH-Zahl \\
\hline$P_{n}$ & Hilfsgröße aus Anhang C \\
\hline$p, p_{0}$ & Wechseldruck(feld), statischer Luftdruck \\
\hline$Q_{n}$ & Hilfsgröße aus Anhang C \\
\hline
\end{tabular}




\begin{tabular}{|c|c|}
\hline$R, R_{0}$ & Kanalradius: $25 \mathrm{~mm}$ \\
\hline$R_{a}$ & Kammerradius: $100 \mathrm{~mm}$ \\
\hline$R_{\leftarrow}$ & $\begin{array}{l}\text { Reflexionsfaktor bei Beschallung stromab; } R_{a} \text { bei } \\
\text { BRANDES }\end{array}$ \\
\hline$R_{\rightarrow}$ & $\begin{array}{l}\text { Reflexionsfaktor bei Beschallung stromauf; } R_{b} \text { bei } \\
\text { BRANDES }\end{array}$ \\
\hline$\overleftarrow{R}_{m, n}$ & $\begin{array}{l}\text { Amplituden der Moden aus Anhang } \mathrm{C} \text {, die das Objekt } \\
\text { stromauf verlassen; entsprechen für } m=n=0 R_{\leftarrow} \text { bzw. } \\
T_{\leftarrow}\end{array}$ \\
\hline$R_{n}$ & Hilfsgröße aus Anhang C \\
\hline$r$ & Ortskoordinate in radialer Richtung \\
\hline$S_{\oplus}, S_{\ominus}$ & $\begin{array}{l}\text { Halbebenen, auf denen Plus- bzw. Minus-Funktionen } \\
\text { holomorph sind }\end{array}$ \\
\hline$S_{n}$ & Hilfsgröße aus Anhang C \\
\hline$T$ & Kammertiefe der Wandauskleidung \\
\hline$T_{\rightarrow}$ & $\begin{array}{l}\text { Transmissionsfaktor bei Beschallung stromab; } T_{a} \text { bei } \\
\text { BRANDES }\end{array}$ \\
\hline$T_{\leftarrow}$ & $\begin{array}{l}\text { Transmissionsfaktor bei Beschallung stromauf; } T_{b} \text { bei } \\
\text { BRANDES }\end{array}$ \\
\hline$\vec{T}_{m, n}$ & $\begin{array}{l}\text { Amplituden der Moden aus Anhang } \mathrm{C} \text {, die das Objekt } \\
\text { stromab verlassen; entsprechen für } m=n=0 T_{\rightarrow} \text { bzw. } \\
R_{\rightarrow}\end{array}$ \\
\hline $\mathbf{u}$ & Vektor(feld) der Gleichströmungsgeschwindigkeit \\
\hline$u, u_{0}$ & Gleichströmungsgeschwindigkeit (Rechteckprofil) \\
\hline $\bar{u}$ & $\begin{array}{l}\text { über den Querschnitt gemittelte Gleichströmungsge- } \\
\text { schwindigkeit }\end{array}$ \\
\hline$u_{\tau}$ & $\begin{array}{l}\text { Schubspannungsgeschwindigkeit, gebildet mit der } \\
\text { Wandschubspannung } \tau_{\mathrm{w}}: u_{\tau}=\sqrt{\tau_{\mathrm{w}} / \rho}\end{array}$ \\
\hline$u_{\oplus}^{*}, u_{\ominus}^{*}$ & Hilfsgrößen aus der Streurechnung \\
\hline $\mathbf{v}$ & Vektor(feld) der Wechselgeschwindigkeit (Schnelle) \\
\hline$v_{\mathrm{ph}}$ & Geschwindigkeit der Fronten gleicher Phase \\
\hline$x$ & Ortskoordinate in Ausbreitungsrichtung \\
\hline $\begin{array}{l}y \\
z\end{array}$ & Ortskoordinate senkrecht zur ausgekleideten Wand \\
\hline & \\
\hline
\end{tabular}


$Z, Z^{\prime} \quad$ Wandimpedanz ohne bzw. mit dem Faktor $\omega^{\prime} / \omega$

$\Delta_{m, \sigma} \quad$ Hilfsgröße: Faktor aus $\vec{T}_{m, \sigma}, \overleftarrow{R}_{m, \sigma}$

$\gamma_{n}$

Polstellen von $\mathcal{L}$; Ausbreitungswellenzahlen der Eigenmoden im schallharten Kanal

$\eta \quad$ Auslenkung kleiner Fluidvolumina, speziell: Auslenkung der Wirbelschicht

$\kappa \quad$ KÁrmán-Konstante: 0,4

$\lambda_{n}^{ \pm} \quad$ Nullstellen von $\mathcal{L}:$ Ausbreitungswellenzahlen der Eigenmoden im nachgiebigen Kanalstück

$\nu \quad$ kinematische Zähigkeit der Luft: $1,51 \cdot 10^{-5} \mathrm{~m}^{2} \cdot \mathrm{s}^{-1}$

$\nu_{\mathrm{t}} \quad$ scheinbare turbulente Zähigkeit

$\rho, \rho_{0} \quad$ Wechselanteil der Luftdichte; Luftdichte: $1,2 \mathrm{~kg} \cdot \mathrm{m}^{-3}$

$\Phi$

irgendein Wechselfeld, speziell: Potential der Wechselgeschwindigkeit

$\Phi_{\oplus}, \Phi_{\ominus} \quad$ Halbseitige FouRIER-Transformierte einer Feldgröße

$\phi_{m}, \psi_{m} \quad$ Nahfelder aus Anhang C

$\phi \quad$ Winkelkoordinate im Rohrkanal

$\omega \quad$ Kreisfrequenz

$\omega_{0} \quad$ Resonanz-Kreisfrequenz der Wandauskleidung:

$2 \pi \cdot 849 \mathrm{~Hz}$

$\omega^{\prime} \quad$ DoppleR-verschobene Kreisfrequenz, $\omega^{\prime}=\omega-u k_{x}$ 


\section{Abbildungsverzeichnis}

2.1 Aufbau des Strömungskanals zur Messung von Streufaktoren. An die Stelle des Kanalstückes können unterschiedliche Objekte eingebaut werden. . . . . . . . . . . . . . 5 5

2.2 Bezeichnung der Streufaktoren . . . . . . . . . . . . . . 6

2.3 Schnitt durch ein Meßobjekt (nicht maßstäblich) . . . . . . . . . 7

2.4 Beträge des Transmissions- und des Reflexionsfaktors bei Beschallung in Stromab-Richtung. Aus: Diss. Brandes . . . . . . . . . . 9

2.5 Beträge des Transmissions- und des Reflexionsfaktors bei Beschallung stromauf. Die Abweichungen von $R_{\rightarrow}$ vom generellen Verlauf bei ca. $1100 \mathrm{~Hz}$ sind nicht signifikant (große Meßunsicherheit, siehe Diss. Brandes). . . . . . . . . . . . . . . . . . . . . . 10

2.6 Phase des Transmissionsfaktors bei Beschallung stromab. Aus: Diss. Brandes . . . . . . . . . . . . . . . . . . . . . . . . . 11

2.7 Maximalverstärkung in Abhängigkeit von der MACH-Zahl für verschiedene Kammergeometrien. Die Bauteillänge ist bei allen drei Kanalstücken gleich. Die Abbildungen sind nicht maßstäblich. Aus: Diss. BRAnDES . . . . . . . . . . . . . . . . . . . . . . . . 12

2.8 Maximalverstärkung in Abhängigkeit von der MACH-Zahl für verschiedene Bauteillängen. Aus: Diss. Brandes . . . . . . . . . . . 13

3.1 Betrag und Phase der Impedanz $Z / \rho c$ einer Wand, die lokal wie ein $\lambda$ /4-Resonator nachgibt. $T=101 \mathrm{~mm} . \quad \ldots . . . . .$.

3.2 Die vier Lösungen $k_{x}^{ \pm( \pm)}$in Abhängigkeit von der Frequenz; Strömungsgeschwindigkeit $60 \mathrm{~m} \cdot \mathrm{s}^{-1}$. Grau unterlegt ist der Bereich, in dem eine Mode nicht mehr an der Wand lokalisiert ist $\left(\operatorname{Im} k_{y} \leq 0\right)$. . . . . . . . . . . . . . . . . . . . . .

3.3 Betrag von $v_{\mathrm{ph}}$ für die vier Lösungen bei einer Gleichströmung von $60 \mathrm{~m} \cdot \mathrm{s}^{-1} \ldots \ldots \ldots \ldots . \ldots . \ldots . \ldots . \ldots 23$ 
3.4 Wellenzahlen der vier Lösungen in Abhängigkeit von der Anfachungsrate $\operatorname{Im} \omega$ (kompressible Rechnung) . . . . . . . . . . . .

3.5 Der räumliche Anfachungsfaktor der Instabilitätsmode in Abhängigkeit von Frequenz und МACH-Zahl . . . . . . . . . . 27

3.6 Vergleich der kompressiblen (dicke Linien) und inkompressiblen Lösungen (dünne Linien) bei $u=90 \mathrm{~m} \cdot \mathrm{s}^{-1} \ldots \ldots$. . . . . . .

3.7 Vergleich der inkompressiblen Lösungen im Rohr (dicke Linien) mit den Moden $k_{x}^{ \pm( \pm)}$(dünne Linien) bei $u=60 \mathrm{~m} \cdot \mathrm{s}^{-1} \ldots$. . .

3.8 Wechseldruckamplitude der instabilen Mode in Abhängigkeit vom Wandabstand für drei Frequenzen, $u=60 \mathrm{~m} \cdot \mathrm{s}^{-1} \ldots \ldots \ldots$.

3.9 Die Instabilitätsmode im Rohr in Abhängigkeit von Frequenz und MACH-Zahl, kompressible Rechnung . . . . . . . . . . . . 35

3.10 Wellenzahlen der instabilen und eine weitere Mode unterhalb (oberes Bild), nahe und oberhalb (unteres Bild) der kritischen Strömungsgeschwindigkeit von etwa $70 \mathrm{~m} \cdot \mathrm{s}^{-1}$ in Abhängigkeit von der Frequenz . . . . . . . . . . . . . . . . .

3.11 Vergleich der inkompressiblen, symmetrischen Lösungen im Flachkanal (dicke Linien) mit den Lösungen im Rohr (dünne Linien) bei $u=60 \mathrm{~m} \cdot \mathrm{s}^{-1}$. Breite des Flachkanals: $D=50 \mathrm{~mm}$. . . . . . . .

3.12 Vergleich der antisymmetrischen Lösungen im Flachkanal (dicke Linien) mit den symmetrischen (dünne Linien) bei $u=60 \mathrm{~m} \cdot \mathrm{s}^{-1}$. Breite des Flachkanals: $D=50 \mathrm{~mm} . \quad \ldots \ldots \ldots \ldots$

3.13 Ausbreitungswellenzahlen der Instabilitätsmode (grün) und der ersten höheren, stromab laufenden Mode (rot) im Rohr für sinkende Strömungsgeschwindigkeit, beginnend bei $60 \mathrm{~m} \cdot \mathrm{s}^{-1} \ldots \ldots$. . .

3.14 Betrag von $v_{\text {ph }}$ für die sechs Lösungen niedrigster Ordnung im Rohr bei einer Gleichströmung von $60 \mathrm{~m} \cdot \mathrm{s}^{-1} \ldots \ldots \ldots$. . . 44

3.15 Hypothetischer Verlauf des Imaginärteils der instabilen Mode . . . 45

3.16 Radiale Abhängigkeit der turbulenten Viskosität für $u_{\tau}=2,67 \mathrm{~m}$. $\mathrm{s}^{-1}\left(\right.$ entspricht $\left.\bar{u}=60 \mathrm{~m} \cdot \mathrm{s}^{-1}\right) \ldots \ldots \ldots \ldots \ldots$

3.17 Über den Querschnitt gemittelte Grundströmungsgeschwindigkeit in Abhängigkeit von der Schubspannungsgeschwindigkeit . . . . .

3.18 Instabilitätsmode ohne (dünne Linien) und mit turbulenter Zähigkeit $\nu_{\mathrm{t}}=300 \nu$ (dicke Linien) bei $u=60 \mathrm{~m} \cdot \mathrm{s}^{-1} \ldots \ldots \ldots$.

3.19 Vergleich der Lösungen im Rohr unter Berücksichtigung der Kompressibilität und einer Zähigkeit von $\nu_{\mathrm{t}}=300 \nu$ (dicke Linien) mit den herkömmlichen Lösungen (dünne Linien) bei $u=60 \mathrm{~m} \cdot \mathrm{s}^{-1}$. 
4.1 Vergleich der Lösungen im Rohr mit Rechteckprofil (dünne Linien) und dem logarithmischen Profil (dicke Linien) bei $\bar{u}=60 \mathrm{~m} \cdot \mathrm{s}^{-1}$.

4.2 Wellenzahlen der Instabilitätsmode für komplexe Frequenzen $f$, $34 \mathrm{~Hz}<\left|f-f_{0}\right|<49 \mathrm{~Hz}$ und $\arg \left(f-f_{0}\right)=-0,18 \pi \ldots 1,92 \pi$ bei $\bar{u}=60 \mathrm{~m} \cdot \mathrm{s}^{-1} \ldots \ldots \ldots \ldots \ldots$

4.3 Wechseldruckamplitude bei $\bar{u}=60 \mathrm{~m} \cdot \mathrm{s}^{-1}$ und $900 \mathrm{~Hz}$ in Abhängigkeit vom Wandabstand. Rechnung mit Logarithmusprofil (dicke Linien) und Rechteckprofil (dünne Linien) . . . . . . . . . . . . .

4.4 Wechseldruckamplitude bei $\bar{u}=60 \mathrm{~m} \cdot \mathrm{s}^{-1}$ und $900 \mathrm{~Hz}$ in Abhängigkeit vom Wandabstand . . . . . . . . . . . . . 66

5.1 Ein stückweise homogenes Randwertproblem . . . . . . . . . . . . 69

5.2 Von Koch, MöHring untersuchte Anordnung . . . . . . . . . . . 73

5.3 Streufaktoren für ein Meßobjekt der Länge $l_{0}=8,75 \mathrm{~cm}$, Kanalbreite $h_{0}=5 \mathrm{~cm}$ und MACH-Zahl 0,16, verglichen mit den experimentellen Daten im Rohr . . . . . . . . . . . . . . . . . . 77

5.4 Phase des Transmissionsfaktors $T_{\rightarrow}$ aus der vorherigen Abb. . . . 78

5.5 Ein nachgiebig ausgekeidetes Rohrsegment . . . . . . . . . . . . . 79

5.6 Streufaktoren für ein Rohrstück der Länge $l_{0}=8,75 \mathrm{~cm}$, Durchmesser $h_{0}=5 \mathrm{~cm}$, MACH-Zahl 0,16, verglichen mit den experimentellen Daten. . . . . . . . . . . . . . . . . . . . . . 79

5.7 Phase des Transmissionsfaktors $T_{\rightarrow}$ im Rohr. . . . . . . . . . . . . 80

5.8 Streufaktoren für ein Rohrstück, aber mit $\eta(0)=\eta(l)=0, \mathrm{MACH}-$ Zahl 0,16. Die Oszillation bei $1100 \mathrm{~Hz}$ ist offenbar ein Artefakt, das kleiner wird, wenn man die Zahl der Moden erhöht-deswegen hier Berücksichtigung von achtzehn statt vierzehn Moden. . . . .

5.9 Betrag des Transmissionsfaktors $T \rightarrow$ als Funktion der Frequenz und der Machzahl. Sprungbedingungen $\eta(0)=0 ; \eta(l)$ sind so gewählt, daß Druck und Auslenkung bei $x=l$ endlich bleiben. . . . . . . . 83

A.1 Wellenzahlen der hydrodynamischen Moden für Re $f=900 \mathrm{~Hz}$ fest in Abhängigkeit vom Imaginärteil der Kreisfrequenz $\omega$. Der Imaginärteil von $k_{x}^{+(+)}$wechselt das Vorzeichen, $k_{x}^{-(+)}$dagegen bleibt stromab räumlich gedämpft. . . . . . . . . . . . . . . .

A.2 Wellenzahlen der vier Lösungen in Abhängigkeit von der Anfachungsrate Im $\omega$. dicke Linien: inkompressibel, dünne Linien: kompressibel ...................... 
B.1 Streuung am Übergang in ein anderes Medium. $c_{ \pm}$ist die Phasengeschwindigkeit in den Gebieten $x>0$ bzw. $x<0 \ldots \ldots \ldots \ldots 94$

B.2 Die Halbebenen $S_{\oplus}$ und $S_{\ominus} \ldots \ldots \ldots \ldots \ldots$

B.3 Integrationsweg für $\Phi(x<0) \ldots \ldots \ldots \ldots \ldots$

C.1 Bezeichnungen und Koordinaten für die Rohrgeometrie . . . . . . 101 


\section{Inhaltsverzeichnis}

1 Einleitung 1

2 Experiment 4

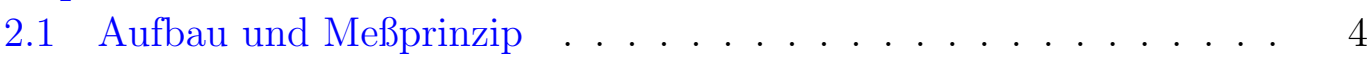

2.1.1 Der Kanal . . . . . . . . . . . . . . . . . . . . . . 4

2.1.2 Das Meßprinzip . . . . . . . . . . . . . . . . 5

2.1.3 Die Meßobjekte . . . . . . . . . . . . . 6

2.2 Die Instabilität Typ I . . . . . . . . . . . . . . . . . . . . . . . . . 8

2.2.1 Streufaktoren ............... 8

2.2.2 Abhängigkeit der Verstärkung von den experimentellen Parametern . . . . . . . . . . . . . . . 11

2.3 Vorläufige Interpretation . . . . . . . . . . . . . . . . 13

3 Einfache Modelle $\quad \mathbf{1 5}$

3.1 Inkompressibel überströmte Wand; Rechteckprofil . . . . . . . . . 15

3.1.1 Die Grundgleichungen . . . . . . . . . . . . . . . . . 16

3.1.2 Modellierung der Wandimpedanz . . . . . . . . . . . . . 17

3.1.3 Lösung der Dispersionsbeziehung; Verlauf der Ausbrei-

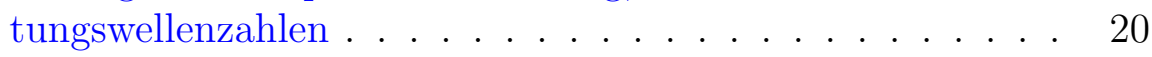

3.1.4 Diskussion der Lösungen . . . . . . . . . . . . . . . . 21

3.1.5 Die Ausbreitungsrichtung der Moden . . . . . . . . . . . . 24

3.1.6 Zusammenfassung . . . . . . . . . . . . . 26

3.2 Kompressibilität; Andere Geometrien . . . . . . . . . . . . . . 28

3.2.1 Effekte der Kompressibilität . . . . . . . . . . . . . . . 28

3.2.2 Kanal mit kreisförmigem Querschnitt . . . . . . . . . . . . 30

3.2.3 Flachkanal ................... 37 
3.2.4 Zum Begriff der hydrodynamischen Mode . . . . . . . . . . 41

3.2.5 Zusammenfassung................. 43

3.3 Zähigkeitseffekte . . . . . . . . . . . . . . . 46

3.3.1 Die Größe der turbulenten Zähigkeit . . . . . . . . . . . . 46

3.3.2 Ebene Wand. . . . . . . . . . . . . . . . . . . . . . 48

3.3 .3 Rohr ..................... . . 51

3.3.4 Verlauf der Moden für kompressible Rechnung im Rohr . . 54

3.3.5 Zusammenfassung . . . . . . . . . . . . . . . . . 54

3.4 Einfache Modelle-Resultate . . . . . . . . . . . . . . . 55

4 Modelle mit Gleichströmungsprofil $\quad 57$

4.1 Die Differentialgleichung für die kompressible Rohrströmung . . . 58

4.2 Wahl der Randbedingungen . . . . . . . . . . . . . . . . 58

4.3 Wahl des Grundströmungsprofils . . . . . . . . . . . . . . . 59

4.4 Zur Lösung der Differentialgleichung . . . . . . . . . . . . . . . 60

4.4.1 Die instabile Mode . . . . . . . . . . . . . . . 61

4.4 Die anderen Moden . . . . . . . . . . . . . . . . 64

4.5 Wechseldruckprofile ... . . . . . . . . . . . . . 64

4.6 Zusammenfassung . . . . . . . . . . . . . 66

5 Streuung $\quad \mathbf{6 8}$

5.1 Die WienER-Hopf-Methode . . . . . . . . . . . . . . 69

5.1.1 Der abstrakte Lösungsweg . . . . . . . . . . . . . . . . 69

5.2 Die Streurechnung nach KocH und MöHRING . . . . . . . . . . . 73

5.2.1 Wahl der Sprungbedingungen . . . . . . . . . . . . 74

5.2.2 Ergebnisse für den Rechteckkanal . . . . . . . . . . . 76

5.3 Rohrgeometrie. . . . . . . . . . . . . . . . 78

5.3.1 Ergebnisse für das Rohr . . . . . . . . . . . . 78

5.4 Zusammenfassung . . . . . . . . . . . . . . 83

6 Ergebnisse und Ausblick $\quad 85$

A Zur Ausbreitungsrichtung nach Briggs, Jones und Morgan $\mathbf{8 8}$

B Die Wiener-Hopf-Methode: Ein einfaches Beispiel 93

B.1 Lösungen mit elementaren Mitteln . . . . . . . . . . . . . . . . 93 
B.2 Demonstration der WienER-Hopf-Methode . . . . . . . . . . . . 95

$\begin{array}{lr}\text { C Zur Berechnung der Streufaktoren } & \mathbf{1 0 0}\end{array}$

C.1 Die dimensionslosen Grundgleichungen . . . . . . . . . . . . . . . 100

C.2 Aufstellen der WienER-Hopf-Gleichung . . . . . . . . . . . . . . 103

C.3 Zerlegen der Wiener-Hopf-Gleichung . . . . . . . . . . . . . . . 105

C.4 Sprungbedingungen . . . . . . . . . . . . . . . . . . . 109

C.5 Ausdrücke für die Streufaktoren . . . . . . . . . . . . . . . . . . . 113 


\section{Kapitel 1}

\section{Einleitung}

Zur Schalldämpfung in durchströmten Kanälen (etwa Einlaß- und Abgasleitungen von Verbrennungsmotoren; Klimaanlagen) benutzt man oft Wandauskleidungen mit Seitenkammern, deren Geometrie an die zu dämpfenden Frequenzen angepaßt sind. Häufig wird eine Kassettierung aus mehreren derartigen Kammern verwendet, so daß die Wand dann eine periodische Struktur besitzt.

Es ist seit langem bekannt, daß Auslegungen, die für fehlende oder geringe Grundströmung gute Dämpfungserfolge zeigen, bei höheren Strömungsgeschwindigkeiten versagen, siehe etwa MEchel [14]. Die Grundströmung stellt ein Reservoir dar, aus dem der Schall unter bestimmten Umständen Energie beziehen kann. Schallverstärkung ist daher möglich und tritt in der Praxis auch auf.

Es wäre daher wünschenswert, diese Effekte vorhersagen zu können, um Schalldämpfer von vornherein daran anpassen zu können.

Eine mathematisch wenig aufwendige Methode haben kürzlich AurÉGAN und LERoux [1] vorgeschlagen, bei der die Streumatrix des Gesamtobjektes aus den entsprechende Matrizen der Einzelsegmente zusammengesetzt wird. Die Reflexions- und Transmissionskoeffizienten des dazugehörigen Experimentes ließen sich reproduzieren, sofern Verluste des Axialimpulses in die Seitenkammern durch einen empirischen Faktor in den Erhaltungsgleichungen berücksichtigt wurden. Untersucht wurden aber nur Frequenzen unterhalb der Resonanz der Kammern.

Neben der Berechnung von Schallfeldern in periodisch ausgekleideten Kanälen stellt die sprunghafte Änderung der Wandeigenschaften am Einlauf in derartige Dämpfer - von schallhart zu schallnachgiebig im weitesten Sinne - und entsprechend am Auslauf ein großes Problem dar. Um dieser Frage nachzugehen, haben Koch und MöHRING in [10] das Modell eines Rechteckkanals untersucht, bei dem 
auf einer endlichen Länge zwei gegenüberliegender Wände lokal nachgiebig ausgekleidet sind. Sie fanden, daß die Streufaktoren eines solchen durchströmten Objektes stark davon abhängen, welchen Sprungbedingungen man für die unbekannten Felder an den beiden Übergängen fordert. Diese sind jedoch nicht ohne weiteres aus einfachen physikalischen Prinzipien ableitbar, da die Modellierung selbst höchst singulär ist - unstetige Veränderung der Wandimpedanz und ein RechteckGrundströmungsprofil - und diese Singularitäten an den Wandübergängen sogar zusammentreffen. Es ist daher nicht gesagt, daß die Lösung mit der größten Regularität an den Übergängen auch die physikalisch richtige ist.

Die Autoren schlugen daher sorgfältige Messungen der Streufaktoren vor, um diese Frage experimentell zu klären.

Um lokal nachgiebigen Wänden möglichst nahezukommen, wurden Objekte mit besonders schmaler Kassettierung entworfen, in denen feinmaschige Metallgaze mit geringem Strömungswiderstand die Kammern vom Kanalinneren trennt, um ein Hineingreifen der Grundströmung zu verhindern. Diese wurden in einem Kanal zur Bestimmung der Streufaktoren von turbulent durchströmten Einbauten vermessen, der in seinen Ursprüngen auf RONNEBERGER [20] zurückgeht und zuletzt von Brandes [2] und Enghardt [6] in Aufbau und Datenerfassung erweitert und modernisiert wurde.

KraUse [11] beobachtete bei besonders schmalen und tiefen Kammern, daß Schall bei Frequenzen oberhalb der Kammerresonanz verstärkt wird, wenn man ihn in Richtung einer Grundströmung einstrahlt. Dieses Phänomen, später in Abgrenzung zu einer anderen Erscheinung Typ I genannt, wurde von BRANDES in [3] weiter untersucht. Es wurde vorläufig als an der Wand laufende, räumlich angefachte, hydrodynamische Mode gedeutet, die am Stromauf-Ende der Kassettierung angeregt wird, im Meßobjekt der Grundströmung Energie entzieht und am Stromab-Ende zu einer Schallabstrahlung mit höherer Amplitude führt.

Gleichzeitig mit der Schallanfachung erhöht sich der Druckabfall im Kanalstück ganz beträchtlich, so daß man einen regelbaren, schnell ansprechenden Strömungswiderstand erhält, wenn man die Instabilität geeignet anregt. Da ein modulierter Druckgradient selbst Schall mit der Modulationsfrequenz abstrahlt, ließe sich auf diese Weise sogar - prinzipiell beliebig tieffrequenter - Schall ohne bewegliche Bauteile erzeugen. Das Prinzip einer solchen Steuerung wurde von LANGE im Rahmen einer Diplomarbeit [13] demonstriert.

Um die Natur der Instabilität aufzuklären und durch ein besseres Verständnis ihr Potential für Anwendungen auszuloten, wurden theoretische Untersuchungen notwendig. Darüber hinaus sollten Vergleiche theoretisch berechneter Streufaktoren mit den experimentellen Daten Rückschlüsse auf die in der Natur realisierten 
Sprungbedingungen an den Enden des Kanalstückes zulassen, sofern eine solche Wahl der Sprungbedingungen überhaupt möglich ist.

Mit dieser Arbeit soll ein Beitrag dazu geleistet werden. 


\section{Kapitel 2}

\section{Experiment}

In diesem Kapitel werde ich den experimentellen Befund erläutern, der uns zu diesen Untersuchungen veranlaßt hat.

Der Aufbau, mit dem die Daten gewonnen wurden, stammt von EnGHARDT und BRANDES und stellt eine Erweiterung und Vervollkommnung von Vorgängerexperimenten dar. Details lassen sich in den Diplomarbeiten [6] und [2] finden. Ich beschränke mich auf eine kurze Erläuterung des Prinzips.

Die Daten selbst wurden von BRANDES bereits in seiner Dissertation [3] vorgestellt und diskutiert. Ich werde die für die Instabilität Typ I wichtigsten Ergebnisse, insbesondere die Streufaktoren, hier noch einmal präsentieren, mit dem Ziel, sie mit meinen Modellrechnungen zu vergleichen.

\subsection{Aufbau und Meßprinzip}

\subsubsection{Der Kanal}

Die Meßstrecke, an der das Schallverstärkungsphänomen beobachtet wird, dient der Ermittlung von Wellenzahlen und Streufaktoren in luftdurchströmten Kanälen. Sie besteht im wesentlichen aus zwei je drei Meter langen innenpolierten Messingrohren von $50 \mathrm{~mm}$ Durchmesser, die über unterschiedliche Meßobjekte verbunden werden können. Über einen Einlauftrichter mit aufgesetztem Strömungsgleichrichter wird Luft durch die Meßstrecke angesaugt undabhängig vom Strömungswiderstand des Meßobjektes-auf MACH-Zahlen bis 0,35 beschleunigt; siehe dazu Abb. 2.1.

Die mit dem Kanaldurchmesser gebildete REYNOLDS-Zahl erreicht für die höchsten erreichbaren Strömungsgeschwindigkeiten Werte von über $3 \cdot 10^{5}$, so 


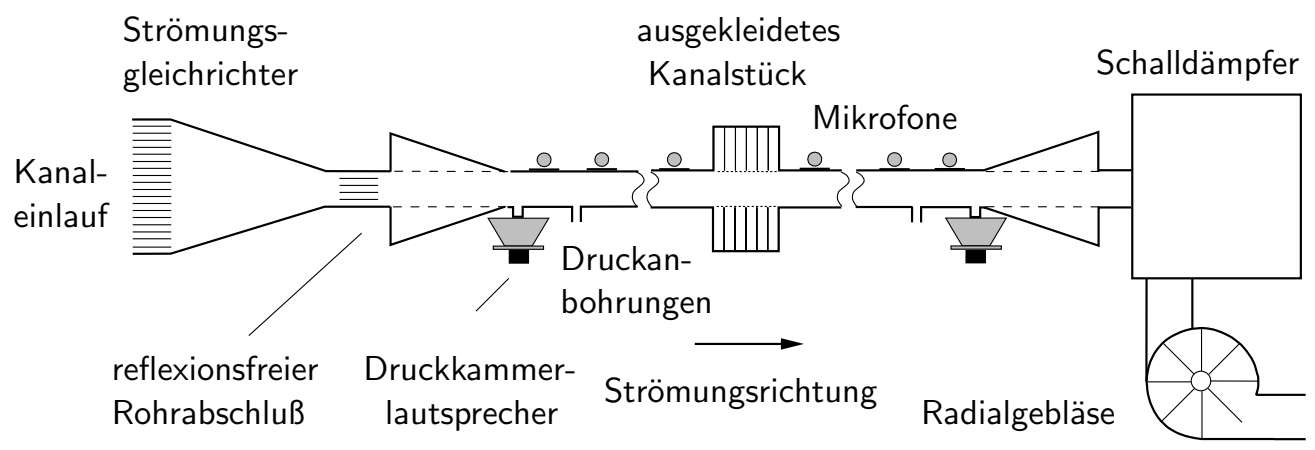

Abbildung 2.1: Aufbau des Strömungskanals zur Messung von Streufaktoren. An die Stelle des Kanalstückes können unterschiedliche Objekte eingebaut werden.

daß bereits bei Bruchteilen dieser Geschwindigkeiten am Ort des Meßobjektes (60 Rohrdurchmesser vom Einlauf entfernt) von einer voll entwickelten turbulenten Kanalströmung ausgegangen werden kann.

Die Enden des Kanals sind reflexionsarm abgeschlossen. Das Radialgebläse, welches die Luft ansaugt, ist von der Meßstrecke durch einen Schalldämpfer akustisch abgekoppelt. Wegen des verhältnismäßig geringen Durchmessers ist innerhalb der (schallharten) Teilrohre bis etwa $4 \mathrm{kHz}$ nur die Grundmode ausbreitungsfähig, wobei sich diese obere Grenze bei Zunahme der MACH-Zahl mit dem Faktor $\left(1-M a^{2}\right)^{1 / 2}$ noch etwas erniedrigt.

An den Rohr-Enden sind Druckkammerlautsprecher eingebaut, mit denen das Meßobjekt beschallt wird. Die Messung des Wechseldruckfeldes übernehmen 32 Mikrofone, welche durch Bohrungen mit dem Kanalinneren verbunden sind. Sie wurden so positioniert, daß bei Frequenzen bis hinab zu $30 \mathrm{~Hz}$ gemessen werden kann ${ }^{1}$.

Neben dem Schallfeld werden Luftfeuchte und Temperatur im Labor sowie die statischen Drücke am Ein- und am Auslauf der Meßstrecke gemessen, um die übrigen akustisch relevanten Strömungsparameter ermitteln zu können.

\subsubsection{Das Meßprinzip}

Das von BRANDES verwendete Meßverfahren geht in seinen Ursprüngen auf RoNNEBERGER [20] zurück. Ziel ist die möglichst genaue Bestimmung der Wellen-

\footnotetext{
${ }^{1}$ Die Abstände der Mikrofone werden mit zunehmender Entfernung vom Meßobjekt exponentiell größer.
} 


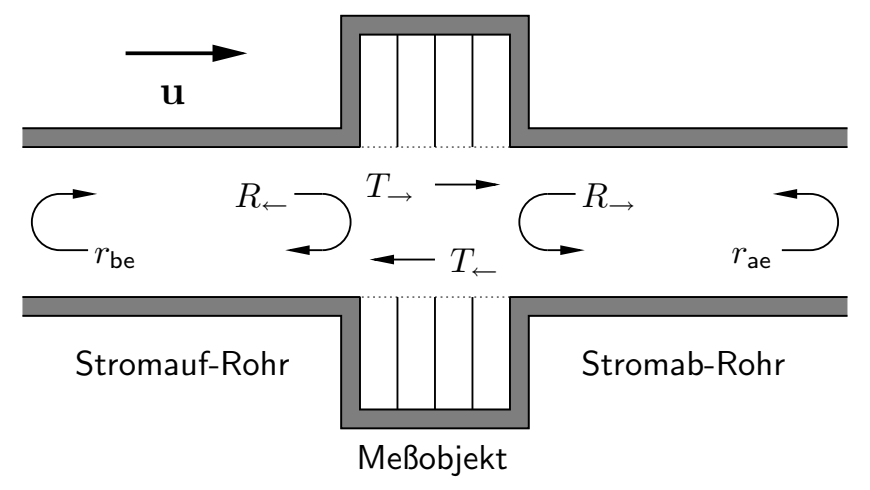

Abbildung 2.2: Bezeichnung der Streufaktoren

zahlen in den Meßrohren und der Streufaktoren an wie auch immer gearteten Einbauten im Kanal durch Abtastung des Wechseldruckfeldes in den Teilrohren. Die gesuchten Größen werden aus den Einzeldrücken mit Hilfe einer Ausgleichsrechnung gewonnen.

Da die Beschallungsfrequenz die cutoff-Frequenz der ersten höheren Mode nicht überschreitet, überlagern sich in den Teilrohren lediglich die Grundmode und ihre Echos an den Abschlüssen (Reflexionsfaktoren $r_{\mathrm{ae}}$ und $r_{\mathrm{be}}$ ) und den Übergängen ins Innere des Meßobjektes $\left(R_{\leftarrow}\right.$ bzw. $R_{\rightarrow}$; siehe Abb. 2.2). Darüber hinaus kommunizieren die Wellenfelder noch über die Transmissionsfaktoren $T_{\rightarrow}$ und $T_{\leftarrow}$. Kennt man die Wechseldrücke an hinreichend vielen Orten entlang der Meßstrecke, dann kann man die Wellenzahl und insbesondere alle Streufaktoren bestimmen.

Der experimentelle Aufwand und das Auswerteverfahren sind außerordentlich umfangreich, verglichen mit der Kürze, mit der sie hier geschildert wurden. Genauere Beschreibungen finden sich in den eingangs genannten Arbeiten.

\subsubsection{Die Meßobjekte}

Um die Schallstreuung an Kanalsegmenten mit lokal nachgiebigen Wänden untersuchen zu können, wurden bereits vor BRANDES Einbauten wie in Abb. 2.3 entworfen.

Es handelt sich im wesentlichen um eine Aneinanderreihung von ringförmigen Quasi- $\lambda / 4$-Resonatoren, die vom Kanal durch feinmaschige Metallgaze getrennt sind. Die Kammern sind am äußeren Umfang schallhart verschlossen und 


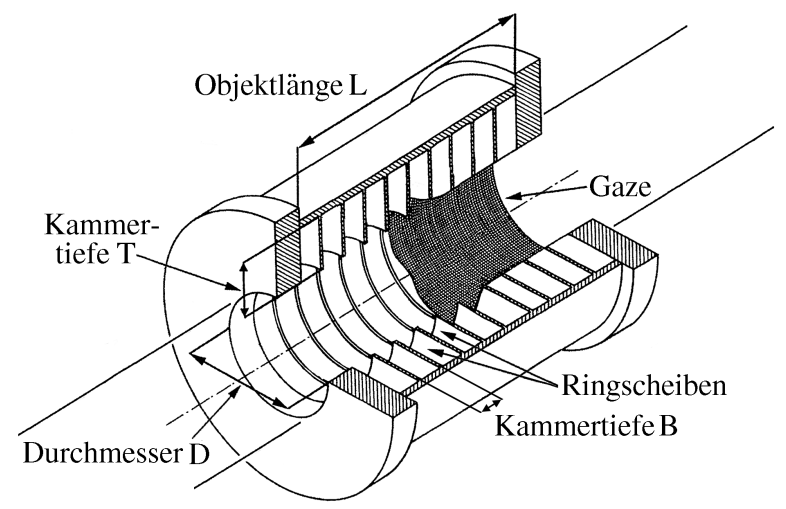

Abbildung 2.3: Schnitt durch ein Meßobjekt (nicht maßstäblich)

sorgfältig abgedichtet. Durch die verhältnismäßig große Tiefe und den geringen Abstand der Ringscheiben, deren Dicke 0,5 mm beträgt, wurde versucht, eine möglichst lokal reagierende Auskleidung zu erhalten. Der Strömungswiderstand der Gaze ist mit 0,04 $\rho c$ verhältnismäßig gering, wodurch der Wechseldruck dort fast verschwindet, wenn die Tiefenresonanz der Kammern erreicht wird. Die niedrigste Resonanzfrequenz liegt bei $f_{0}=849 \mathrm{~Hz}$, die erste Antiresonanz bei etwa $1700 \mathrm{~Hz}$, wobei sich die weitere Analyse nur auf Frequenzen bis zu dieser Antiresonanz beschränken wird. Für niedrige Frequenzen (unterhalb der Tiefenresonanz) gibt die Wand federnd, für höhere Frequenzen (bis zur Antiresonanz) masseartig nach; siehe auch Abschnitt 3.1.2.

Von BRANDES wurden insgesamt fünf verschiedene Objekte untersucht, wobei ich mich im folgenden meist auf dasjenige konzentrieren werde, bei dem die Verstärkung am ausgeprägtesten zu sehen war: Für dieses Objekt ist

- die Kammertiefe $T=75 \mathrm{~mm}$ (Resonanzfrequenz $f_{0}=849 \mathrm{~Hz}$ )

- die Kammerbreite $B=5 \mathrm{~mm}$,

- die Gesamtlänge ca. $L=87,5 \mathrm{~mm}$ (16 Kammern).

Obwohl die Hauptströmung von den Resonatorkammern durch die Gaze getrennt ist und in diesen keine merkliche (mittlere) Strömung vorhanden ist, wirbeln doch Turbulenzballen in die Kammern hinein und verlieren dort Impuls. Ich muß also davon ausgehen, daß sich innerhalb des Objektes an der Innenseite andere Wandschubspannungen und andere Geschwindigkeitsprofile ausbilden als im schallharten Rest des Kanals. 
Der Einfluß der soeben beschriebenen Auskleidung auf die Schallausbreitung läßt sich durch eine Wandimpedanz $Z$ (vorgeschriebenes Verhältnis von Wechseldruck und Schnelle senkrecht zur Wand) als Funktion der Frequenz beschreiben, wenn die Kammern schmal im Vergleich zu den relevanten Wellenlängen sind. $Z$ kann mit dem entsprechenden Eigenfunktionssystem (hier: BEssel- und NEUMANNFunktionen) berechnet werden. Ich komme darauf aber noch in Kapitel 3 zurück.

\subsection{Die Instabilität Typ I}

\subsubsection{Streufaktoren}

Ich präsentiere zunächst die von BRANDES experimentell ermittelten Wechseldruck-Streufaktoren für das im vorigen Abschnitt beschriebene Meßobjekt. Ich bezeichne mit $T_{\rightarrow}$ den Transmissionsfaktor, mit dem eine in Strömungsrichtung eingestrahlte Schallwelle das Objekt durchquert, mit $R_{\leftarrow}$ den dazugehörigen Reflexionsfaktor und mit $T_{\leftarrow}, R_{\rightarrow}$ die entsprechenden Größen bei Beschallung stromauf.

In Abb. 2.4 sind die Beträge der Streufaktoren bei Beschallung in Strömungsrichtung zu sehen. Während bei ruhender Luft das kassettierte Kanalstück wie erwartet Frequenzen nahe der Tiefenresonanz dämpft, ist bereits bei $M a=0,10$ dieser Dämpfungserfolg durch einen Verstärkungsbereich knapp oberhalb der Resonanz wieder aufgebraucht. Für noch höhere Strömungsgeschwindigkeiten erreicht der Betrag des Transmissionsfaktors sogar Werte über Eins, bis zu einer Verstärkung von ca. $30 \mathrm{~dB}$ bei $M a=0,24$.

Der Reflexionsfaktor und die entsprechenden Größen bei Beschallung entgegen der Strömung (siehe Abb. 2.5) zeigen eine weniger ausgeprägte MACHZahl-Abhängigkeit. Insbesondere gibt es keine Schallverstärkung bei Beschallung stromauf. Die Werte über Eins bei $R_{\leftarrow}$ resultieren aus der allgemeinen MACH-Zahl-Abhängigkeit des Reflexionsfaktors selbst und sind kein Ausdruck eines Verstärkungsphänomens.

In Abb. 2.6 ist die Phase des Transmissionsfaktors $T_{\rightarrow}$ für vier verschiedene Machzahlen dargestellt.

Man sieht, daß die Phasendrehung im Verstärkungsbereich stark zunimmt, um sich bei Frequenzen darüber nur noch moderat zu ändern.

Die in der genannten Arbeit vorhandenen Fehlerbalken habe ich in diesen Diagrammen der Übersicht halber weggelassen. 

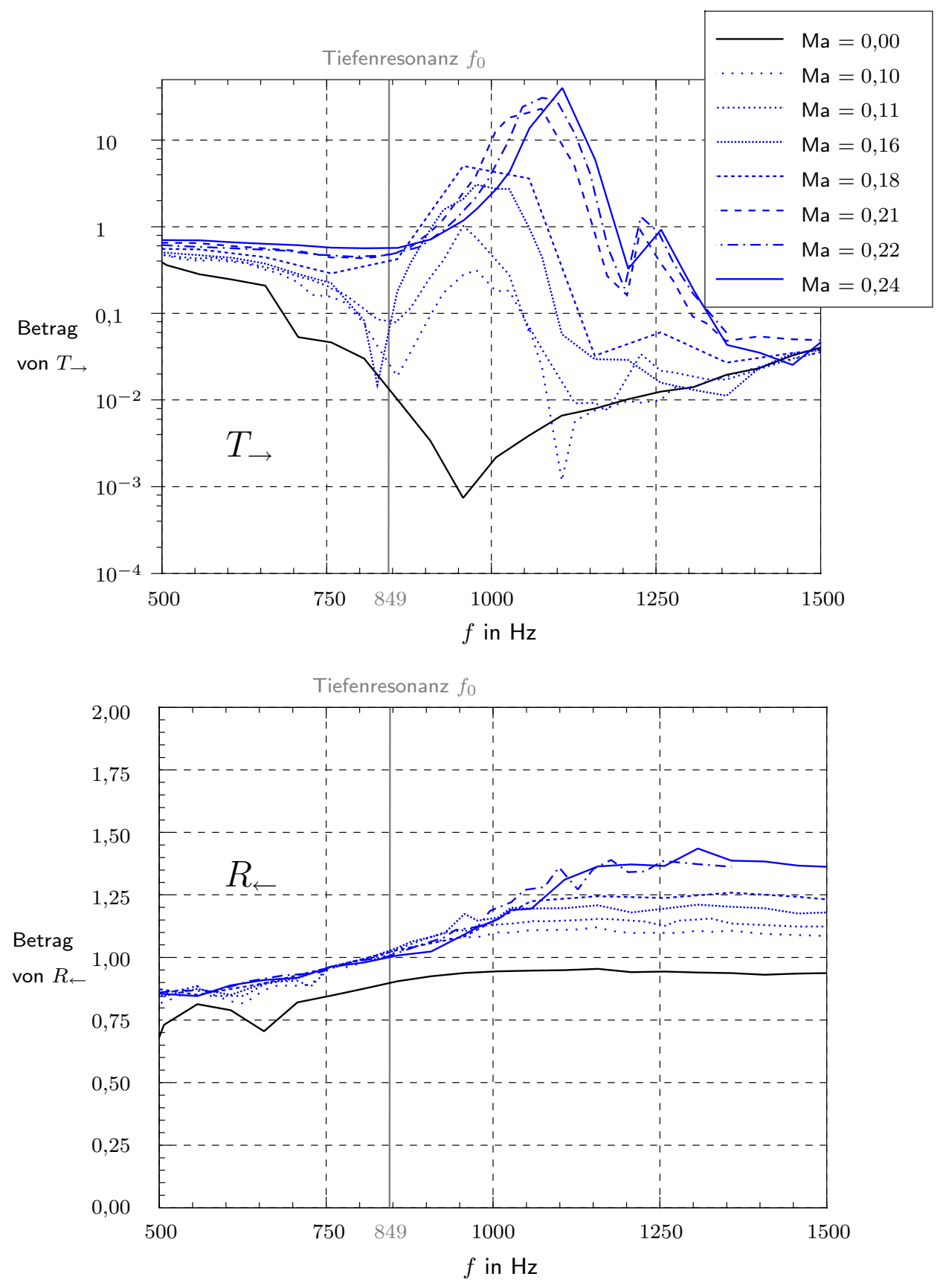

Abbildung 2.4: Beträge des Transmissions- und des Reflexionsfaktors bei Beschallung in Stromab-Richtung. Aus: Diss. BRANDES 


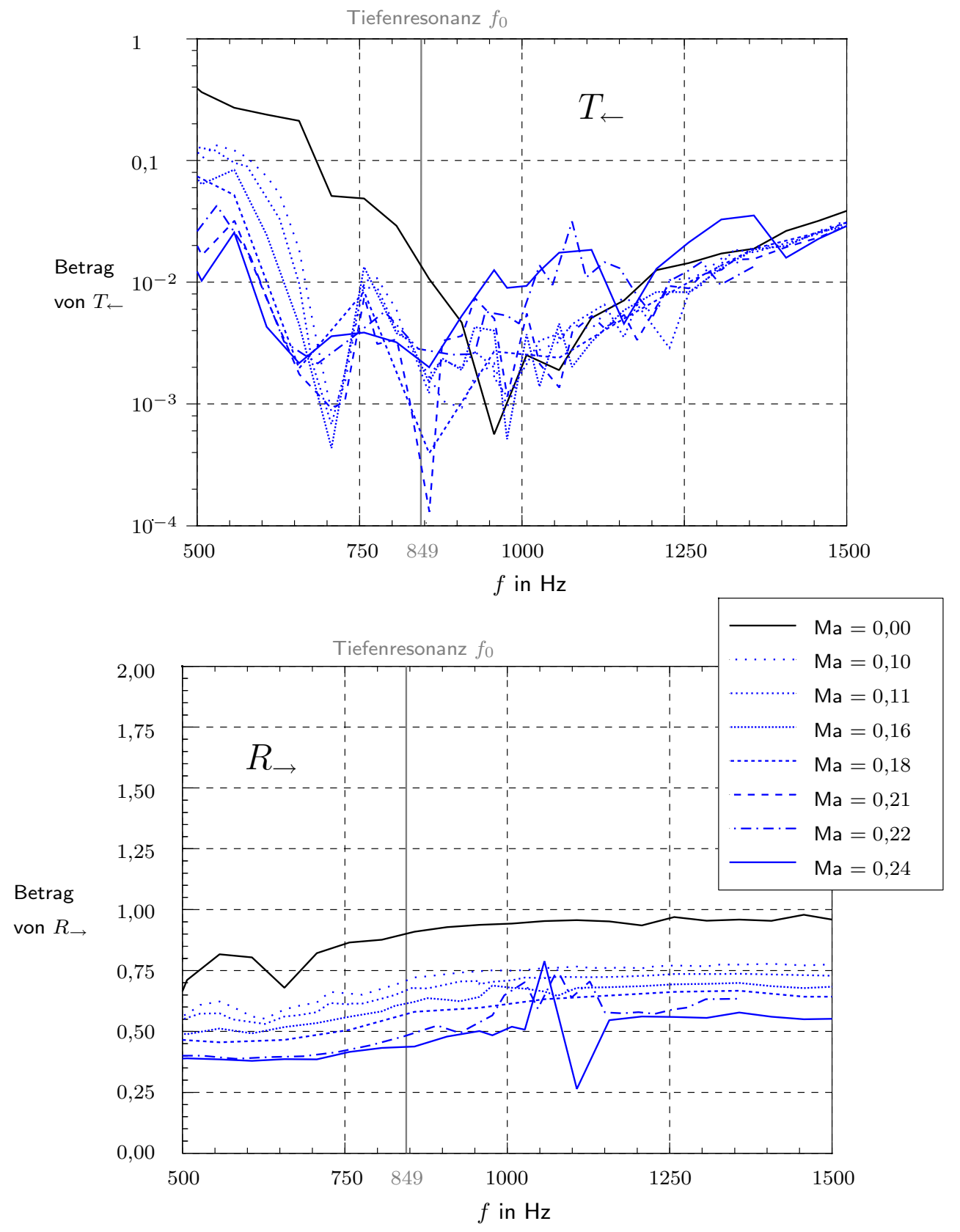

Abbildung 2.5: Beträge des Transmissions- und des Reflexionsfaktors bei Beschallung stromauf. Die Abweichungen von $R \rightarrow$ vom generellen Verlauf bei ca. $1100 \mathrm{~Hz}$ sind nicht signifikant (große Meßunsicherheit, siehe Diss. Brandes). 


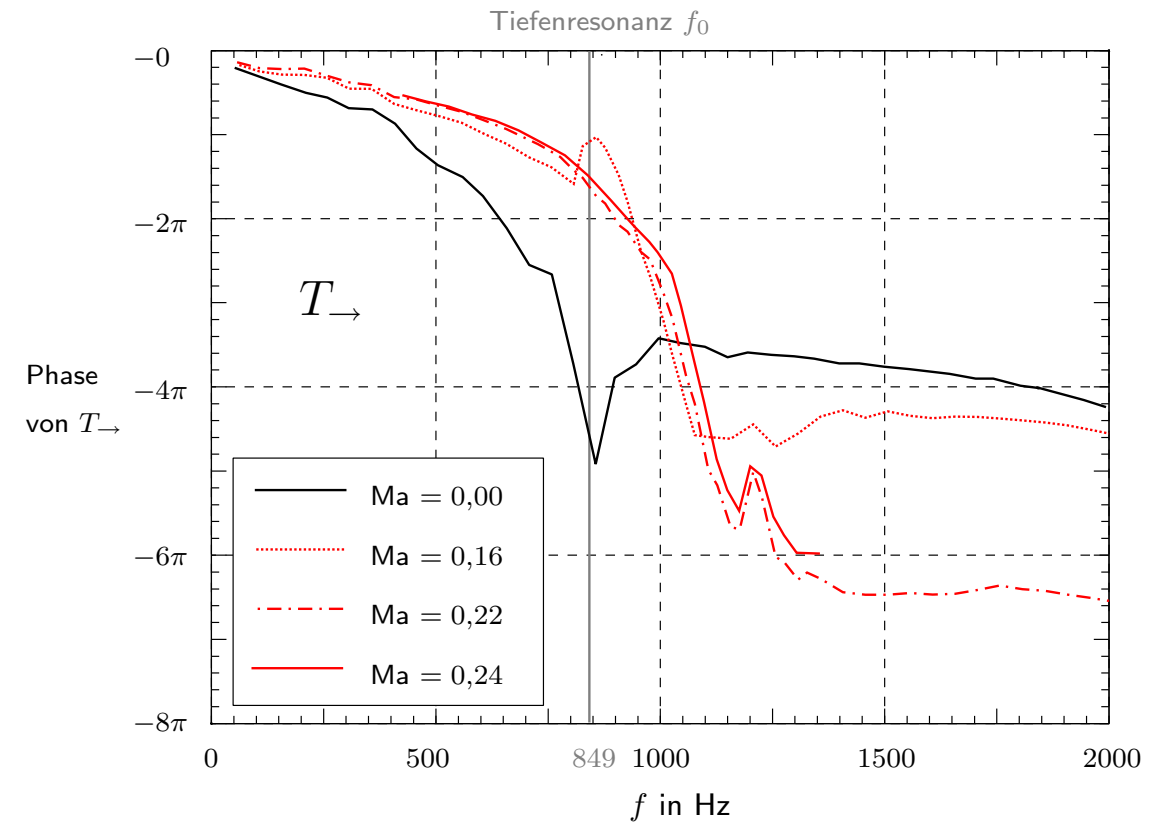

Abbildung 2.6: Phase des Transmissionsfaktors bei Beschallung stromab. Aus: Diss. BRANDES

\subsubsection{Abhängigkeit der Verstärkung von den experimentellen Para- metern}

Das Verhalten von $T \rightarrow$ läßt sich wie folgt zusammenfassen:

- Für große Strömungsgeschwindigkeiten gibt es ein Frequenzband mit einem erhöhten Transmissionsfaktor, wobei der Betrag über Eins steigen kann.

- Das Verstärkungsband erstreckt sich beginnend knapp oberhalb der Resonanzfrequenz bis zu einer darüber liegenden Frequenz, die für höhere MACHZahlen weiter ansteigt. Für das Kanalstück mit Resonanzfrequenz $849 \mathrm{~Hz}$ erreicht dieses bei MACH-Zahlen von 0,2 eine Breite von ca. $250 \mathrm{~Hz}$.

- Die Frequenz maximaler Verstärkung verschiebt sich mit wachsender MACHZahl ebenfalls nach oben.

- Die Maximalverstärkung steigt mit der MACH-Zahl und erreicht $30 \mathrm{~dB}$ bei $M a=0,24$. 


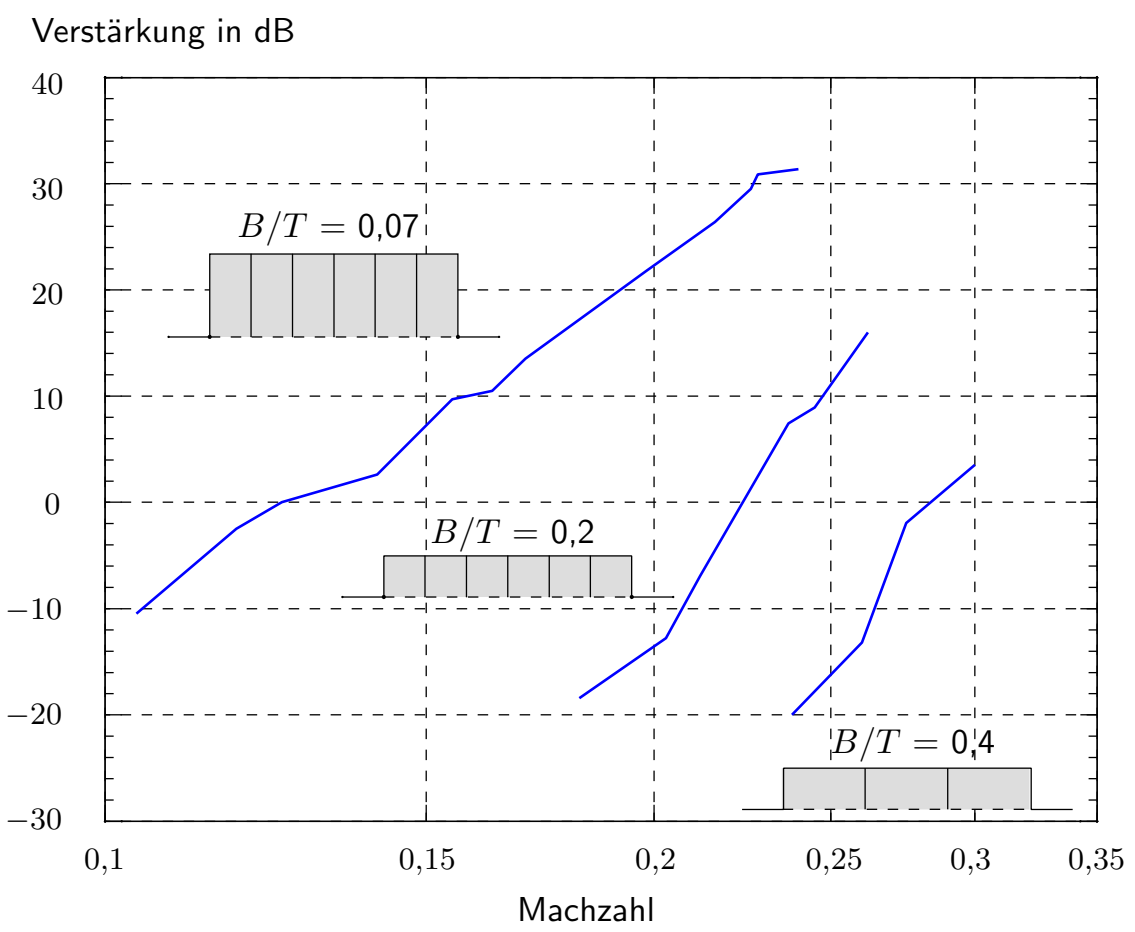

Abbildung 2.7: Maximalverstärkung in Abhängigkeit von der MACH-Zahl für verschiedene Kammergeometrien. Die Bauteillänge ist bei allen drei Kanalstücken gleich. Die Abbildungen sind nicht maßstäblich. Aus: Diss. Brandes

- Innerhalb des Verstärkungsbereiches ändert sich die Phase des Transmissionsfaktors mit der Frequenz viel stärker als außerhalb.

BRANDES untersuchte auch, wie die Verstärkung von den charakteristischen Abmessungen des ausgekleideten Kanalstückes abhängt. Die wesentlichen Beobachtungen lauten:

- Die Verstärkung ist um so höher, je schmaler die Kammern sind. In Abb. 2.7 wird die Maximalverstärkung dreier gleich langer Kanalstücke verglichen. Den größten Effekt erzielt die Auskleidung mit den tiefen Kammern (und entsprechend tiefer Resonanzfrequenz). Je größer die Kammerbreite $B$ im Verhältnis zur Tiefe $T$ wird, um so geringer ist die Verstärkung.

- Es besteht kein einfacher Zusammenhang zwischen Objektlänge $L$ und dem Verstärkungsfaktor (siehe Abb. 2.8). Zwischen acht und sechzehn Kammern 


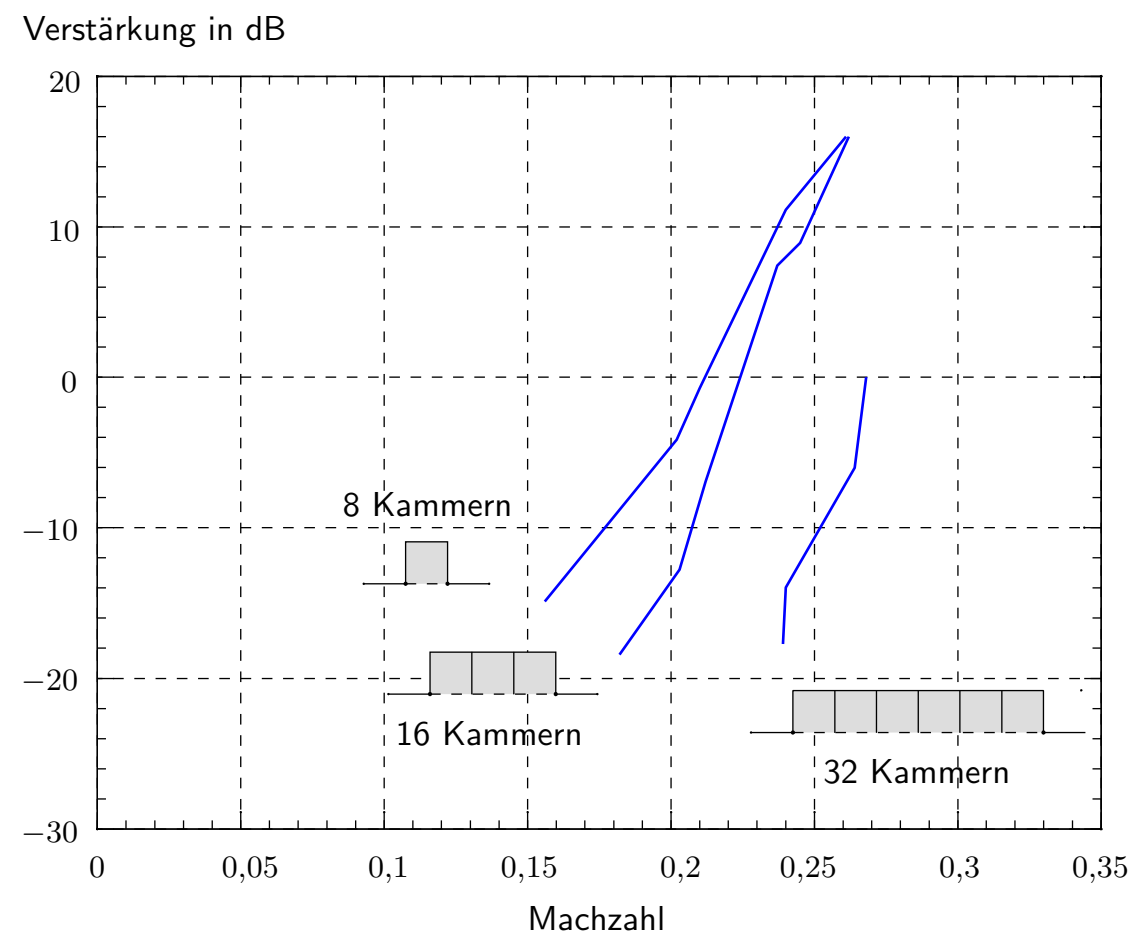

Abbildung 2.8: Maximalverstärkung in Abhängigkeit von der MACH-Zahl für verschiedene Bauteillängen. Aus: Diss. Brandes

besteht nur ein geringer Unterschied in der Wirksamkeit der Anfachung, für das lange Objekt mit 32 Kammern ist sie dagegen schon deutlich geringer.

BRANDEs hat noch einige andere Abhängigkeiten untersucht, die ich aus Platzgründen hier nicht wiedergeben werde.

\subsection{Vorläufige Interpretation}

Die im vorangegangenen Abschnitt beschriebenen Beobachtungen wurden folgendermaßen gedeutet:

Für die Schallverstärkung in Stromabrichtung, in [3] Typ I genannt, ist eine hydrodynamische Instabilitätsmode verantwortlich. Sie wird am Stromauf-Ende des ausgekleideten Kanalstückes durch Schall angeregt und strahlt, nachdem sie sich angefacht ausgebreitet hat, unter Umständen am Stromab-Ende Schall mit 
höherer Amplitude ab.

Eine solche konvektiv angefachte Mode bezieht die Energie, die sie für die Vergrößerung ihrer Amplitude benötigt, aus der umgebenden Gleichströmung. Abhängig von der Anfangsamplitude der Mode im Verhältnis zur Stärke der Grundströmung beeinflußt dieser Energieentzug das Gleichströmungsprofil, so daß größere Bauteillängen nicht zwangsläufig zu einer Erhöhung der Anfachung führen.

Da schmalere Kammern zu größeren Verstärkungen führen, bevorzugt diese Mode offenbar lokal nachgiebige Wände, so daß diese Lösung auch in entsprechend vereinfachten Modellen mit Wänden geeignet gewählter Impedanz auftreten sollte.

Für das Verschwinden der Instabilität bei hohen Frequenzen können mehrere Ursachen verantwortlich sein. Es ist möglich, daß sich dann bei entsprechend geringen Wellenlängen die endliche Kammerbreite und der Verlust der Lokalität der Wand-Nachgiebigkeit bemerkbar macht. Die Mode könnte mit geringerer Wellenlänge auch immer mehr in Wandnähe rücken, wo die Strömungsgeschwindigkeit schon deutlich geringer und demzufolge weniger Energie zur Anfachung vorhanden ist.

Um die genaue Natur der Instabilität zu klären, wurde bereits von BRANDES die Modenstruktur entsprechender Modelle untersucht ${ }^{2}$. Er bestätigte die schon aus anderen Quellen — etwa [18], [10]—bekannte Existenz einer hydrodynamischen Instabilitätsmode, deren Parameterabhängigkeiten allerdings nur wenige Gemeinsamkeiten mit dem Experiment zeigten. Brandes und Ronneberger [19] schlossen daraus, daß in einem guten Modell die sonst meist vernachlässigte turbulente, von der Welle modulierte Wandschubspannung berücksichtigt werden müsse, weil durch die Einbeziehung des turbulenten Impulsverlustes an der Wand auch in anderen Fällen die Diskrepanzen zum Experiment geschlossen werden konnten (siehe [17]).

Ich beginne daher in Kapitel 3 mit der Untersuchung möglichst einfacher Modelle und ihrer Lösungen anhand entsprechender Dispersionsbeziehungen, bevor ich mich in Kapitel 5 Streurechnungen zuwende.

\footnotetext{
${ }^{2}$ Diese unveröffentlichten Arbeiten wurden nicht weiterverwendet.
} 


\section{Kapitel 3}

\section{Einfache Modelle}

Um den Einfluß instabiler Moden auf die akustischen Eigenschaften nachgiebig ausgekleideter Kanalsegmente zu verstehen, untersuche ich zunächst das Modenspektrum, das heißt, ich berechne die möglichen komplexen Wellenzahlen in einem homogenen Kanal in Abhängigkeit von Frequenz und Gleichströmungsgeschwindigkeit.

Dabei stellt sich die Frage, wie genau die Verhältnisse innerhalb des ausgekleideten Kanals modelliert werden müssen, inwieweit also etwa Details des Gleichströmungsprofils oder der Verlustmechanismen Einfluß auf den Verlauf der Wellenzahl haben.

\subsection{Inkompressibel überströmte Wand; Rechteckprofil}

Ein nicht besonders gutes, dafür aber mathematisch übersichtliches Modell ist das einer inkompressibel überströmten, lokal nachgiebigen Wand, wobei die Gleichströmung einem Rechteckprofil folgt. Dieses Modell ist-abgesehen von seiner kompressiblen Variante ${ }^{1}$ - offenbar auch das einzige geschlossen lösbare. Weitere Verfeinerungen führen zu Gleichungen, die nur noch numerisch behandelt werden können.

Die Vereinfachungen lassen sich wie folgt motivieren:

Wir vermuten, daß eine angefachte hydrodynamische Welle, vergleichbar etwa den Kelvin-Helmholtz-Wellen in einer freien Scherschicht, für die Verstärkung verantwortlich ist. Diese Lösung soll durch das Wechselspiel zwischen der Strömung

\footnotetext{
${ }^{1}$ Auch in diesem Falle erhält man eine Gleichung 4. Grades, die man aber nur noch mit großem Aufwand geschlossen lösen kann.
} 
im Kanalinneren und der resonanzartigen Kanalauskleidung zustandekommen. Da das Verstärkungsphänomen deutlicher zutage tritt, wenn die Kammerung feiner ist, betrachte ich anstelle der Auskleidung eine Wand mit lokaler Nachgiebigkeit. Das bedeutet, daß die Schnelle an einem Wandpunkt auch nur vom Wechseldruck an genau diesem Punkt abhängt. Das ist für endlich breite Kammern natürlich nicht der Fall.

Zum zweiten gehe ich davon aus, daß eine durch die nachgiebige Wand verursachte Lösung auch in Wandnähe lokalisiert ist. Da die Geometrie des Kanals und die Krümmung der Wand nicht entscheidend sein sollten, müßte es in einer ersten Näherung möglich sein, statt im Inneren eines Rohres in einem Halbraum zu rechnen, der durch eine Ebene begrenzt wird. Weil darüber hinaus bei den Messungen nur rotationssymmetrische Schallfelder erzeugt wurden, kommt der Anfachungsmechanismus offenbar ohne die umfängliche Koordinate (Zylinderkoordinate $\phi$ ) aus. Ich kann also auf die spannweitige Koordinate z verzichten und zweidimensional rechnen.

Zum dritten sollte es gerechtfertigt sein, das Medium als inkompressibel anzunehmen. Die Lösungen, nach denen ich suche, werden hydrodynamische Moden sein. Die Kompressibilität des Mediums ist für deren Dynamik nur eine Störgröße und nicht, wie bei Schallwellen, die Ursache. Weil für sie $u$ und nicht $c$ die charakteristische Geschwindigkeit ist, stellt es auch kein Problem dar, daß in der inkompressiblen Näherung $c$ unendlich wird.

Charakteristika der Strömung, die sicherlich einen bedeutenden Einfluß auf den Verlauf der Moden haben, sind das Strömungsprofil und der Impulstransport (im vorliegenden Falle in der Hauptsache turbulent). Für ein erstes Verständnis sehe ich aber davon ab und ersetze das Profil durch eine Rechteckströmung und vernachlässige jegliche ortsabhängige turbulente oder zähe Impulstransportterme, weil ich auf diese Weise statt einer Differential- nur eine Dispersionsgleichung nach $k_{x}$ auflösen muß und ich im ersten Fall-im Gegensatz zum zweiten - keinerlei Kontrolle über Art und Zahl der möglichen Lösungen hätte.

\subsubsection{Die Grundgleichungen}

Für dieses Modell lege ich das Koordinatensystem wie folgt:

Die lokal nachgiebig gedachte Wand liege in der Ebene $y=0$, wobei die Werte $y>$ 0 im Inneren der Strömung angenommen werden. Die Kammern der Auskleidung nehmen den Bereich $-T<y<0$ ein. Die x-Koordinate falle mit der Richtung der Gleichströmung zusammen, also 


$$
\mathbf{u}(y)=u \mathbf{e}_{\mathbf{x}}, y>0
$$

Die z-Achse liegt quer zur Gleichströmung und parallel zur Wand („spannweitig"Koordinate), findet aber in diesem zweidimensionalen Modell keine weitere Verwendung.

Die Kontinuitätsgleichung für die Wechselgeschwindigkeit v hat dann die folgende Gestalt:

$$
\operatorname{div} \mathbf{v}=\partial_{x} v_{x}+\partial_{y} v_{y}=0
$$

Diese Beziehung folgt aus der Divergenzfreiheit der inkompressiblen Gesamtströmung $\mathbf{u}+\mathbf{v}$ und der stationären Grundströmung $\mathbf{u}$ (Linearität der Kontinuitätsgleichung).

Die EuLER-Gleichung für die Wechselgrößen

$$
\rho\left(\partial_{t}+u \partial_{x}\right) \mathbf{v}=-\nabla p
$$

geht aus der entsprechenden Gleichung für die Gesamtströmung hervor, wenn man den quadratischen Term $(\mathbf{v} \nabla) \mathbf{v}$ als klein vernachlässigt.

Der Ansatz

$$
p, \mathbf{v} \propto e^{-i \omega t+i \mathbf{k x}}
$$

führt schließlich auf das Gleichungssystem

$$
\begin{aligned}
k_{x}^{2}+k_{y}^{2} & =0 \\
\mathbf{k} p & =\rho\left(\omega-u k_{x}\right) \mathbf{v}=: \rho \omega^{\prime} \mathbf{v}, \text { insbesondere } \\
k_{y} p & =\rho \omega^{\prime} v_{y}
\end{aligned}
$$

$\omega^{\prime}$ ist die durch die Grundströmung DopPLER-verschobene Kreisfrequenz. Überall dort, wo (im Zeitbereich) wegen der Grundströmung statt $\partial_{t}$ die konvektive Ableitung $\partial_{t}+u \partial_{x}$ steht, muß im Frequenzbereich $\omega$ durch $\omega^{\prime}$ ersetzt werden.

\subsubsection{Modellierung der Wandimpedanz}

Als Randbedingung für das Modell nehme ich an, daß die Wand lokal nachgiebig ist. Das heißt, Wechseldruck und der zur Wand senkrechte Anteil der Schnelle sind proportional: 


$$
p=-Z^{\prime} v_{y}, y=+0
$$

In die Proportionalitätskonstante $Z^{\prime}$ gehen drei Anteile ein:

1. das Verhältnis zwischen Wechseldruck und Vertikalschnelle am kanalseitigen Ende $(y=-0)$ der Kammer,

2. der zusätzliche Widerstand durch die Gaze und

3. ein Faktor, mit dem dieses Verhältnis durch die unendlich dünn gedachte Schicht vermittelt wird, in der $\mathbf{u}$ von Null $(y=-0)$ auf $u(y=+0)$ anwächst. Diese Schicht wird Wirbelschicht genannt, weil sich hier die Wirbeligkeit rot $\mathbf{u}$ konzentriert, die sonst über das ganze Profil $\mathbf{u}(y)$ verteilt ist.

Das Verhältnis zwischen Wechseldruck und wandnormaler Schnelle, die sogenannte Wandimpedanz $Z$, berechnet sich für Kammern der Tiefe $T$ mit dem Ansatz einer hin- und rücklaufenden Welle in der Kammer

$$
p, \mathbf{v} \propto e^{-i \omega t}\left(\alpha e^{+i \frac{\omega}{c} y}+\beta e^{-i \frac{\omega}{c} y}\right)
$$

und der Forderung, daß die Außenwand der Kammern schallhart ist,

$$
v_{y}=0, y=-T
$$

$\mathrm{Zu}$

$$
\frac{p}{v_{y}}=i \rho c \cot \left(\frac{\omega}{c} T\right), y=-0
$$

Der Strömumgswiderstand der Gaze beträgt etwa 0,04 $\rho c$ und muß hinzuaddiert werden, so daß ich insgesamt erhalte:

$$
Z(\omega)=\rho c\left(0.04+i \cot \left(\frac{\omega}{c} T\right)\right)
$$

Um das ebene Modell mit dem experimentellen Aufbau von BRANDES vergleichbar zu machen, wähle ich die Kammertiefe $T$ so, daß als Resonanzfrequenz der Auskleidung

$$
f_{0}=\frac{c}{4 T}
$$

gerade $849 \mathrm{~Hz}$ wie beim Objekt mit tiefen Kammern herauskommt. 


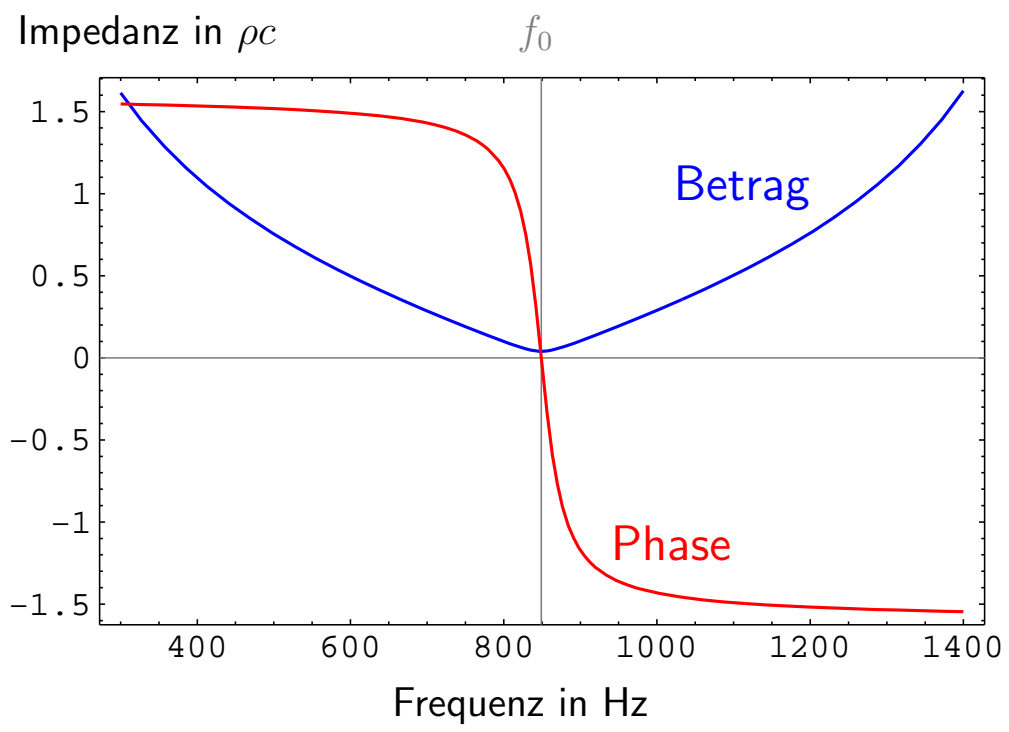

Abbildung 3.1: Betrag und Phase der Impedanz $Z / \rho c$ einer Wand, die lokal wie ein $\lambda / 4$-Resonator nachgibt. $T=101 \mathrm{~mm}$.

In Abb. 3.1 habe ich die Abhängigkeit der Wandimpedanz von der Schallfrequenz aufgetragen. Der Meßbereich umfaßt nur die Grund-Resonanzfrequenz (die nächsthöhere liegt bei $2547 \mathrm{~Hz}$ ) mit dem charakteristischen Wechsel der Phase von $+\pi / 2$ auf $-\pi / 2$ nahe $f_{0}$.

Um nun noch die Wirbelschicht zu berücksichtigen, in der die Gleichströmung vom Wert Null auf ihren vollen Betrag anwächst, führe ich die Auslenkung $\eta$ ein, die die wandnormale Versetzung kleiner Fluidelemente durch die Wechselströmung beschreibt. Es läßt sich zeigen, daß in einer ebenen Schichtenströmung sowohl der Wechseldruck als auch $\eta$ stetig durch die Wirbelschicht gehen:

$$
\begin{aligned}
& p(y=-0)=p(y=+0) \\
& \eta(y=-0)=\eta(y=+0)
\end{aligned}
$$

Da die zeitliche Ableitung $\partial_{t}$ und die konvektive Ableitung $\partial_{t}+u \partial_{x}$ vertauschen, folgt hieraus

$$
\begin{aligned}
\left(\partial_{t}+u \partial_{x}\right) \partial_{t} \eta(y=-0) & =\partial_{t}\left(\partial_{t}+u \partial_{x}\right) \eta(y=+0) \Rightarrow \\
\omega^{\prime} v_{y}(y=-0) & =\omega v_{y}(y=+0)
\end{aligned}
$$


wodurch ein zusätzlicher Faktor $\omega / \omega^{\prime}$ in der Randbedingung auftaucht,

$$
p=-\frac{\omega}{\omega^{\prime}} Z(\omega) v_{y}, y=+0
$$

In der Resonanz sind Wechseldruck und Schnelle an der Wand in Phase und der Wechseldruck ist so klein, daß bei dieser Frequenz die Schallausbreitung unterdrückt sein sollte. Beobachtet wird aber schon bei moderaten Machzahlen eine Verstärkung knapp oberhalb der Resonanz, wo die Nachgiebigkeit der Wand masseartig wird, das heißt, wo Druck und Strömung gegenphasig oszillieren.

\subsubsection{Lösung der Dispersionsbeziehung; Verlauf der Ausbreitungswel- lenzahlen}

Mit den Gleichungen (3.4) bis (3.6) und (3.8) kann ich die Moden, die sich über einer derartigen Wand ausbreiten können, explizit angeben. (3.4) löse ich nach $k_{y}$ auf, indem ich ein Vorzeichen einführe:

$$
k_{y}^{ \pm}= \pm i k_{x}^{ \pm}
$$

Mit dieser Wahl und (3.6), (3.8) erhalte ich schließlich folgende quadratische Dispersionsbeziehung für $k_{x}$,

$$
\pm i k_{x}^{ \pm} \omega Z=-\rho\left(\omega-u k_{x}^{ \pm}\right)^{2}
$$

mit den Lösungen

$$
k_{x}^{ \pm( \pm)}=\frac{\omega}{u}\left(1 \mp \frac{i Z}{2 \rho u}\left(1( \pm) \sqrt{1 \pm \frac{4 i \rho u}{Z}}\right)\right)
$$

RIEnstRA hat diese Wellenzahlen bereits in [18] angegeben. Bevor ich die Lösungen weiter untersuche, stelle ich ihren Verlauf $k_{x}(f)$ in Abbildung 3.2 für eine typische Strömungsgeschwindigkeit dar.

$\mathrm{Zu}$ diesen Kurven ist zu sagen, daß sie spätestens oberhalb derjenigen Frequenz irrelevant werden, für die der Realteil verschwindet, denn wegen Gleichung (3.9) verschwindet dort auch der Imaginärteil der Wellenzahl $k_{y}$. Von einer an der Wand lokalisierten Lösung kann dann keine Rede mehr sein, womit eine Voraussetzung für die (zumindest näherungsweise) Gültigkeit dieses Modells nicht mehr erfüllt ist. 

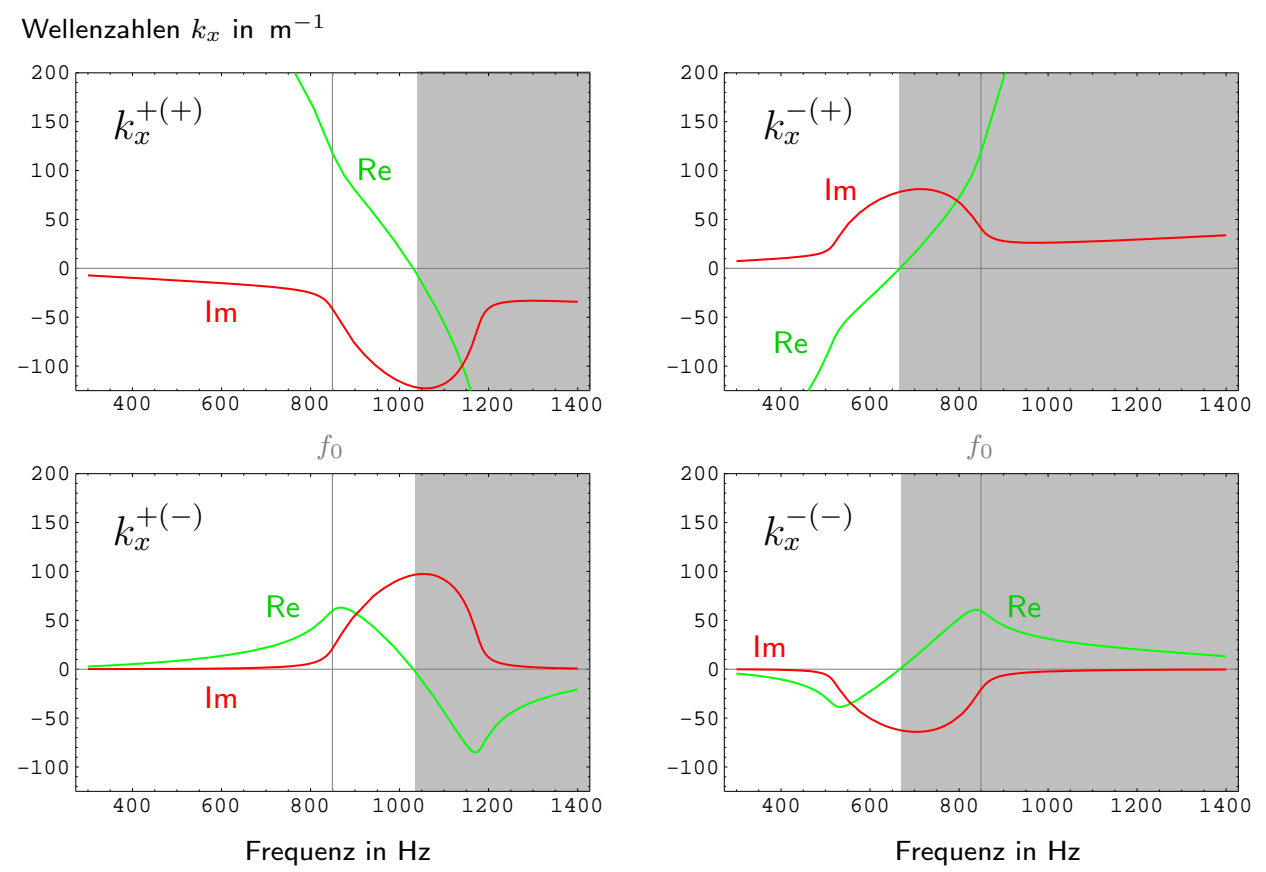

Abbildung 3.2: Die vier Lösungen $k_{x}^{ \pm( \pm)}$in Abhängigkeit von der Frequenz; Strömungsgeschwindigkeit $60 \mathrm{~m} \cdot \mathrm{s}^{-1}$. Grau unterlegt ist der Bereich, in dem eine Mode nicht mehr an der Wand lokalisiert ist $\left(\operatorname{Im} k_{y} \leq 0\right)$.

\subsubsection{Diskussion der Lösungen}

Ich untersuche zunächst, welche der vier Moden ohne Grundströmung auskommen. Dazu muß ich in (3.11) die dimensionslose Größe $\rho u / Z$ gegen Null gehen lassen. Ich kann für die Quadratwurzel eine Taylorentwicklung benutzen,

$$
\sqrt{1 \pm \frac{4 i \rho u}{Z}}=1 \pm \frac{2 i \rho u}{Z}+2\left(\frac{\rho u}{Z}\right)^{2}+\cdots
$$

Da die Wurzel bekanntlich nicht als stetige Funktion für alle komplexen Zahlen definiert werden kann, wähle ich als Schnitt in der komplexen Ebene den nicht-positiven Teil der reellen Achse, so daß für alle Argumente außerhalb dieses Strahls die Wurzel wohldefiniert ist. Insbesondere ist dann die gemachte Reihenentwicklung für den Radikanden (1+kleine Zahl) unkritisch und es haben alle Wurzeln wie üblich einen positiven Realteil.

Abhängig vom Vorzeichen $( \pm)$ erhalte ich die Entwicklungen 


$$
\begin{aligned}
k_{x}^{ \pm(+)} & =\frac{\omega}{u}\left(\mp \frac{i Z}{\rho u}+2+\cdots\right) \\
k_{x}^{ \pm(-)} & = \pm i \frac{\omega \rho}{Z}+\cdots
\end{aligned}
$$

in denen die Punkte Terme der Ordnung $\rho u / Z$ und höher vertreten.

Während das Paar $k_{x}^{ \pm(-)}$im Fall verschwindender Grundströmung in die generischen Oberflächenwellen einer nachgebenden Wand übergeht (löse (3.10) für $u=0$ ), sind die Lösungen $k_{x}^{ \pm(+)}$offenbar abwesend. Sie sind also hydrodynamische Moden, die bei fehlender Grundströmung nicht existieren können und von der Wechselwirkung des Strömungsprofils mit der ausgekleideten Wand erzeugt werden ${ }^{2}$.

Ebenso kann ich auch $Z$ gegen unendlich gehen lassen, also den Grenzfall schallharter Wände untersuchen. Mit einer identischen Reihenentwicklung ${ }^{3}$ erhalte ich das Resultat, daß das Paar hydrodynamischer Moden divergiert, bei dieser Randbedingung also gar nicht existieren kann, während die Wellenzahlen der Oberflächenwellen gegen Null gehen.

Ebenfalls interessant ist der Grenzfall verschwindender Frequenz. Für kleine $\omega$ ist $Z(\omega) \sim i \rho c^{2} /(\omega T)$, und man erhält entweder durch Reihenentwicklung der Wurzel (wie oben) oder direkt aus der Dispersionsbeziehung (3.10)

$$
\begin{aligned}
& k_{x}^{ \pm(+)}= \pm \frac{c^{2}}{u^{2} T} \\
& k_{x}^{ \pm(-)}=0
\end{aligned}
$$

Während die Oberflächenwellen aufhören zu existieren, werden die hydrodynamischen Lösungen im Grenzfall $\omega=0 \mathrm{zu}$ stationären, räumlich harmonischen Strömungen.

Als letztes untersuche ich die folgende Größe ${ }^{4}$ :

$$
v_{\mathrm{ph}} \doteq \frac{\omega}{|\operatorname{Re} \mathbf{k}|}=\frac{\omega}{\left|k_{x}\right|}
$$

\footnotetext{
${ }^{2}$ Analog verhalten sich die Kelvin-Helmholtz-Wellen in freien Scherschichten, die mit den gemachten Vereinfachungen hier von der Form $k_{\mathrm{KH}}=(\omega / u)(1 \pm i)$ wären.

${ }^{3} \rho u / Z$ ist klein, wenn $u$ klein oder $Z$ groß ist.

${ }^{4}$ Das zweite Gleichheitszeichen gilt nur im inkompressiblen zweidimensionalen Fall.
} 

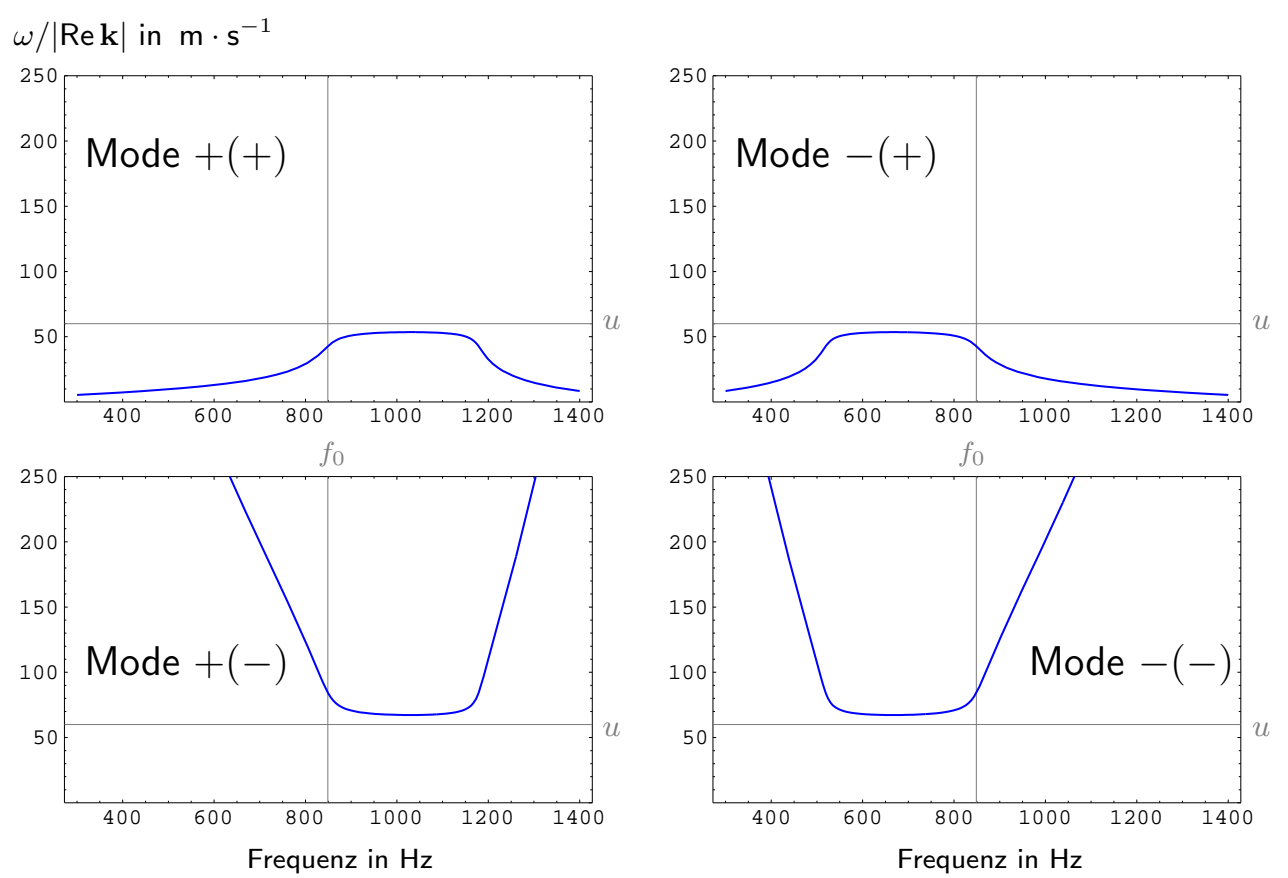

Abbildung 3.3: Betrag von $v_{\text {ph }}$ für die vier Lösungen bei einer Gleichströmung von $60 \mathrm{~m} \cdot \mathrm{s}^{-1}$

Anschaulich gesprochen ist dies die Geschwindigkeit, mit der sich die Fronten gleicher Phase ausbreiten, und zwar in Richtung des Lotes Rek (und nicht längs der Kanalachse). In Abb. 3.3 ist die Frequenzabhängigkeit von $v_{\mathrm{ph}}$ für die vier Lösungen zu sehen.

Es stellt sich heraus, das je zwei Moden in einem Frequenzband ober- oder unterhalb der Resonanzfrequenz annähernd dieselbe Frontengeschwindigkeit in der Größe der Grundströmungsgeschwindigkeit aufweisen. Außerhalb dieses Bereichs ist der Betrag für die hydrodynamischen Moden viel kleiner, für die Oberflächenmoden hingegen viel größer als der der Gleichströmung.

Dieses bemerkenswerte Verhalten darf allerdings nicht überinterpretiert werden, weil Real- und Imaginärteil der Wellenzahlen und demzufolge auch Frontenabstände und Anfachungslängen von derselben Größenordnung sind. In solchen Fällen ist nicht zu erwarten, daß Phasen- oder Gruppengeschwindigkeit etwas Zuverlässiges über die Ausbreitungsgeschwindigkeit einer Welle aussagen. 


\subsubsection{Die Ausbreitungsrichtung der Moden}

Ich will nun die Ausbreitungsrichtung der Wellen bestimmen. Für die generischen Moden $k_{x}^{ \pm(-)}$wäre es zunächst plausibel, diese aus dem Verhalten ohne Grundströmung abzuleiten: es existiert dann kein Energiereservoir, aus dem eine angefachte Mode gespeist werden kann, folglich sind die Lösungen bei $u=0$ in jedem Falle in Ausbreitungsrichtung gedämpft. $k_{x}^{+(-)}$sollte daher auch für $u>0$ stromab, $k_{x}^{-(-)}$hingegen stromauf laufen.

Voraussetzung für diesen Schluß ist jedoch, daß sich die Ausbreitungsrichtung bei Änderung der Strömungsgeschwindigkeit nirgendwo umkehrt. Für die hydrodynamischen Moden $k_{x}^{ \pm(+)}$ist die Ermittlung der Ausbreitungsrichtung auf diese Weise ohnehin nicht möglich, da sie ohne Grundströmung überhaupt nicht existieren.

Die Gruppengeschwindigkeit sagt ebensowenig Zuverlässiges über die Laufrichtung aus: Moden, deren Wellenzahlen einen großen imaginären Anteil besitzen, haben die Eigenschaft, daß ihre Wellenpakete sich schneller verformen als fortbewegen. Von einer klar erkennbaren Ausbreitungsrichtung kann deshalb keine Rede sein.

Das Problem der Ausbreitungsrichtung wurde unter anderem von BRIGGs [4] und Jones, Morgan [8] untersucht, wobei letztere die Verallgemeinerung eines Kriteriums von BRIGGS angeben. In beiden Fällen ergibt sich die Ausbreitungsrichtung aus der Forderung, daß die Lösung kausal sein soll, also für Zeiten vor dem Einschalten einer Quelle verschwindet:

Wenn man die Moden eines Randwertproblems mit Ausbreitungsrichtung $x$ untersucht, so liefert einem die Dispersionsbeziehung die Abhängigkeit zwischen $k_{x}$ und $\omega$, etwa aufgelöst nach $k_{x}=k_{x}(\omega)$. Wie kann man einer unendlich ausgedehnten Welle mit Wellenzahl $k_{x}$ ansehen, wohin sie sich ausbreitet? Es gibt weder eine örtlich begrenzte Quelle noch einen Beginn der Abstrahlung.

BRIGGS argumentiert nun, daß mit Übergang zu komplexen Kreisfrequenzen, wie sie einer zeitlichen Anfachung der Quelle entsprechen würden,

$$
\omega \mapsto \omega+i \sigma, \sigma>0
$$

alle Lösungen bei genügend hohem $\sigma$ schließlich in diejenige Richtung gedämpft sind, in die sie sich ausbreiten. Der Grund ist, anschaulich gesprochen, daß für genügend hohe Anfachungsraten das Anwachsen der Quelle eine Zunahme in Laufrichtung überkompensieren wird. Instabile Moden sollten sich also dadurch auszeichnen, daß sie bei großen $\sigma$ schließlich in der Richtung abklingen, in die sie 

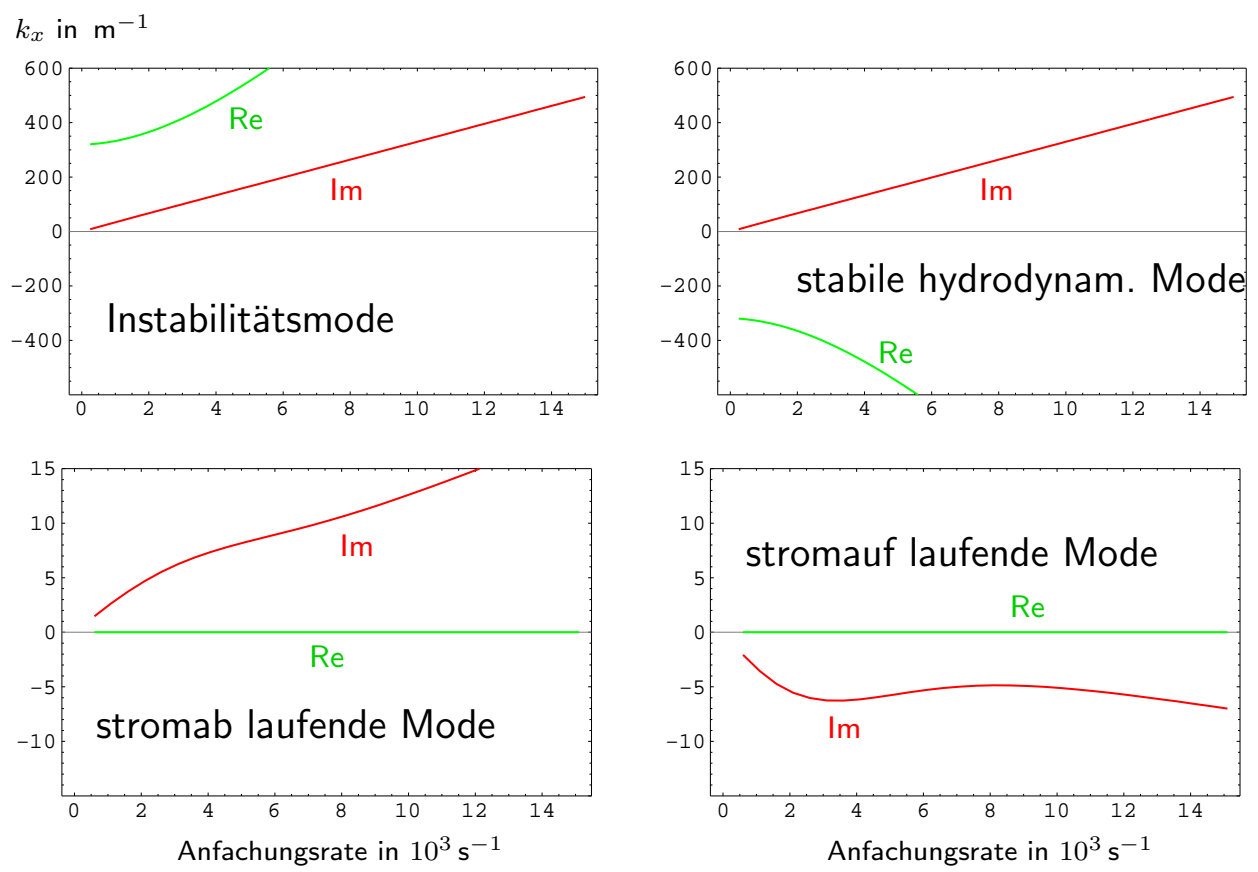

Abbildung 3.4: Wellenzahlen der vier Lösungen in Abhängigkeit von der Anfachungsrate $\operatorname{Im} \omega$ (kompressible Rechnung)

bei reellen Frequenzen anwachsen, für stromab laufende also:

$$
\operatorname{Im} k_{x}<0 \text { geht über in } \operatorname{Im} k_{x}>0
$$

Leider ist BRIGGS' Kriterium aus mathematischen Gründen, auf die ich in Anhang A eingehe, für dieses Problem nicht geeignet. Ich muß (wie RiEnstra [18]) die Verallgemeinerung von Jones und Morgan [8] benutzen, was im vorliegenden Fall darauf hinausläuft, den Imaginärteil der Wellenzahl wie eben beschrieben für rein imaginäre Frequenzen zu untersuchen.

Darüber hinaus ist es notwendig, kompressibel zu rechnen, was ebenfalls im Anhang erläutert wird.

Die Wellenzahlen für größer werdende, rein imaginäre Kreisfrequenz sind in Abb. 3.4 zu sehen: Es zeigt sich, daß das kompressible Analogon zu $k_{x}^{+(+)}$bei wachsender Anfachung in Grundströmungsrichtung gedämpft $\left(\operatorname{Im} k_{x}^{+(+)}>0\right)$ und daher auch bei reeller Frequenz eine stromab laufende, instabile Welle ist, während $k_{x}^{-(+)}$und $k_{x}^{+(-)}$gedämpft stromab laufen. Lediglich das kompressible Pendant 


\begin{tabular}{|c|c|c|c|c|c|}
\hline Mode & Typ & Richtung & Dämpfung & bei $u=0$ & bei $f=0$ \\
\hline$k_{x}^{+(+)}$ & hydrodyn. & stromab & angefacht & $\infty$ & $+\frac{c^{2}}{u^{2} T}$ \\
$k_{x}^{-(+)}$ & hydrodyn. & stromab & gedämpft & $\infty$ & $-\frac{c^{2}}{u^{2} T}$ \\
$k_{x}^{-(+)}$ & akustisch & stromab & gedämpft & $+\frac{i \omega \rho}{Z}$ & 0 \\
$k_{x}^{-(-)}$ & akustisch & stromauf & gedämpft & $-\frac{i \omega \rho}{Z}$ & 0 \\
\hline
\end{tabular}

Tabelle 3.1: Eigenschaften der vier Lösungen der inkompressiblen Wellengleichung vor einer resonanzartig nachgiebigen, ebenen Wand

zu $k_{x}^{-(-)}$klingt räumlich entgegen der Grundströmungsrichtung ab, folglich ist das die einzige Mode dieses Modells, die stromauf läuft.

Ich schließe diesen Abschnitt mit einer Übersicht über die gefundenen Moden in Tabelle 3.1.

\subsubsection{Zusammenfassung}

Es zeigt sich, daß vor nachgiebigen Wänden bei Strömungsüberlagerung zwei zusätzliche Moden existieren, die beide stromab laufen und von denen eine räumlich angefacht ist. Sie existieren nur vor dem Hintergrund einer Gleichströmung und bedürfen nachgiebiger Wände.

Die instabile hydrodynamische Mode könnte die Ursache der experimentell beobachteten Schallanfachung in Grundströmungsrichtung sein. Ein weiteres Indiz ist die Tatsache, daß sich mit dem Wechsel der Wandimpedanz von federartiger zu masseartiger Reaktion (bei der Resonanzfrequenz $f_{0}$ ) die Anfachung stark erhöht (siehe dazu Abb. 3.5), in Übereinstimmung mit der Beobachtung, daß die Schallverstärkung erst oberhalb von $f_{0}$ einsetzt. Eine obere Begrenzung des Verstärkungsbereiches existiert nicht, aber ein Rückgang des Anfachungsfaktors auf etwas niederigere Werte bei einer gewissen Frequenz oberhalb der Tiefenresonanz. Der besonders stark angefachte Bereich verbreitert sich mit wachsender Strömunsgeschwindigkeit, jedoch nimmt der Maximalwert der Anfachung, wie in Abb. 3.5 zu sehen, in selbem Maße ab, was der Transmissionskoeffizient stromab nach den Ergebnissen von BRANDEs gerade nicht tut. Das Verhalten der instabilen Lösung ist allerdings für hohe Frequenzen irrelevant, weil die Mode dort 


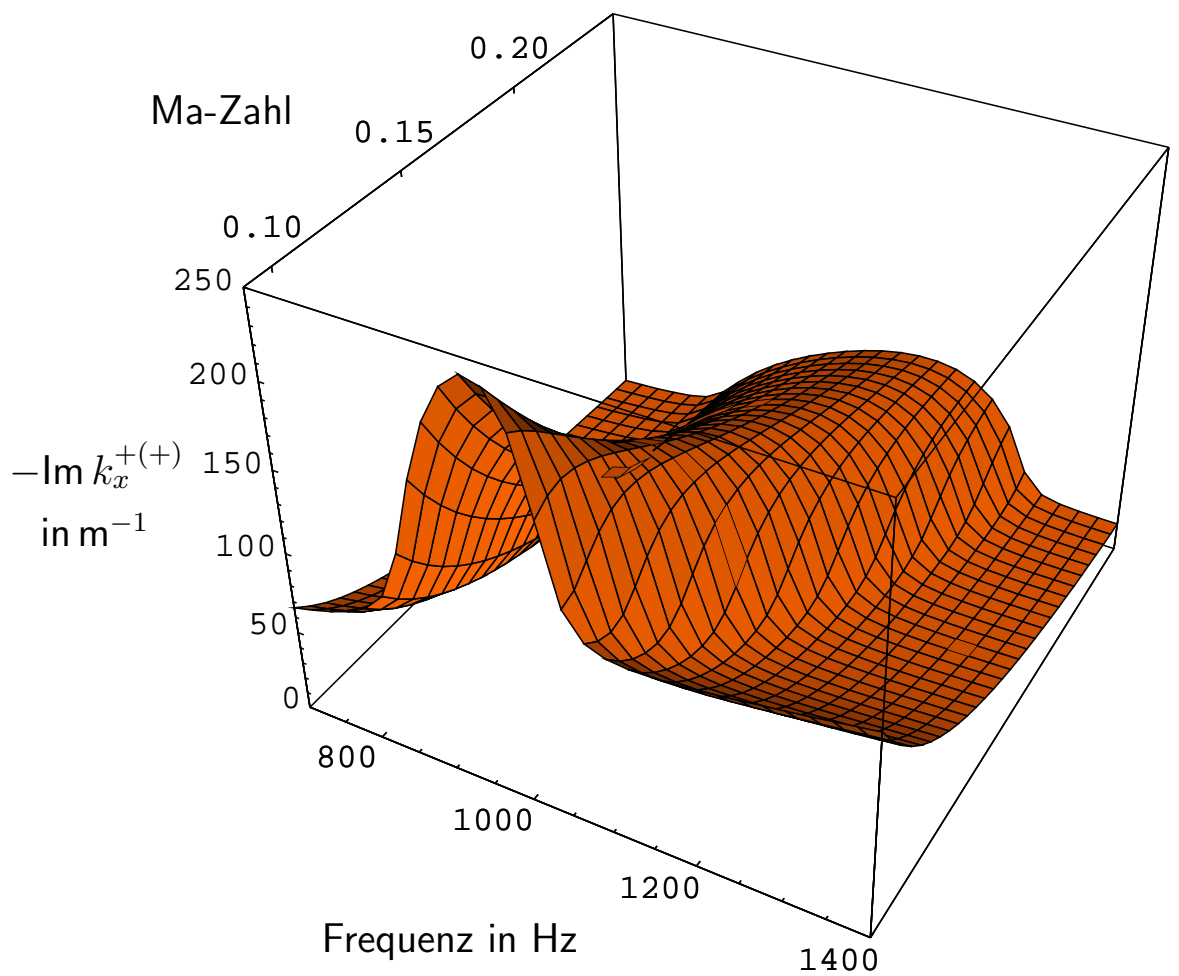

Abbildung 3.5: Der räumliche Anfachungsfaktor der Instabilitätsmode in Abhängigkeit von Frequenz und MACH-Zahl

bereits von der Wand weg angefacht ist und nichts mehr mit der tatsächlichen Lösung in einem endlich weiten Kanalstück zu tun hat. Die Untersuchung realistischer Geometrien ist daher zwingend erforderlich.

Für $60 \mathrm{~m} \cdot \mathrm{s}^{-1}$ liegt das Minimum im Imaginärteil bei etwa $125 \mathrm{~m}^{-1}$, was auf der Länge des ausgekleideten Objektes von 87,5 mm einer Anfachung von

$$
\exp (125 \cdot 0,0875)>5 \cdot 10^{4}
$$

entspräche. Das ist um Größenordnungen über der beobachteten Verstärkung. Verläßliche Aussagen über Transmissionsfaktoren kann aber nur eine Streurechnung liefern. 


\subsection{Kompressibilität; Andere Geometrien}

Im vorhergehenden Abschnitt habe ich die Instabilitätswelle an einer inkompressibel überströmten, ebenen Wand vorgestellt, die ein Kandidat für das von BRANDES und anderen untersuchte Verstärkungsphänomen Typ I ist. Ich untersuche nun, wie sich die Dispersionsbeziehung verhält, wenn man realistischere Modelle verwendet. Es ist dann zwar nicht mehr möglich, Lösungen explizit anzugeben, aber ich kann die Ergebnisse aus 3.1 als Richtschnur und Vergleich für die nur noch numerisch auffindbaren Lösungen benutzen.

\subsubsection{Effekte der Kompressibilität}

Zunächst interessiert mich der Einfluß, den die Kompressibilität der Luft auf den Verlauf der Lösungen hat.

Während sich die EulER-Gleichung (3.2) nicht ändert, hat die Kontinuitätsgleichung (3.1) nun die Form

$$
\partial_{t} \rho+\mathbf{u} \nabla \rho+\rho_{\mathbf{0}} \operatorname{div} \mathbf{v}=\mathbf{0}
$$

Sie ergibt sich aus der Kontinuitätsgleichung für die Gesamtströmung $\rho_{0}+$ $\rho, \mathbf{u}+\mathbf{v}$ bei Vernachlässigung der Terme, die quadratisch in den Wechselgrößen $\rho, \mathbf{v}$ sind. Als Zustandsgleichung, die die Beziehung zwischen Druck- und Dichteänderungen herstellt, benutze ich

$$
d p=c^{2} d \rho
$$

wobei die Schallgeschwindigkeit in allen Rechnungen den für Laborbedingungen angemessenen Wert von $343 \mathrm{~m} \cdot \mathrm{s}^{-1}$ haben wird.

Mit diesen Gleichungen und demselben Ansatz wie im inkompressiblen Fall wird aus (3.4)

$$
k_{x}^{2}+k_{y}^{2}-\left(\frac{\omega-u k_{x}}{c}\right)^{2}=0
$$

und aus (3.10) geht die Dispersionsbeziehung

$$
\pm i \sqrt{k_{x}^{2}-\left(\frac{\omega-u k_{x}}{c}\right)^{2}} \omega Z=-\rho\left(\omega-u k_{x}\right)^{2}
$$

hervor. 

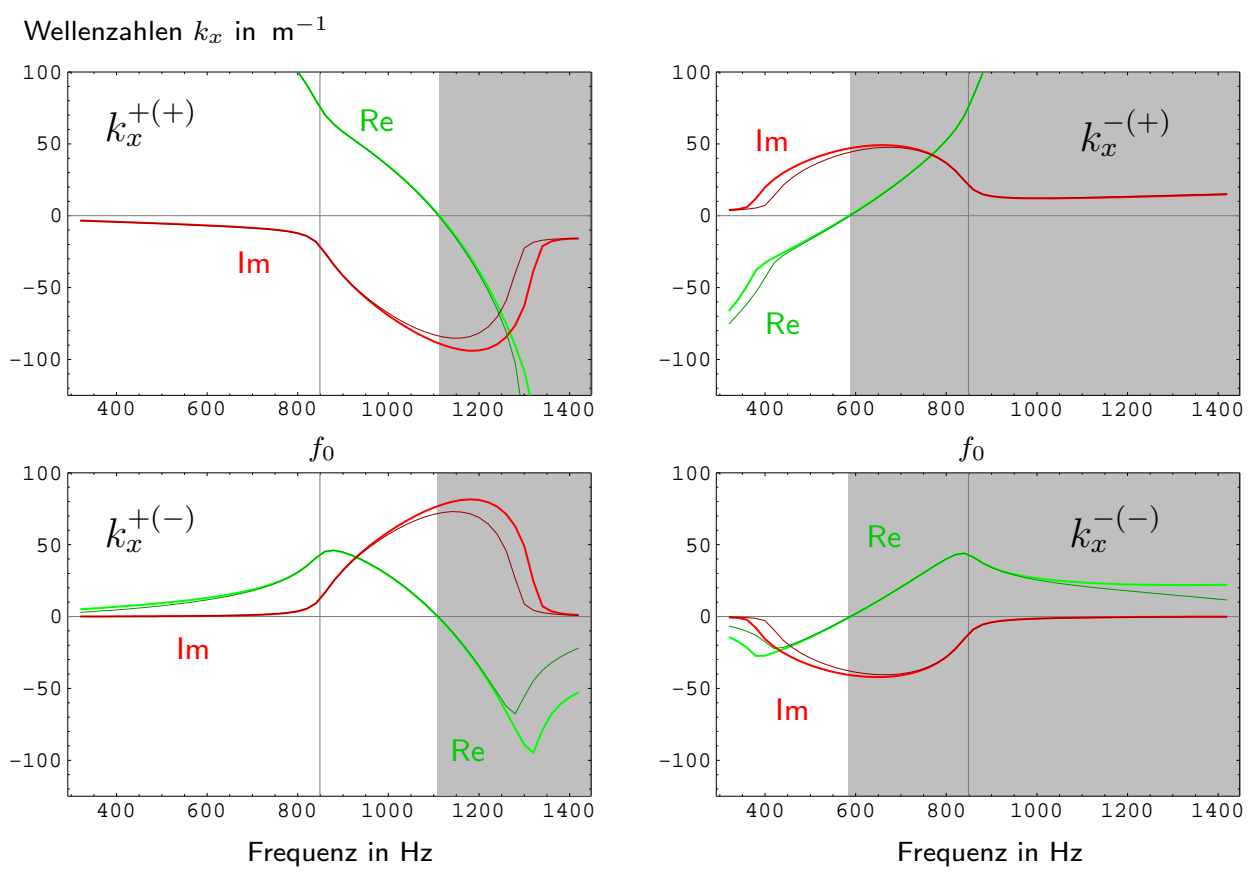

Abbildung 3.6: Vergleich der kompressiblen (dicke Linien) und inkompressiblen Lösungen (dünne Linien) bei $u=90 \mathrm{~m} \cdot \mathrm{s}^{-1}$

Die vier Lösungen habe ich-wie auch in den folgenden Modellen-mit der Mathematica-Routine FindRoot ermittelt.

Um eine Abschätzung für den Fehler zu erhalten, den eine inkompressible Rechnung mit sich bringt, vergleiche ich in Abbildung 3.6 die vier inkompressiblen Lösungen mit den kompressiblen und wähle dafür eine verhältnismäßig große Strömungsgeschwindigkeit von $90 \mathrm{~m} \cdot \mathrm{s}^{-1}(M a=0,26)$. Wie man sieht, sind die Abweichungen für diese (und damit auch kleinere) Strömungsgeschwindigkeiten und für Frequenzen in dem mich interessierenden Bereich moderat, so daß für ein erstes Verständnis ohne weiteres inkompressibel genähert werden kann. Allerdings vereinfachen sich gerade bei komplizierteren Modellen die Gleichungen auch nur unwesentlich, weswegen ich auf diese Vereinfachung oft verzichten werde.

Für komplexe Frequenzen zur Bestimmung der Ausbreitungsrichtung ergibt sich allerdings ein signifikanter Unterschied, der im Anhang A diskutiert wird. 


\subsubsection{Kanal mit kreisförmigem Querschnitt}

Ich untersuche nun die Wellenlösungen in einem Rohr mit dem Innendurchmesser der Meßobjekte, $D=50 \mathrm{~mm}$.

Ich bezeichne mit $x$ die Ortskoordinate in Ausbreitungsrichtung, mit $r$ die radiale Koordinate (die lokal nachgiebig gedachte Rohrwand liegt dann bei $r=R$ ) und die umfängliche Winkelkoordinate mit $\phi$. Im Vergleich zur ebenen Wand tritt $r$ dabei an die Stelle von $y, \phi$ entspricht der nicht benutzten Koordinate $z$.

Wie sich die EulER- und die (inkompressible) Kontinuitätsgleichung in Zylinderkoordinaten schreiben, läßt sich in Lehrbüchern nachschlagen, zum Beispiel [12]. Die resultierenden Gleichungen für die Wechselgrößen $p, \mathbf{v}=\left(v_{x}, v_{r}, v_{\phi}\right)$ bei Grundströmung in $x$-Richtung lauten:

$$
\begin{aligned}
\frac{1}{r} \partial_{r} r \partial_{r} v_{r}+\frac{1}{r} \partial_{\phi} v_{\phi}+\partial_{x} v_{x} & =0 \\
\left(\partial_{t}+u \partial_{x}\right) v_{x} & =-\frac{1}{\rho} \partial_{x} p \\
\left(\partial_{t}+u \partial_{x}\right) v_{r} & =-\frac{1}{\rho} \partial_{r} p \\
\left(\partial_{t}+u \partial_{x}\right) v_{\phi} & =-\frac{1}{\rho r} \partial_{\phi} p
\end{aligned}
$$

Hieraus läßt sich die inkompressible Wellengleichung (LAPLACE-Gleichung) in Zylinderkoordinaten herleiten:

$$
\Delta p=\left(\frac{1}{r} \partial_{r} r \partial_{r}+\frac{1}{r^{2}} \partial_{\phi}^{2}+\partial_{x}^{2}\right) p=0
$$

Der Ansatz für eine Schallmode im Rohrkanal lautet

$$
p \propto J_{m}\left(k_{r} r\right) e^{i m \phi} e^{-i \omega t+i k_{x} x}
$$

weil die Bessel-Funktionen $J_{m}$ Eigenfunktionen des LAPLACE-Operators in Zylinderkoordinaten sind. Die Wellengleichung reduziert sich zu

$$
k_{x}^{2}+k_{r}^{2}=0
$$

wenn man den Ansatz (3.25) in (3.24) einsetzt. 
Die Randbedingung bei $r=R$ lautet jetzt ${ }^{5}$

$$
v_{r}=\frac{\omega^{\prime}}{\omega} \frac{p}{Z(\omega)}
$$

und wird unter Benutzung der Gleichungen (3.22) und (3.25) zu der Dispersionsbeziehung

$$
k_{r} R \cdot J_{m-1}\left(k_{r} R\right)=\left(m+i\left(\omega-u k_{x}\right)^{2} \frac{\rho R}{\omega Z(\omega)}\right) J_{m}\left(k_{r} R\right)
$$

Dabei habe ich von der Beziehung Gebrauch gemacht, welche die Ableitung einer BESSEL-Funktion durch BESSEL-Funktionen ausdrückt.

In Gleichung (3.27) muß $k_{r}$ durch $k_{x}$ ausgedrückt werden, im inkompressiblen Fall, siehe (3.26),

$$
k_{r}= \pm i k_{x}
$$

Bei einer kompressiblen Rechnung ist entsprechend

$$
k_{r}= \pm i \sqrt{k_{x}^{2}-\left(\frac{\omega-u k_{x}}{c}\right)^{2}}
$$

einzusetzen. Das Vorzeichen ist irrelevant, weil es aus Symmetriegründen in (3.27) herausfällt.

Als Wandimpedanz wähle ich dasjenige Verhältnis von $p$ und $v_{r}$, das sich gerade bei der Kammergeometrie der experimentell verwendeten Meßobjekte ergibt. Die entsprechende Formel findet sich in [3] im Abschnitt 2.3 und läßt sich auf ähnliche Weise wie (3.7) herleiten. Sie involviert wegen der Zylindergeometrie jetzt Bessel- und NeUmanN-Funktionen,

$$
Z(\omega, m=0)=\rho c\left(0,04-i \frac{N_{1}\left(\frac{\omega}{c} R_{a}\right) J_{0}\left(\frac{\omega}{c} R\right)-J_{1}\left(\frac{\omega}{c} R_{a}\right) N_{0}\left(\frac{\omega}{c} R\right)}{N_{1}\left(\frac{\omega}{c} R_{a}\right) J_{1}\left(\frac{\omega}{c} R\right)-J_{1}\left(\frac{\omega}{c} R_{a}\right) N_{1}\left(\frac{\omega}{c} R\right)}\right)
$$

Der Außenradius der Kammern beträgt im Falle der Resonanzfrequenz $849 \mathrm{~Hz}$ $R_{a}=100 \mathrm{~mm}$.

Diese Impedanz gilt so nur für den Fall $\phi$-unabhängiger Wechselfelder. Für Lösungen mit $m \neq 0$ ist die Auskleidung aus dem Experiment nicht mehr lokal nachgiebig, weil eine Querunterteilung in umfänglicher Richtung fehlt.

\footnotetext{
${ }^{5}$ Weil ein positives $v_{r}$ in die Wand hinein zeigt, erscheint im Gegensatz zur Rechnung an der ebenen Wand kein Minuszeichen.
} 
Die Dispersionsbeziehung (3.27) hat unendlich viele Lösungen. In den einfachen Hohlleiterproblemen ohne Strömung und mit schallharten Wänden gibt es, abhängig von der Frequenz, typischerweise einige ausbreitungsfähige Moden mit reeller Wellenzahl $k_{x}$ und unendlich viele höhere imaginäre Moden, die evaneszent sind. In der inkompressiblen Näherung sind alle Moden rein imaginär.

Als Lösungen erwartet man demnach unendlich viele $k_{x}$ in der Nähe der imaginären Achse und wegen der nicht verschwindenden Grundströmung zwei zusätzliche hydrodynamische Moden.

Für Vergleiche mit dem Experiment sind nur die radialsymmetrischen Moden $m=0$ interessant, weil auch dort nur mit radialsymmetrischen Schallfeldern gearbeitet wurde.

In der Übersicht 3.7 zeige ich die Lösungen niedrigster Ordnung, also diejenigen mit kleinen Imaginärteilen, im Vergleich zu den Moden vor einer ebenen Wand. Die Zusammenstellung der Moden-Paare ist insofern willkürlich, weil ich nicht getestet habe, ob die Moden bei Deformation der unterschiedlichen Geometrien ineinander übergehen, sofern dies überhaupt sinnvoll möglich ist.

Wie man sieht, sind die Abweichungen der Lösungen im Rohr von den Moden $k_{x}^{ \pm( \pm)}$ganz erheblich, so daß die ebene Wand offenbar eine schlechte Näherung ist.

Da kleine Realteile der Ausbreitungswellenzahl mit kleinen Imaginärteilen der wandnormalen Wellenzahl korrespondieren, ist die instabile Mode nicht besonders stark in der Nähe der Auskleidung lokalisiert. In Abbildung 3.8 ist das Wechseldruckprofil für drei Frequenzen dargestellt. Wie im Falle der ebenen Wand meidet die instabile Mode die Wand für hohe Frequenzen. Die Anfachung in Ausbreitungsrichtung bleibt allerdings hoch.

Ein Vergleich mit den Lösungen $k_{x}^{ \pm( \pm)}$und eine Untersuchung der Ausbreitungsrichtung mit den Methoden aus Anhang A zeigt, daß die hier als Instabilitätsmode bezeichnete Lösung eine stromab laufende, angefachte Welle ist, während die sogenannte stabile Mode offenbar das Pendant der stabilen hydrodynamischen Mode $-(+)$ ist. Ich verzichte bewußt darauf, sie ebenso als hydrodynamisch zu bezeichnen, weil sich diese Lösung anders als die Mode $-(+)$ verhält, siehe dazu auch 3.2.4. Die stromab und die stromauf laufende Mode ähneln den Oberflächenwellen der ebenen Wand, während die beiden Lösungen, die ich hier höhere Moden genannt habe, Vertreter der unendlichen Zahl von näherungsweise rein imaginären Lösungen sind, deren Wellenzahlen sich mit der Frequenz nur wenig verändern.

Mit Hilfe von (3.29) wird aus der Dispersionsbeziehung (3.27) die Bestimmungs- 

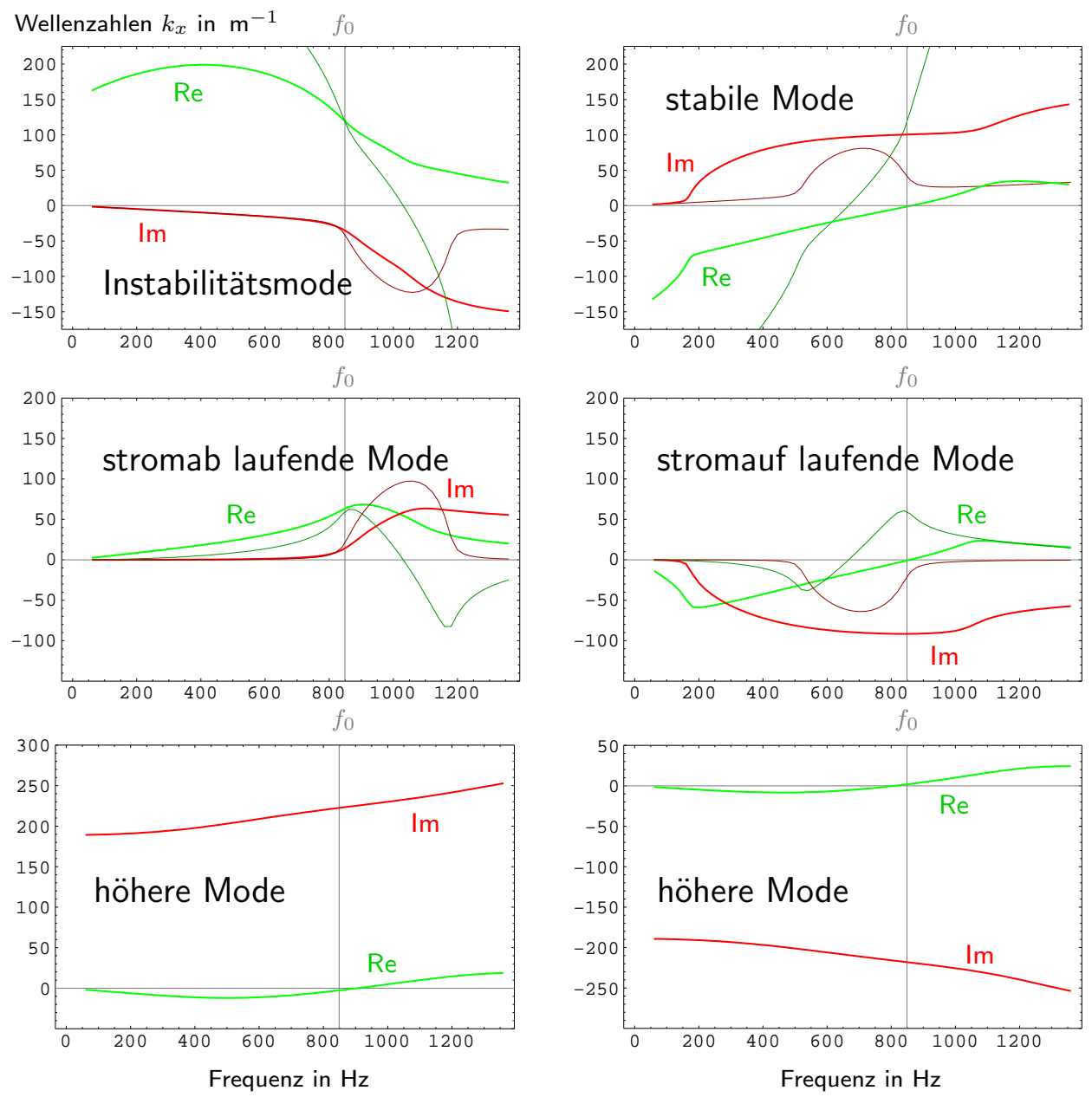

Abbildung 3.7: Vergleich der inkompressiblen Lösungen im Rohr (dicke Linien) mit den Moden $k_{x}^{ \pm( \pm)}$(dünne Linien) bei $u=60 \mathrm{~m} \cdot \mathrm{s}^{-1}$ 


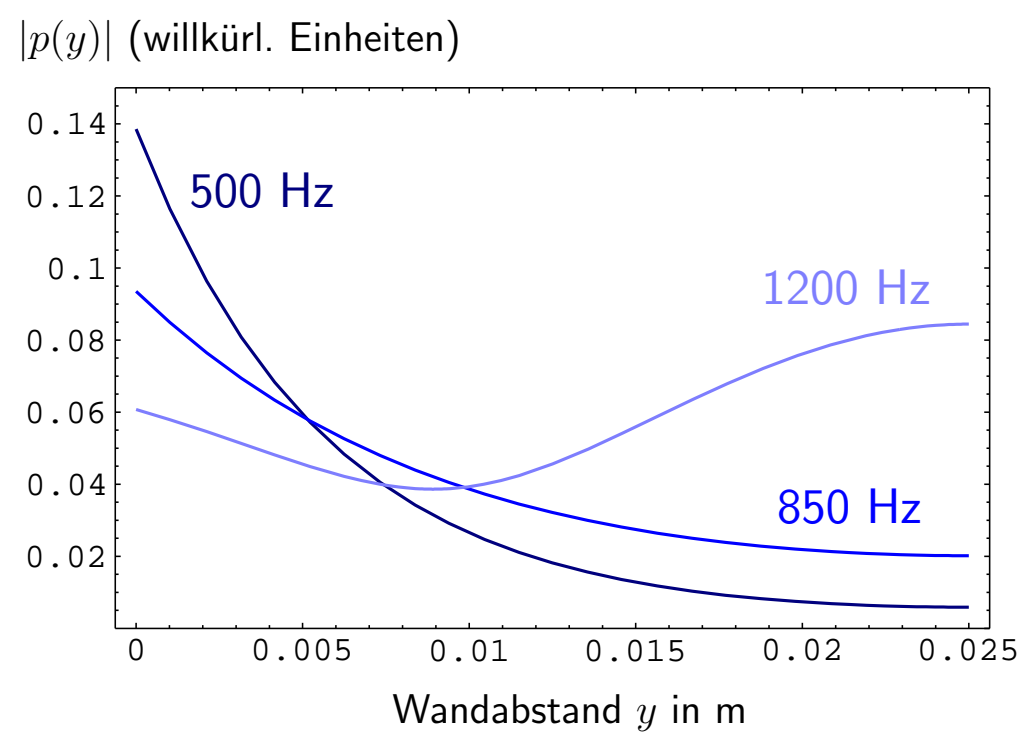

Abbildung 3.8: Wechseldruckamplitude der instabilen Mode in Abhängigkeit vom Wandabstand für drei Frequenzen, $u=60 \mathrm{~m} \cdot \mathrm{s}^{-1}$

gleichung für die kompressiblen Lösungen, die sich ebenfalls nur unwesentlich von den hier präsentierten unterscheiden, weswegen ich auf eine Gegenüberstellung verzichte.

Von Interesse ist noch, wie die instabile Mode für unterschiedliche Strömungsgeschwindigkeiten aussieht. In Abbildung 3.5 hatte ich bereits den Verlauf des Imaginärteils für das Ebene-Wand-Modell dargestellt. In Abbildung 3.9 sind der Real- und Imaginärteil für das Rohr-Modell als Funktionen der Frequenz und der MACH-Zahl zu sehen. Auch hier nimmt der Imaginärteil mit wachsender Machzahl zu, die räumliche Anfachung verringert sich daher, scheinbar im Widerspruch zu den Beobachtungen im Experiment.

Bemerkenswert sind die Verzweigungspunkte bei etwa $1000 \mathrm{~Hz}$, nahe der MACHZahlen 0,1 sowie 0,2. Ihr Auftreten ist nichts Außergewöhnliches, da die implizit über die Dispersionsbeziehung gegebenen Funktionen $k_{x}(f, M a)$ nicht überall stetig sein müssen. Die experimentellen Daten deuten allerdings nicht darauf hin, daß sich die Schallverstärkung bei kritischen MACH-Zahlen sprunghaft ändert. In Abbildung 3.10 sind daher die Verhältnisse am Verzweigungspunkt nahe $M a=0,2$ noch einmal detaillierter dargestellt: Ich zeige den Verlauf der Wellenzahl der instabilen und einer weiteren Mode in der komplexen Ebene. Wie man sieht, 

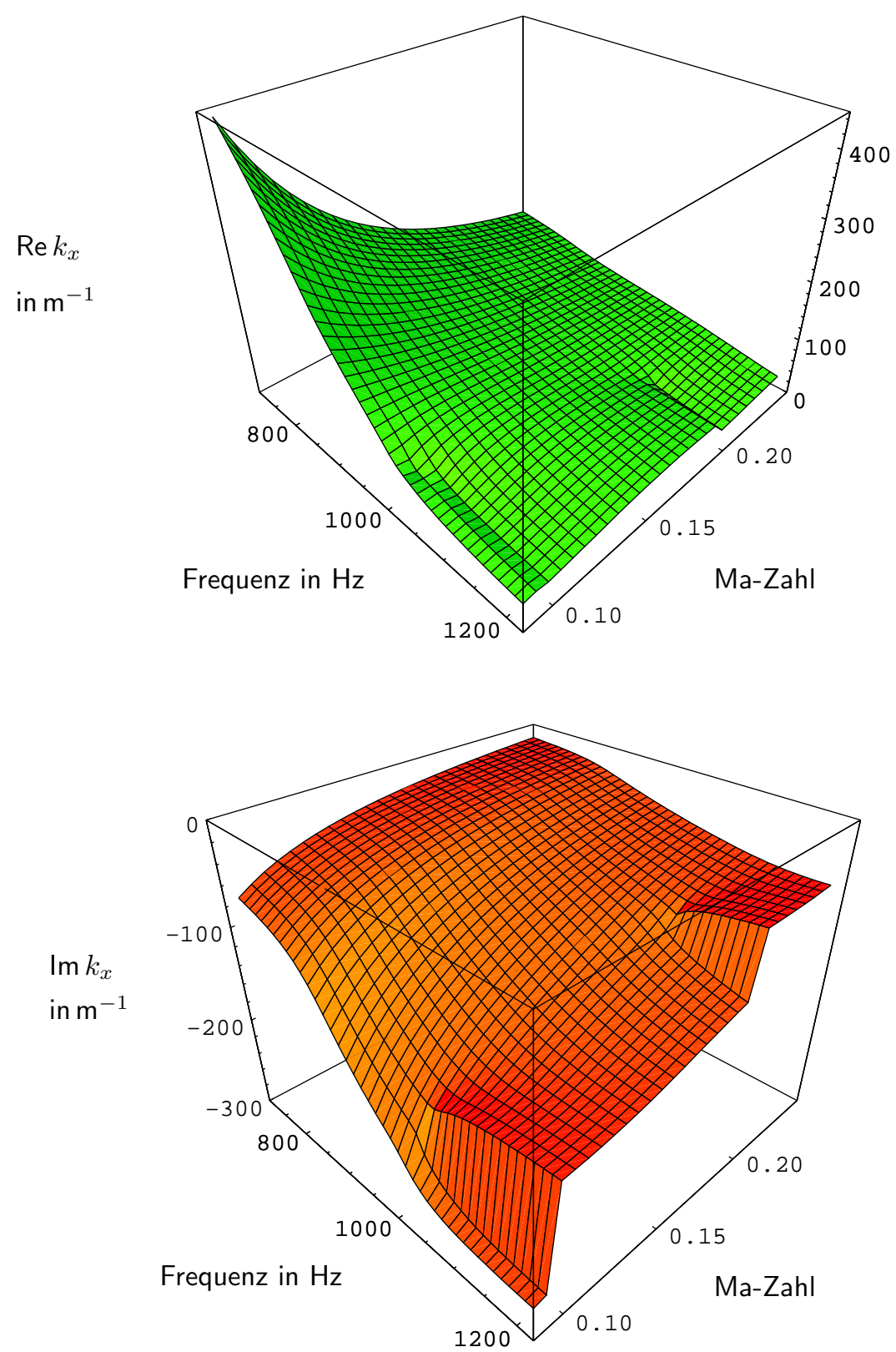

Abbildung 3.9: Die Instabilitätsmode im Rohr in Abhängigkeit von Frequenz und MACH-Zahl, kompressible Rechnung 

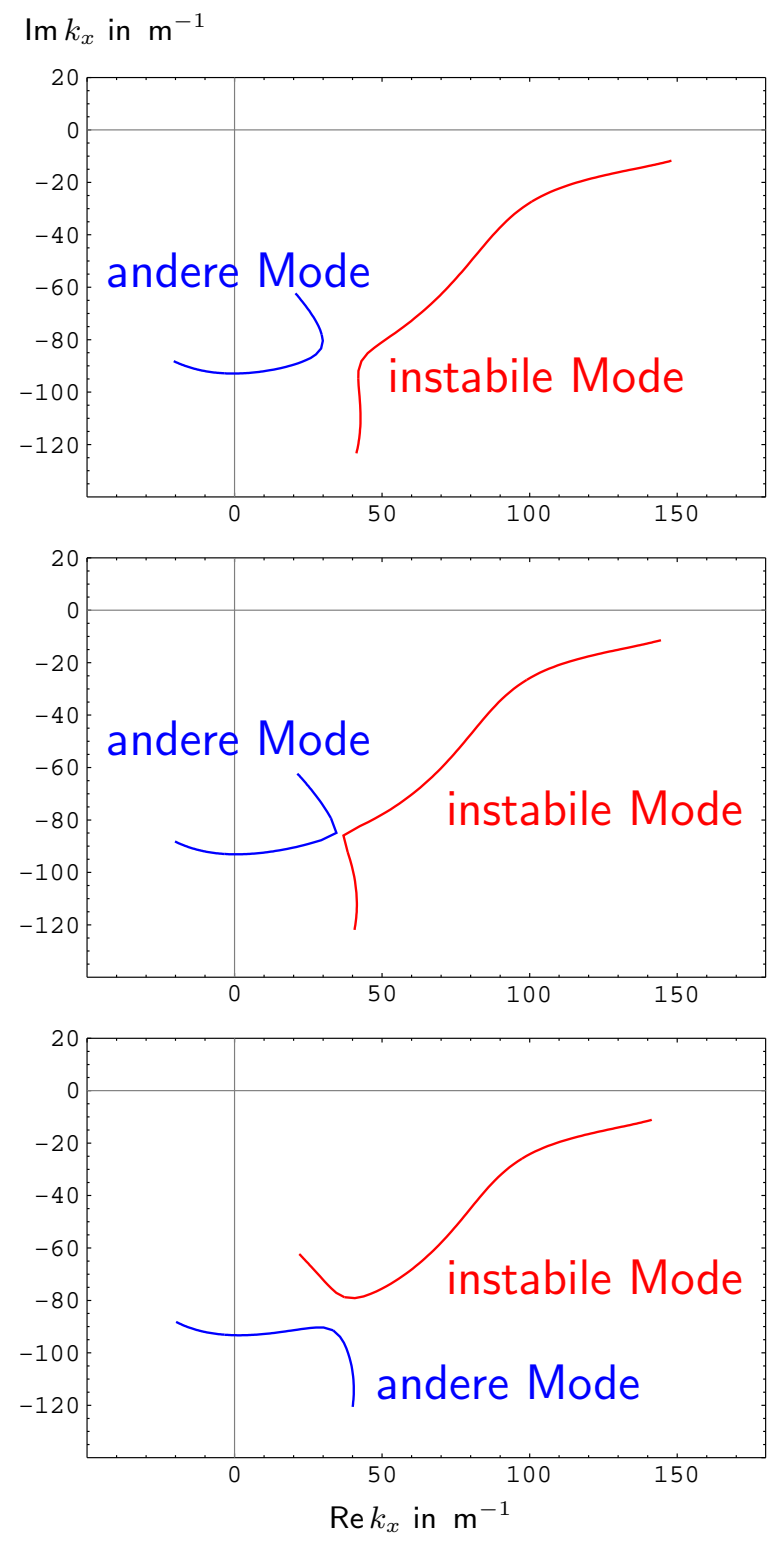

Abbildung 3.10: Wellenzahlen der instabilen und eine weitere Mode unterhalb (oberes Bild), nahe und oberhalb (unteres Bild) der kritischen Strömungsgeschwindigkeit von etwa $70 \mathrm{~m} \cdot \mathrm{s}^{-1}$ in Abhängigkeit von der Frequenz 
„tauschen“ die Moden ab einer gewissen Strömungsgeschwindigkeit ihre Äste für höhere Frequenzen. Das führt einerseits dazu, daß in physikalisch realistischen Lösungen keine Unstetigkeiten auftreten müssen, weil solche Lösungen aus Linearkombinationen aller Moden bestehen, andererseits ist es nicht mehr möglich, Moden zum Beispiel durch ihr Hochfrequenzverhalten zu charakterisieren.

\subsubsection{Flachkanal}

Der dritte Teil dieses Abschnitts ist der Frage gewidmet, inwiefern ein Flachkanal geeigneter Breite als Näherung für einen Kanal mit kreisförmigem Querschnitt dienen kann. Zwar stellt ein Flachkanal keine wesentliche Vereinfachung gegenüber dem Rohr dar, aber es existieren für diese Geometrie schon Streuformeln [10], die ich in Kapitel 5 testen möchte.

Ich verwende in diesem Abschnitt wieder die Koordinaten aus 3.1, wobei jetzt bei $y=D$ eine weitere nachgiebige Wand eingebaut ist. Die Gleichungen (3.4) bis (3.6) gelten unverändert. Um aber die Randbedingung an beiden Wänden simultan zu erfüllen, muß ich nun eine Linearkombination

$$
p, \mathbf{v} \propto\left(A e^{-i k_{y} y}+B e^{+i k_{y} y}\right) e^{-i \omega t+i k_{x} x}
$$

ansetzen, um

$$
\begin{aligned}
& p=-\frac{\omega}{\omega^{\prime}} Z(\omega) v_{y}, y=0 \\
& p=+\frac{\omega}{\omega^{\prime}} Z(\omega) v_{y}, y=D
\end{aligned}
$$

sicherzustellen ${ }^{6}$. Die beiden Randbedingungen (3.31) und (3.32) legen nun zweierlei fest, nämlich das Verhältnis der Linearkombinationsfaktoren A und B,

$$
\begin{aligned}
& A=\left(\omega Z(\omega) k_{y}+\rho\left(\omega-u k_{x}\right)^{2}\right) A_{0} \\
& B=\left(\omega Z(\omega) k_{y}-\rho\left(\omega-u k_{x}\right)^{2}\right) A_{0}
\end{aligned}
$$

mit einer willkürlichen Konstante $A_{0}$ richtiger Dimension, sowie die Dispersionsbeziehung

$$
\left(\omega Z(\omega) k_{y}+\rho\left(\omega-u k_{x}\right)^{2}\right) \mp\left(\omega Z(\omega) k_{y}-\rho\left(\omega-u k_{x}\right)^{2}\right) e^{i k_{y} D}=0
$$

\footnotetext{
${ }^{6}$ Der Unterschied im Vorzeichen ergibt sich wieder aus der Tatsache, daß ein positives $v_{y}$ bei $y=0$ aus der Wand heraus und bei $y=D$ in die Wand hinein zeigt.
} 
Wie in den vorangegangenen Modellen weichen kompressible und inkompressible Rechnung nur durch die Abhängigkeit $k_{y}=k_{y}\left(k_{x}\right)$ voneinander ab. Dabei kann ich das Vorzeichen in dieser Abhängigkeit willkürlich wählen, weil es Gleichung (3.34) aus Symmetriegründen nicht verändert.

Das Vorzeichen in (3.34) dagegen unterscheidet zwischen zwei Familien physikalisch unterschiedlicher Moden. Ausgedrückt durch die Amplituden $A$ und $B$ lautet die Dispersionsbeziehung

$$
A \mp B e^{i k_{y} D}=0
$$

was für das Druckfeld der Mode

$$
p \propto\left(e^{i k_{y}(D-y)} \pm e^{+i k_{y} y}\right) e^{-i \omega t+i k_{x} x}
$$

ergibt. Das Vorzeichen gibt daher an, ob eine Lösung symmetrisch oder antisymmetrisch ist, ob also $p(0)=p(D)$ oder $p(0)=-p(D)$ ist.

In Abb. 3.11 sind die symmetrischen Lösungen niedrigster Ordnung den entsprechenden Moden im Rohr gegenübergestellt. Wie man sieht, ist die Übereinstimmung nicht besonders gut, lediglich der prinzipielle Verlauf der Lösungen stimmt überein. Diese Übereinstimmung läßt sich durch Veränderung der Kanalbreite nicht entscheidend verbessern. Trotzdem werde ich diese Moden zunächst für die Streurechnung in Kapitel 5 benutzen.

Vergrößert man die Strömungsgeschwindigkeit, so steigt auch im Flachkanal der Imaginärteil auf kleinere negative Werte — wie im Rohr. Diese Abnahme der räumlichen Anfachung mit wachsender Strömungsgeschwindigkeit scheint also nicht von den geometrischen Details abzuhängen, sondern ist offenbar eine Eigenschaft des Instabilitätsmechanismus selbst.

In Abb. 3.12 zeige ich die symmetrischen Moden im Vergleich zu den entsprechenden antisymmetrischen Lösungen. Wie man sieht, ist die antisymmetrische Instabilitätsmode weniger angefacht. Für die Deutung des Experimentes sind diese Lösungen zwar ohne Belang, da dort keine antisymmetrischen Schallfelder erzeugt wurden, allerdings würden sie bei Versuchen eine Rolle spielen, durch antisymmetrische Anregung der Instabilität zu verhindern, daß sich der Steuerschall in den schallharten Abschnitten ausbreitet, wo derartige Moden nicht ausbreitungsfähig sind. 

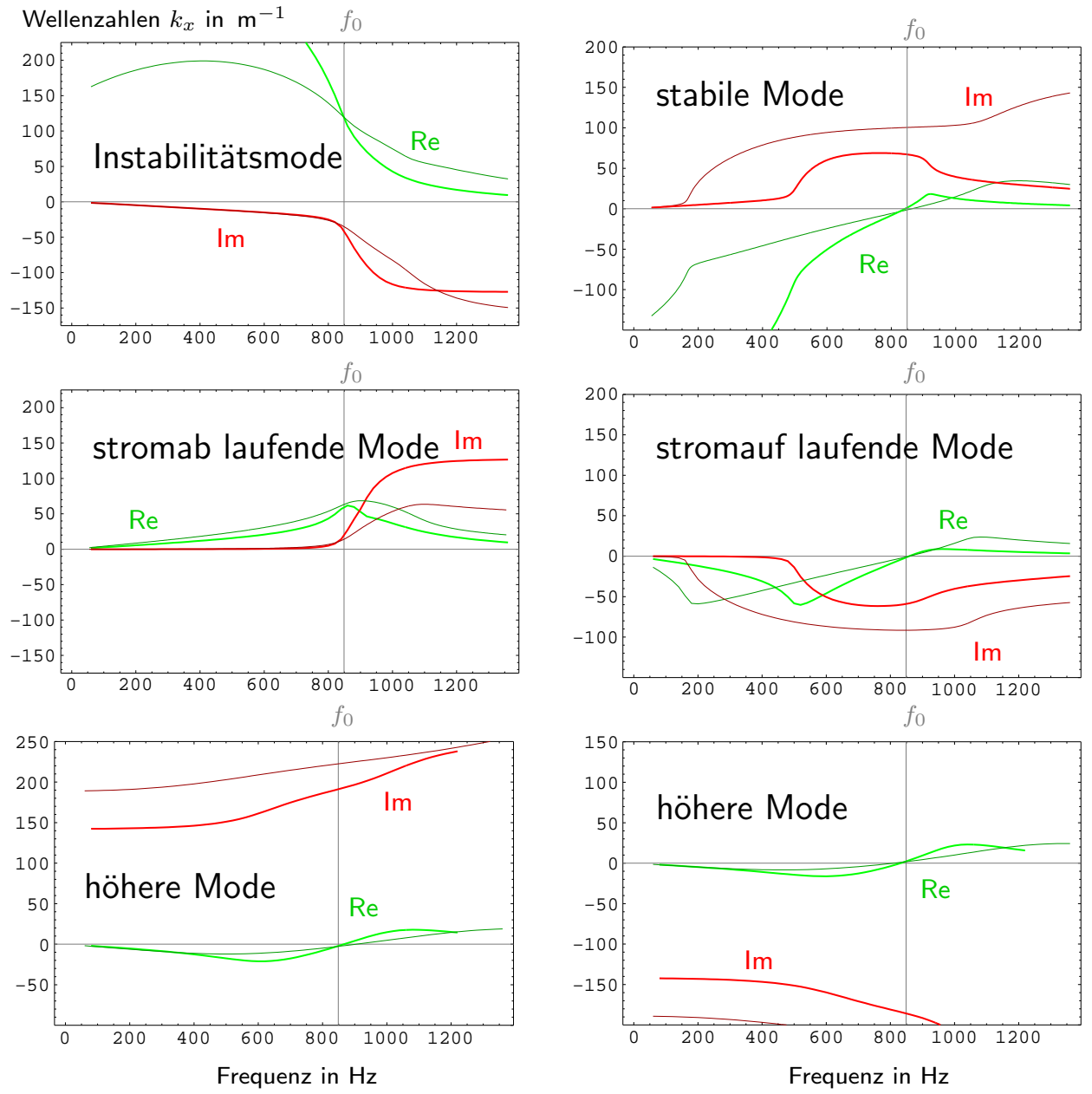

Abbildung 3.11: Vergleich der inkompressiblen, symmetrischen Lösungen im Flachkanal (dicke Linien) mit den Lösungen im Rohr (dünne Linien) bei $u=60 \mathrm{~m} \cdot \mathrm{s}^{-1}$. Breite des Flachkanals: $D=50 \mathrm{~mm}$. 

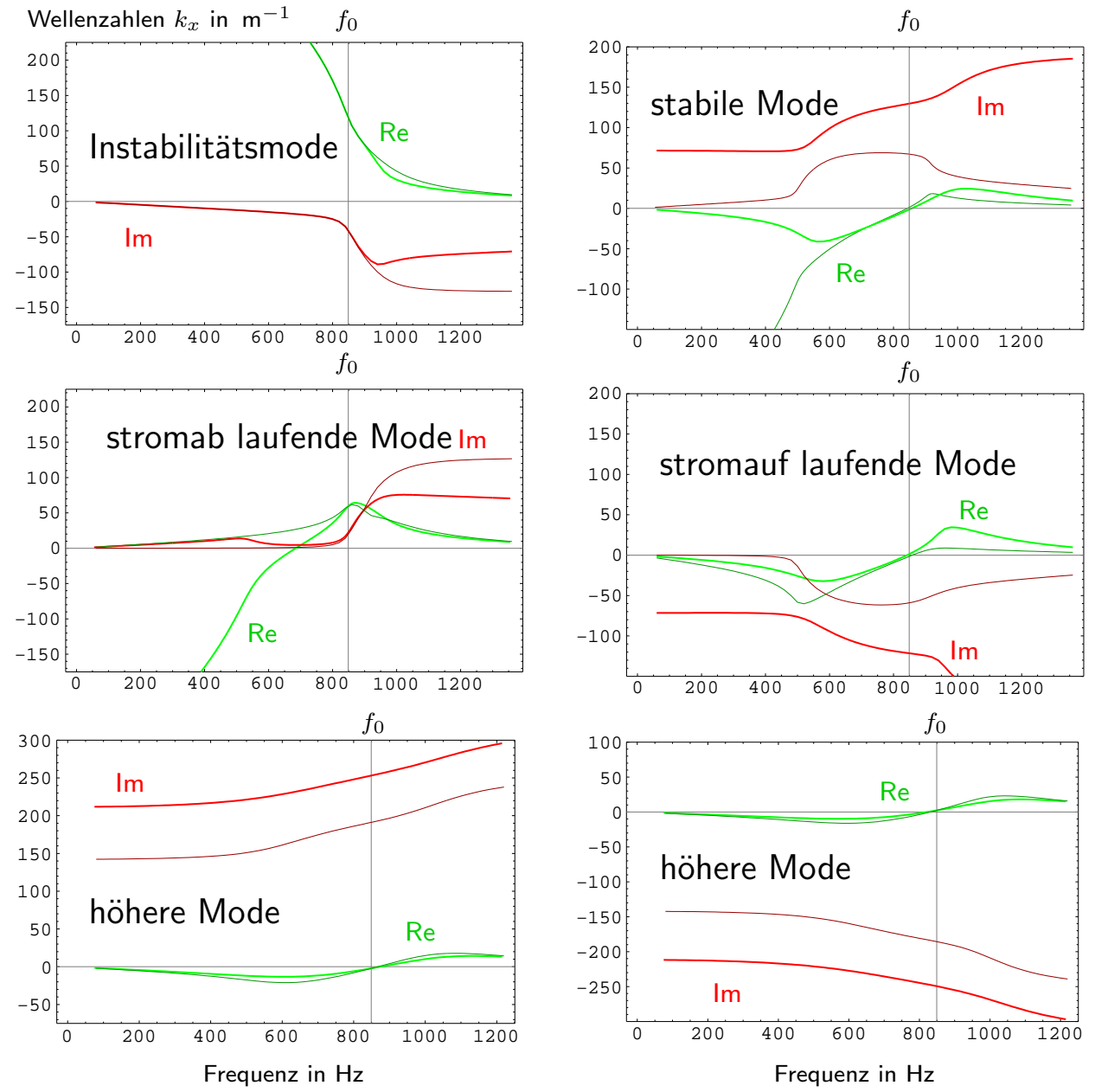

Abbildung 3.12: Vergleich der antisymmetrischen Lösungen im Flachkanal (dicke Linien) mit den symmetrischen (dünne Linien) bei $u=60 \mathrm{~m} \cdot \mathrm{s}^{-1}$. Breite des Flachkanals: $D=50 \mathrm{~mm}$. 


\begin{tabular}{|l|l|l|}
\hline Kriterium & $k_{x}^{ \pm(+)}$ & $k_{x}^{ \pm(-)}$ \\
\hline $\begin{array}{l}u \rightarrow 0 \text { (keine Grundströmung) } \\
Z \rightarrow \infty \text { (schallharte Wand) }\end{array}$ & divergieren & $k_{x}^{ \pm(-)} \rightarrow \pm \frac{i \omega \rho}{Z}$ \\
$\omega \rightarrow 0$ (stationär) & divergieren & $k_{x}^{ \pm(-)} \rightarrow 0$ \\
"Phasengeschwindigkeit“" & $k_{x}^{ \pm(+)} \rightarrow \pm \frac{c^{2}}{u^{2} T}$ & $k_{x}^{ \pm(-)} \rightarrow 0$ \\
\hline
\end{tabular}

Tabelle 3.2: Eigenschaften der Moden $k_{x}^{ \pm( \pm)}$

\subsubsection{Zum Begriff der hydrodynamischen Mode}

Der Unterschied zwischen sogenannten „hydrodynamischen“ Moden einerseits und „akustischen" beziehungsweise Oberflächenmoden andererseits ist im Modell des Abschnittes 3.1 klar erkennbar.

Tabelle 3.2 zeigt, daß zwei Lösungen-darunter die Instabilitätsmode - ohne Strömung (und nachgiebige Wände) nicht existieren, im Grenzfall verschwindender Frequenz in stationäre Strömungen übergehen und ihre Phasenfronten mit einer Geschwindigkeit laufen, die in der Größenordnung von $u$ liegt. Sie entsprechen also recht genau der Vorstellung, die man sich von einer hydrodynamischen Welle macht.

Im Falle der Kanäle aus den Abschnitten 3.2.2 und 3.2.3 ist eine strikte Unterscheidung nicht mehr möglich.

Das wichtigste Kriterium ist sicherlich, ob die Mode auch für verschwindende Grundströmung noch existiert. Dazu habe ich in Abbildung 3.13 den Verlauf der Wellenzahlen zweier Lösungen bei je zwei festen Frequenzen für $u \rightarrow 0$, beginnend mit $u=60 \mathrm{~m} \cdot \mathrm{s}^{-1}$, in die komplexe Ebene eingezeichnet. Der Deutlichkeit halber sind zwei Ausschnitte noch einmal vergrößert dargestellt.

Wie man sieht, hängt die Konvergenz für $u \rightarrow 0$ von der Frequenz ab: Für niedrige divergieren die Wellenzahlen der Instabilitäts- und der ersten höheren Mode, für hohe Frequenzen bleiben sie endlich. Die kritische Frequenz scheint nahe der Tiefenresonanz der Wand zu liegen, falls es nicht die Resonanzfrequenz 

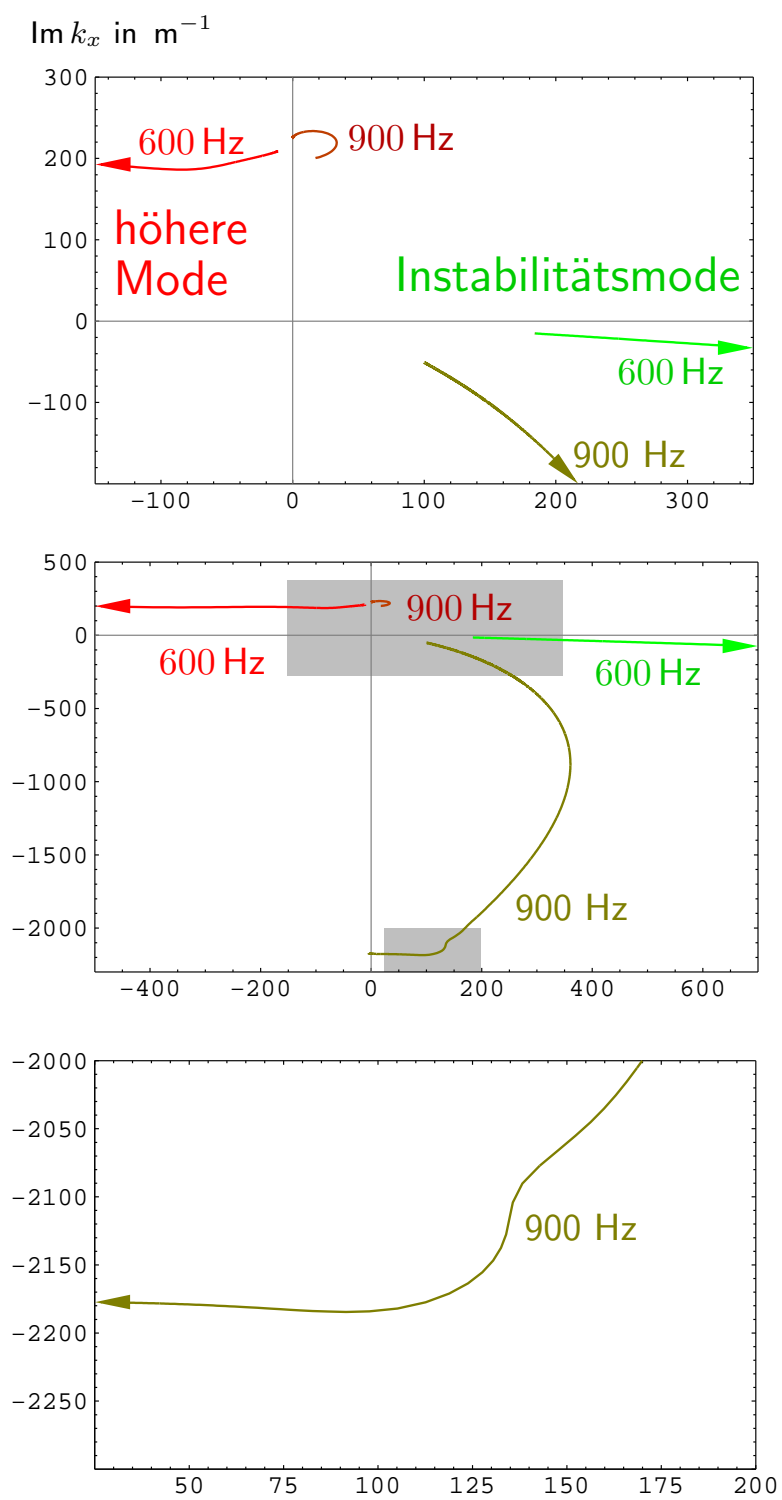

$\operatorname{Re} k_{x}$ in $\mathrm{m}^{-1}$

Abbildung 3.13: Ausbreitungswellenzahlen der Instabilitätsmode (grün) und der ersten höheren, stromab laufenden Mode (rot) im Rohr für sinkende Strömungsgeschwindigkeit, beginnend bei $60 \mathrm{~m} \cdot \mathrm{s}^{-1}$ 
selbst ist.

Ebenso überraschend ist die Beobachtung, daß nicht etwa die der stabilen hydrodynamischen Mode $-(+)$ ähnlichste Lösung divergentes Verhalten für $u \rightarrow 0$ zeigt, sondern eine höhere Mode.

Daß die Verhältnisse in ausgekleideten Kanälen —ob Rohr oder Flachkanalanders sind als vor einer einzelnen Wand, zeigt sich auch in den Beträgen der bereits eingeführten Geschwindigkeit $v_{\mathrm{ph}}$, wie Abbildung 3.14 zeigt. Der Unterschied zwischen den ursprünglich als „hydrodynamisch“ und als „akustisch“ angesehenen Moden ist längst nicht so groß wie bei einer ebenen Wand (Abb. 3.3). Dieser geringe Unterschied in der Geschwindigkeitsskala und die Beobachtungen aus dem Grenzfall $u \rightarrow 0$ lassen sich meiner Ansicht nach so interpretieren, daß eine strenge Trennung zwischen hydrodynamischen und akustischen beziehungsweise Oberflächenwellen in Kanälen mit einer engen Wechselwirkung zwischen Medium, Strömung und nachgiebiger Wand nicht möglich ist.

\subsubsection{Zusammenfassung}

Die in diesem Abschnitt diskutierten Abwandlungen des Modells aus Abschnitt 3.1 ergeben folgendes Bild:

- Die Kompressibilität der Luft hat auf die Dispersionsbeziehungen nur geringen Einfluß, wenn man sich auf die experimentell erreichten Strömungsgeschwindigkeiten beschränkt. Ein einfaches Modell sollte daher ohne Kompressibilität auskommen und die Strömungsgeschwindigkeit $u$ sollte die maßgebliche Geschwindigkeit sein. $c$ ginge in die Gleichungen nur indirekt über die Wandimpedanz ein.

- Das Verhalten der Moden im Rohr weicht erheblich von den Lösungen an einer ebenen Wand ab. Das gilt auch für die instabile Lösung, die zur Kanalmitte hin nur wenig abfällt.

- In keinem der gerechneten Modelle ist ein Anhaltspunkt dafür zu finden, warum die Verstärkung bei hohen Frequenzen zusammenbricht. Die Instabilitätsmode scheint auch noch für weit höhere Frequenzen angefacht zu sein. Weiterhin fällt der Realteil der Wellenzahl dort mit steigender Frequenz ab, ein Verhalten, das man aufgrund der Drucksondenmessungen von Brandes (siehe [3], Abb. 4.16b) so nicht erwarten würde.

- In allen Modellen nimmt die räumliche Anfachung ab, wenn die Strömungsgeschwindigkeit erhöht wird. 

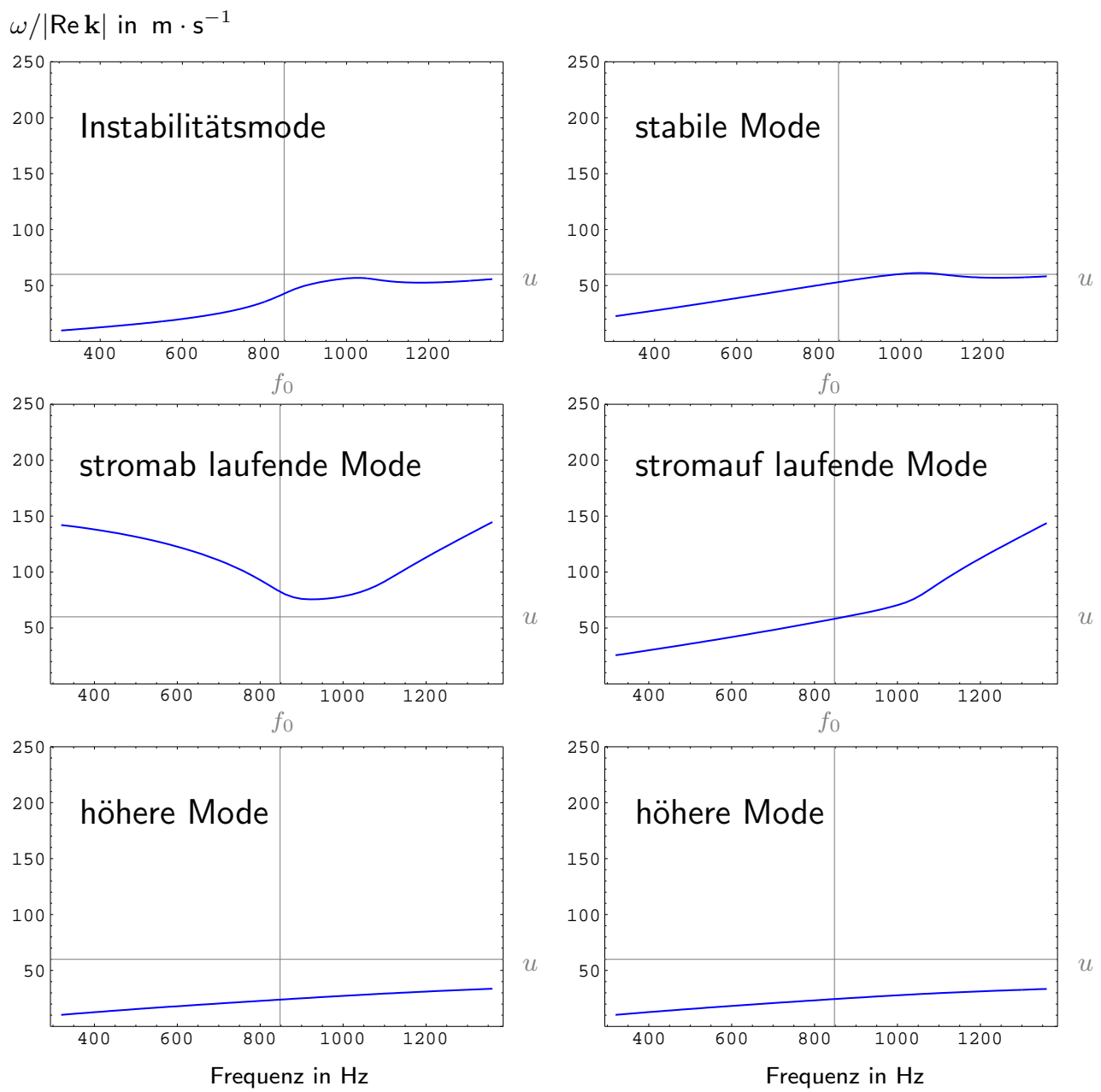

Abbildung 3.14: Betrag von $v_{\mathrm{ph}}$ für die sechs Lösungen niedrigster Ordnung im Rohr bei einer Gleichströmung von $60 \mathrm{~m} \cdot \mathrm{s}^{-1}$ 


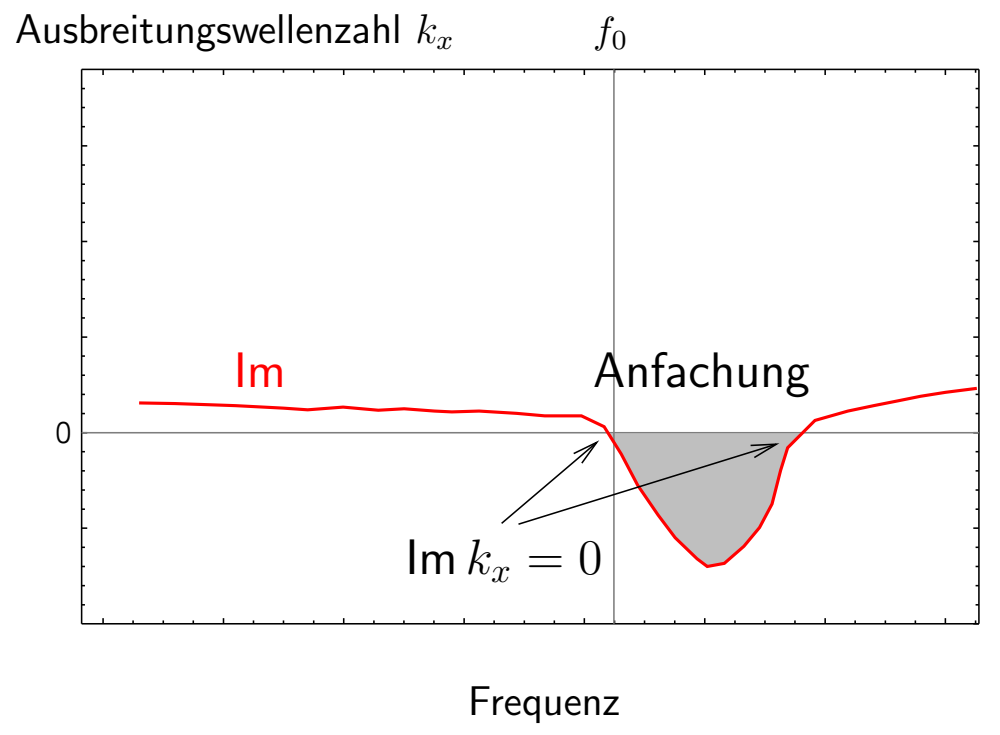

Abbildung 3.15: Hypothetischer Verlauf des Imaginärteils der instabilen Mode

- Die Moden in einem Flachkanal vergleichbarer Breite verlaufen etwas anders als in einem Rohr, jedoch stimmt das prinzipielle Verhalten der Wellenzahlen überein. Daher erscheint es gerechtfertigt, die Methode aus [10], die ich für die Ermittlung der Streufaktoren benutzen möchte, wie die Autoren an dieser Geometrie zu testen.

Mit den bisher vorgenommenen Idealisierungen ist die Suche nach einer Instabilitätsmode, die den Verlauf des Transmissionsfaktors reproduziert (siehe Abb. 3.15), allerdings von vornherein zum Scheitern verurteilt: Es ist nämlich nicht möglich, daß eine Lösung (in Abhängigkeit von der reellen Frequenz) stetig von räumlicher Dämpfung zu räumlicher Anfachung und zurück wechselt, also einen nur endlich breiten Frequenzbereich mit negativem Imaginärteil von $k_{x}$ aufweist.

Der Grund dafür liegt darin, daß für reelles $k_{x}$, wie es am Beginn und am Ende des Anfachungsbereiches vorliegen müßte, die Querwellenzahl $k_{y}$ (beziehungsweise $k_{r}$ ) entweder rein imaginär oder rein reell wäre - letzteres auch nur bei kompressibler Rechnung, wenn folgende Ungleichung erfüllt wird: 


$$
\begin{aligned}
k_{y}^{2} & >0 \\
-\frac{\omega / c}{1-M a}< & k_{x}<\frac{\omega / c}{1+M a}
\end{aligned} \Leftrightarrow
$$

Für imaginäre Querwellenzahl sieht man anhand der Dispersionsbeziehungen (3.10), (3.19), (3.27) und (3.34) sofort, daß $Z$ dann ebenfalls rein imaginär sein müßte, was wegen des immer vorhandenen resistiven Terms für keine Frequenz der Fall ist.

Falls die Querwellenzahl reell sein sollte, folgt für Rechteckkanal und Rohr aus $\operatorname{Im} k_{x}=0$ wiederum $\operatorname{Re} Z=0$. An der ebenen Wand folgt dagegen, daß dann die Wandimpedanz reell ist - was zwar in der Resonanzfrequenz, aber niemals für eine höhere Frequenz (unterhalb der nächsten Resonanz) der Fall sein kann.

Mit weiteren resistiven Mechanismen, die sich mathematisch durch zusätzliche komplexe Terme in den Dispersionsbeziehungen bemerkbar machen würden, wäre diese Argumentation hinfällig und das in Abb. 3.15 beschriebene Verhalten der Wellenzahl denkbar.

\subsection{Zähigkeitseffekte}

In den bisher vorgestellten Modellen ist der Einfluß des turbulenten Geschwindigkeitsprofils auf die Wellenlösungen unberücksichtigt geblieben. Dieses zu vernachlässigen ist zwar mit Sicherheit eine schlechte Näherung, erleichtert aber die Analyse der Zahl und Art der existierenden Moden.

Wegen der verhältnismäßig hohen REYNOLDS-Zahlen im Verstärkungsbereich ist von vornherein klar, daß der turbulente Impulstransport den viskosen bei weitem überwiegt. Andererseits ist das Strömungsprofil schon recht steil und die Moden gemäß den vorhergegangenen Untersuchungen nicht besonders stark an der Wand lokalisiert. Es liegt daher nahe, als nächsten Schritt eine Modellierung mit einer mittleren turbulenten Zähigkeit, aber ohne turbulentes Strömungsprofil zu versuchen, um zumindest abschätzen zu können, mit welcher Tendenz sich die Dispersionsbeziehungen verändern.

\subsubsection{Die Größe der turbulenten Zähigkeit}

Zur turbulenten Rohrströmung liegen viele Messungen und theoretische Modelle vor. Die Mischungsvorgänge in einer solchen Strömung führen zu einem Verhalten, 
das dem eines Mediums mit stark erhöhter Zähigkeit $\nu_{\mathrm{t}}$, auch Wirbelviskosität genannt, ähnelt, die erst unmittelbar an der Wand auf den Wert der viskosen Zähigkeit $\nu$ absinkt.

Die abgeleiteten Gesetzmäßigkeiten gelten zunächst nur bei stationären turbulenten Strömungen, für die sich das gemittelte Geschwindigkeitsfeld zeitlich nicht verändert. Ich untersuche aber Wechselströmungen; meine erste Annahme ist daher, daß für diese die turbulente Zähigkeit einen zumindest annähernd gleichen Wert hat (Approximation als quasistationäre Strömung).

Physikalisch bedeutet diese Annahme, daß die Wechselströmungen deutlich langsamer verlaufen als die für den Impulstransport verantwortlichen Mischbewegungen.

Die zweite Annahme betrifft die Ortsabhängigkeit von $\nu_{\mathrm{t}}$. Für ein glattes Rohr hängt diese in der folgenden Weise vom Wandabstand ab,

$$
\nu_{\mathrm{t}}=\frac{\kappa R u_{\tau}}{6}\left(1-\frac{r^{2}}{R^{2}}\right)\left(1+\frac{r^{2}}{R^{2}}\right)
$$

siehe Gleichung 17.133 in [21]. Dabei ist $\kappa$ die KÁrmán-Konstante und $u_{\tau}$ die Wandschubspannungsgeschwindigkeit. Das führt zu einem Verlauf wie in Abbildung 3.16.

Um ein ortsabhängiges $\nu_{\mathrm{t}}$ und die dann nur noch numerisch durchführbare Integration der Differentialgleichungen zu vermeiden, möchte ich für Wechselströmungen, die den gesamten Querschnitt ausfüllen, eine ortsunabhängige mittlere Zähigkeit annehmen.

Die dritte Näherung betrifft das sich im nachgiebigen Rohr einstellende Strömungsprofil. Diese hängt, wie in [21] dargestellt, von der Beschaffenheit der Wand ab. Selbst wenn das Profil für die im Meßobjekt vorliegende Auskleidung bekannt wäre, hätte man davon keinen großen Nutzen, weil die Länge des Meßobjektes weniger als zwei Rohrdurchmesser beträgt und sich die Strömung, aus dem glatten Rohr kommend, auf dieser Wegstrecke noch nicht neu eingestellen kann. Ich kann daher genauso gut davon ausgehen, daß innerhalb der verhältnismäßig kurzen ausgekleideten Strecke ein ähnliches Strömungsprofil wie im glatten Rohr vorliegt, und mit dieser Annahme die Formel 17.127 aus [21] für den Zusammenhang zwischen Schubspannungs- und der über den Querschnitt gemittelten Geschwindigkeit benutzen:

$$
\bar{u}=\frac{u_{\tau}}{\kappa} \ln \left(\frac{R u_{\tau}}{\nu}\right)+1,96 u_{\tau}
$$




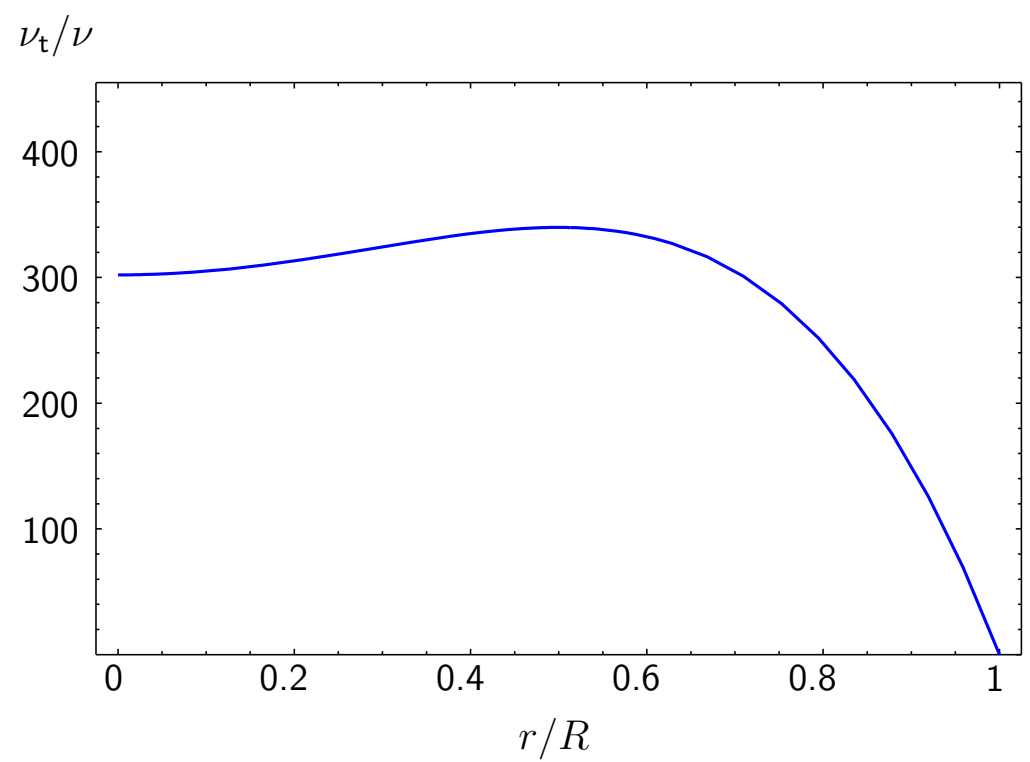

Abbildung 3.16: Radiale Abhängigkeit der turbulenten Viskosität für $u_{\tau}=2,67 \mathrm{~m} \cdot \mathrm{s}^{-1}$ (entspricht $\bar{u}=60 \mathrm{~m} \cdot \mathrm{s}^{-1}$ )

In Abb. 3.17 habe ich diesen Zusammenhang (für den im Experiment verwendeten Rohrradius und Luft unter Laborbedingungen) dargestellt.

Es ist allerdings von vornherein klar, daß für einen gegebenen Massenfluß (beziehungsweise eine gegebene, über den Querschnitt gemittelte Strömungsgeschwindigkeit) eine deutlich höhere Wandschubspannung bzw. Schubspannungsgeschwindigkeit $u_{\tau}$ notwendig ist, da der Druckabfall im kassettierten Rohrstück bekanntermaßen größer ist.

Unter der Voraussetzung, daß die drei vorangegangenen Annahmen für die durchströmten Meßobjekten näherungsweise anwendbar sind, ergibt sich für die interessanten Strömungsgeschwindigkeiten eine turbulente Zähigkeit, die einige hundertmal größer als die viskose ist.

\subsubsection{Ebene Wand}

Im Falle endlicher Zähigkeit tritt neben die Kontinuitätsgleichung

$$
\operatorname{div} \mathbf{v}=0
$$




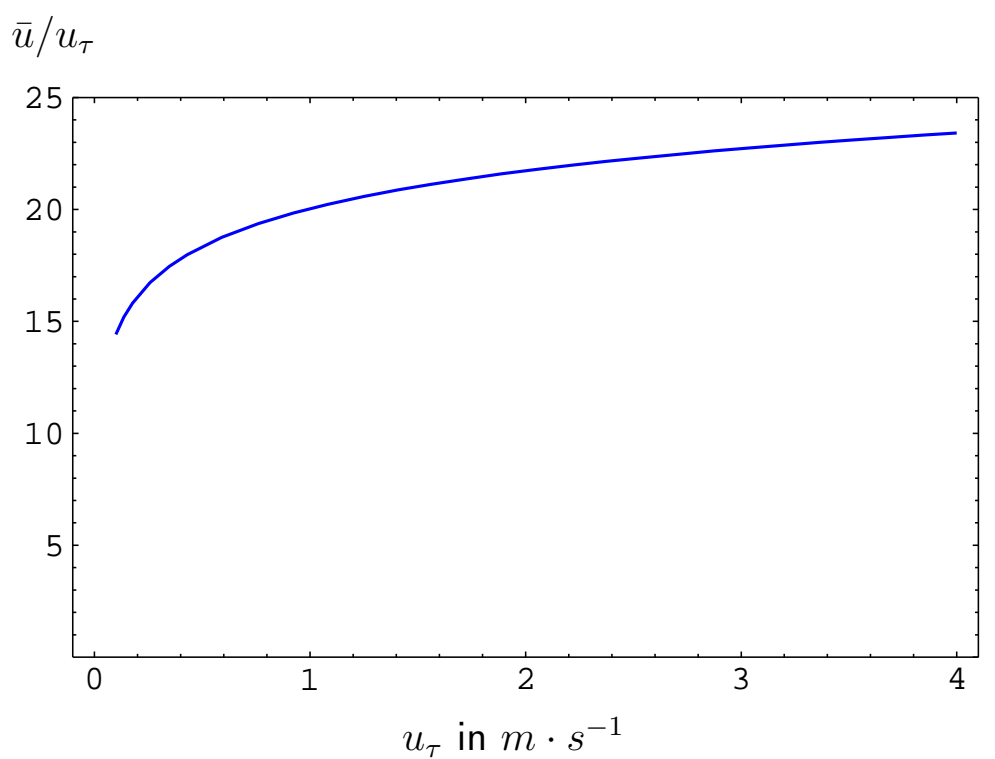

Abbildung 3.17: Über den Querschnitt gemittelte Grundströmungsgeschwindigkeit in Abhängigkeit von der Schubspannungsgeschwindigkeit

nun die NAVIER-STOKES-Gleichung

$$
\left(\partial_{t}+u \partial_{x}\right) \mathbf{v}=-\frac{1}{\rho} \nabla p+\nu_{\mathrm{t}} \Delta \mathbf{v}
$$

in der $\nu_{\mathrm{t}}$ eine turbulente Viskosität sein soll, die viel größer als die kinematische Viskosität der Luft ist.

In der inkompressiblen Näherung verschwindet die Divergenz des Geschwindigkeitsfeldes und Gleichung (3.39) liefert

$$
\Delta p=0
$$

Da ich hier zeitlich und räumlich harmonische Lösungen untersuche, sind nur die Fälle $p \equiv$ const., also ohne Beschränkung der Allgemeinheit

$$
p \equiv 0
$$

oder

$$
\Delta=k_{x}^{2}+k_{y}^{2}=0
$$


möglich.

Im ersten Fall kann aus der NAVIER-STOKES-Gleichung folgende Beziehung für die Wellenzahlkomponenten $k_{x}, k_{\mathrm{St}} \doteq k_{y}$ hergeleitet werden:

$$
\nu_{\mathrm{t}}\left(k_{x}^{2}+k_{\mathrm{St}}^{2}\right)-i\left(\omega-u k_{x}\right)=0
$$

Diese Wellen, deren Dynamik nicht durch Druckkräfte, sondern nur durch die Zähigkeit bestimmt wird, sind die sogenannten STOKES-Wellen, deren wandnormale Wellenzahlkomponente dann folgende Gleichung erfüllt:

$$
k_{\mathrm{St}}=i \sqrt{k_{x}^{2}-\frac{i\left(\omega-u k_{x}\right)}{\nu_{\mathrm{t}}}}
$$

Mit der herkömmlichen Definition der komplexen Wurzel (bei der der Schnitt auf der negativen reellen Halbachse liegt und die Wurzel so einen nichtnegativen Realteil hat), ist der Imaginärteil von $k_{\mathrm{St}}$ stets - physikalisch sinnvoll-größer (oder im Grenzfall gleich) Null.

Das Schnellefeld dieser Lösungen hat dann die Gestalt

$$
\begin{aligned}
& v_{x}=\quad v_{\mathrm{St}}^{0} \cdot e^{-i \omega t+i k_{x} x+i k_{\mathrm{St}} y} \\
& v_{y}=-\frac{k_{x}}{k_{\mathrm{St}}} v_{\mathrm{St}}^{0} \cdot e^{-i \omega t+i k_{x} x+i k_{\mathrm{St}} y}
\end{aligned}
$$

wobei $v_{\mathrm{St}}^{0}$ eine willkürliche Amplitude ist.

Für die zweite Klasse von Wellen, die die herkömmlichen zähigkeitsfreien Moden umfaßt, folgt aus (3.40) wieder die Beziehung

$$
k_{y}^{ \pm}= \pm i k_{x}
$$

und das Schall- und Schnellefeld haben die Gestalt

$$
\begin{aligned}
p & =p_{0} \cdot e^{-i \omega t+i k_{x} x+i k_{y} y} \\
v_{x} & =\frac{k_{x} p_{0}}{\rho\left(\omega-u k_{x}\right)} \cdot e^{-i \omega t+i k_{x} x+i k_{y} y} \\
v_{y} & =\frac{k_{y} p_{0}}{\rho\left(\omega-u k_{x}\right)} \cdot e^{-i \omega t+i k_{x} x+i k_{y} y}
\end{aligned}
$$


STOKES- und Ausbreitungswellen müssen nun so linearkombiniert werden, daß die Haftbedingung und die Impedanzbeziehung

$$
\begin{array}{llrl}
v_{x} & =0 & , y & =0 \\
v_{y}=-\frac{\omega^{\prime}}{\omega} \frac{p}{Z(\omega)} & , y & =0
\end{array}
$$

gleichzeitig erfüllt werden. Bei fester Frequenz und Machzahl erfordert das die gleiche Ausbreitungswellenzahl $k_{x}$ für die zu kombinierenden Wellen. Die Haftbedingung (3.50) legt das Amplitudenverhältnis der Schnellen in $x$-Richtung fest. Die Impedanzbeziehung (3.51) führt dann zur Dispersionsrelation

$$
1+\left(\frac{\omega-u k_{x}}{\omega}\right)^{2} \frac{\rho \omega}{k_{y}^{ \pm} Z}-\frac{k_{x}^{2}}{k_{y}^{ \pm} k_{\mathrm{St}}}=0
$$

Für verschwindende Zähigkeit geht der dritte Term auf der linken Seite gegen Null und die Dispersionsbeziehung geht in Gleichung (3.10) über.

In Abbildung 3.18 ist zu sehen, wie sich der Verlauf der Instabilitätsmode bei hoher Zähigkeit verändert. Der hier verwendete Wert ergibt sich aus den Gleichungen (3.36) und (3.37) für $u=60 \mathrm{~m} \cdot \mathrm{s}^{-1}$ (dort mit $\bar{u}$ bezeichnet).

Der Einfluß von $\nu_{\mathrm{t}}$ ist verhältnismäßig gering. Die meiner Meinung nach entscheidende Änderung ist das nun gedämpfte Verhalten der Mode für niedrige Frequenzen (grau unterlegt), wobei sich der Nulldurchgang des Imaginärteils für größerer Viskositäten weiter in Richtung der Resonanzfrequenz verschiebt.

Allerdings deutet auch in diesem Modell nichts darauf hin, daß die räumliche Anfachung für hohe Frequenzen (oberhalb des von BRANDES beobachteten Frequenzbandes) zusammenbricht.

\subsubsection{Rohr}

Die Dispersionsbeziehung für die Rohrgeometrie herzuleiten ist ebenfalls geschlossen möglich. Da es die Rechnung nicht wesentlich erschwert, berücksichtige ich nun auch die Kompressibilität der Luft. Wieder untersuche ich nur längs der Rohrachse rotationssymmetrische Moden (keine $\phi$-Abhängigkeit).

Die Grundgleichungen lauten jetzt 


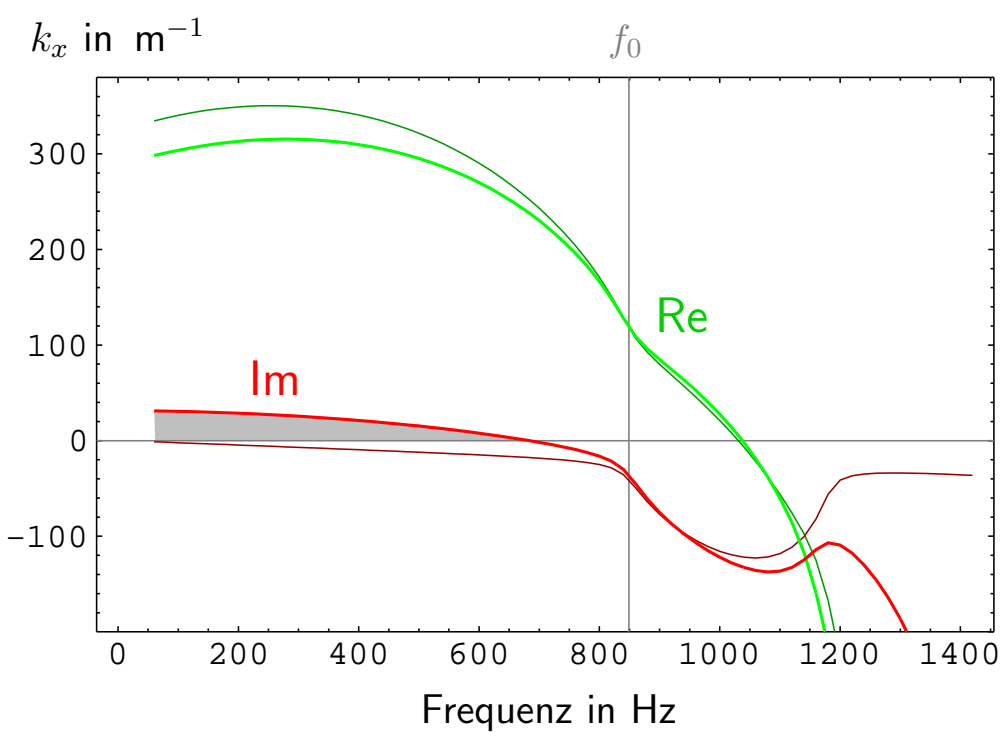

Abbildung 3.18: Instabilitätsmode ohne (dünne Linien) und mit turbulenter Zähigkeit $\nu_{\mathrm{t}}=300 \nu$ (dicke Linien) bei $u=60 \mathrm{~m} \cdot \mathrm{s}^{-1}$

$$
\begin{aligned}
& \frac{1}{c^{2}}\left(\partial_{t}+u \partial_{x}\right) p+\rho \operatorname{div} \mathbf{v}=0 \\
& \left(\partial_{t}+u \partial_{x}\right) \mathbf{v}=-\frac{1}{\rho} \nabla p+\nu_{\mathrm{t}}\left(\Delta \mathbf{v}-\frac{v_{r}}{r^{2}} \mathbf{e}_{r}\right)
\end{aligned}
$$

mit dem LAPLACE-Operator, der Divergenz und dem Gradient in Zylinderkoordinaten. Die Divergenz der NAVIER-STOKES-Gleichung führt unter Verwendung von (3.53) auf die Wellengleichung

$$
\frac{1}{c^{2}}\left(\partial_{t}+u \partial_{x}\right)^{2} p-\Delta p+\frac{\nu_{\mathrm{t}}}{c^{2}}\left(\partial_{t}+u \partial_{x}\right) p=0
$$

An der Wand müssen Haftbedingung und die Impedanzbeziehung zwischen Vertikalschnelle und Wechseldruck erfüllt werden ${ }^{7}$ :

\footnotetext{
${ }^{7}$ In Gleichung (3.57) ändert sich wieder gegenüber (3.51) das Vorzeichen, weil eine positive Radialschnelle in die Wand hineinzeigt, während im Falle der ebenen Wand eine positive $y$-Komponente der Schnelle aus der Wand heraus zeigt.
} 


$$
\begin{array}{lll}
v_{x}=0 & , r=R \\
v_{r}=+\frac{\omega^{\prime}}{\omega} \frac{p}{Z(\omega)} & , r=R
\end{array}
$$

Das Paar (3.53), (3.54) von Differentialgleichungen hat wieder zwei Klassen wellenartiger Lösungen. Die STOKES-Moden erfüllen

$$
p \equiv 0
$$

wodurch das Schnellefeld $\left(k_{\mathrm{St}}\right.$ wie in $\left.(3.43)\right)$ die folgende Gestalt hat:

$$
\begin{aligned}
& v_{x}=\quad v_{\mathrm{St}}^{0} J_{0}\left(k_{\mathrm{St}} r\right) \cdot e^{-i \omega t+i k_{x} x} \\
& v_{r}=-\frac{i k_{x}}{k_{\mathrm{St}}} v_{\mathrm{St}}^{0} J_{1}\left(k_{\mathrm{St}} r\right) \cdot e^{-i \omega t+i k_{x} x}
\end{aligned}
$$

Die zweite Klasse von Lösungen hat ein Wechseldruckfeld

$$
p=p_{0} J_{0}\left(k_{r} r\right) \cdot e^{-i \omega t+i k_{x} x}
$$

mit der Radialwellenzahl

$$
k_{r}^{ \pm}= \pm i \sqrt{k_{x}^{2}-\frac{\left(\omega-u k_{x}\right)^{2}}{c^{2}+i \nu_{\mathrm{t}}\left(\omega-u k_{x}\right)}}
$$

Einsetzen in die NAVIER-STOKES-Gleichung liefert für diese Teillösungen das Schnellefeld

$$
\begin{aligned}
v_{x} & =\frac{k_{x} p_{0}}{\rho\left(\omega-u k_{x}+i \nu_{\mathrm{t}}\left(k_{x}^{2}+k_{r}^{2}\right)\right)} J_{0}\left(k_{r} r\right) \cdot e^{-i \omega t+i k_{x} x} \\
v_{r} & =\frac{-k_{y} p_{0}}{\rho\left(\omega-u k_{x}+i \nu_{\mathrm{t}}\left(k_{x}^{2}+k_{r}^{2}\right)\right)} J_{1}\left(k_{r} r\right) \cdot e^{-i \omega t+i k_{x} x}
\end{aligned}
$$

Wiederum legen Haft- und Impedanzbedingung die relative Amplitude beider Teilwellen fest und führen auf die Dispersionsbeziehung 


$$
\begin{aligned}
1+\left[\left(i\left(1-\frac{u k_{x}}{\omega}\right)^{2}-\frac{\nu_{\mathrm{t}}}{\omega}\left(k_{x}^{2}+k_{r}^{2}\right)\right.\right. & \left.\left(1-\frac{u k_{x}}{\omega}\right)\right) \frac{\rho \omega}{k_{r} Z} \\
+ & \left.\frac{k_{x}^{2}}{k_{\mathrm{St}} k_{r}} \frac{J_{1}\left(k_{\mathrm{St}} R\right)}{J_{0}\left(k_{\mathrm{St}} R\right)}\right] \frac{J_{0}\left(k_{r} R\right)}{J_{1}\left(k_{r} R\right)}=0
\end{aligned}
$$

Rechnen ohne Kompressibilität liefert eine Gleichung, in der der Term proportional zu $k_{x}^{2}+k_{r}^{2}$ fehlt. Für verschwindende Zähigkeit fehlt neben dem eben genannten Term auch der zweite Summand in der eckigen Klammer und (3.65) geht in die schon bekannte Beziehung (3.27) mit $m=0$ über.

\subsubsection{Verlauf der Moden für kompressible Rechnung im Rohr}

Die Dispersionsbeziehung (3.65) führt zu einer nur leicht veränderten Abhängigkeit der Ausbreitungswellenzahlen im nachgiebigen Rohr, wie Abbildung 3.19 zeigt. Wieder ist die instabile Mode erst ab einer Grenzfrequenz unterhalb von $f_{0}$ räumlich angefacht.

Ähnlich wie in Abschnitt 3.3.2 verschiebt sich diese Grenzfrequenz für größere turbulente Zähigkeiten weiter in Richtung der Kammerresonanz, während die räumliche Dämpfung unterhalb zunimmt. Der Abfall des Realteils der Wellenzahl wird ebenso flacher, steigt allerdings (keine Abbildung) für sehr große Werte von $\nu_{\mathrm{t}}$ bei hohen Frequenzen wieder an.

Die übrigen Moden im nachgiebigen Rohr sind-wie zu erwarten-stärker gedämpft als bei verlustfreier Rechnung. Ansonsten sind die Unterschiede eher gering.

\subsubsection{Zusammenfassung}

Die Berücksichtigung einer empirischen „turbulenten“ Zähigkeit führt zu den folgenden Ergebnissen:

- Der Verlauf der Wellenzahlen der Moden im nachgiebigen Rohr verändert sich nur unwesentlich. Im allgemeinen sind die Moden stärker gedämpft.

- Die Instabilitätsmode ist für niedrige Frequenzen ebenfalls gedämpft und weist erst ab einer Grenzfrequenz, die sich mit wachsendem $\nu_{\mathrm{t}}$ der Kammerresonanz nähert, eine räumliche Anfachung auf. 

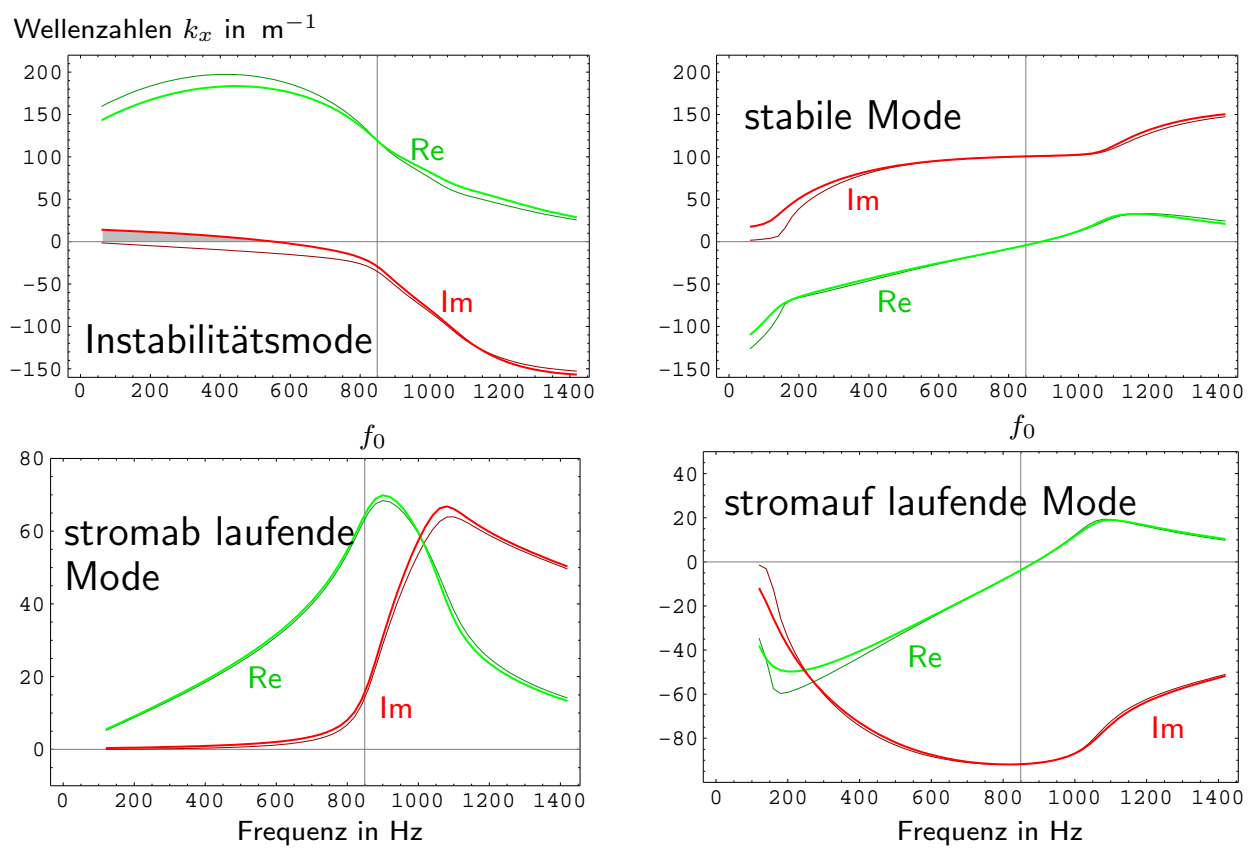

Abbildung 3.19: Vergleich der Lösungen im Rohr unter Berücksichtigung der Kompressibilität und einer Zähigkeit von $\nu_{\mathrm{t}}=300 \nu$ (dicke Linien) mit den herkömmlichen Lösungen (dünne Linien) bei $u=60 \mathrm{~m} \cdot \mathrm{s}^{-1}$

- Für physikalisch sinnvolle Werte von $\nu_{\mathrm{t}}$ ist nicht zu erkennen, daß die Anfachung bei hohen Frequenzen zusammenbricht.

\subsection{Einfache Modelle-Resultate}

In diesem Kapitel habe ich einfache Modelle der Strömungsakustik bei lokal nachgiebigen Kanalwänden untersucht.

In jedem Fall existiert eine instabile hydrodynamische Mode, deren räumliche Anfachung jenseits der Resonanzfrequenz große Werte erreicht. Diese Anfachung bleibt groß auch für hohe Frequenzen.

Es scheint, als sind die Modelle nicht mächtig genug, das experimentell beobachtete Verstärkungsverhalten abzubilden. Es gibt dafür mindestens vier mögliche Ursachen:

1. Für den tatsächlichen Verlauf ist das Gleichströmungsprofil nahe der Wand 
und gegebenenfalls die Ortsabhängigkeit (Wandabstand) der turbulenten Zähigkeit wesentlich.

2. Die endliche Breite der Kammern könnte die Näherung lokaler Nachgiebigkeit bei hohen Frequenzen zusammenbrechen lassen, so daß eine Rechnung mit Kassettierung der Wand notwendig würde.

3. Die räumliche Anfachung der Instabilitätsmode macht sich nicht eins zu eins bemerkbar, weil vielleicht die Anregung der Mode durch den eingestrahlten (am Stromauf-Ende des Meßobjektes) oder die Abstrahlung des Schalls (am Stromab-Ende) stark von der Frequenz (und dar Machzahl) abhängt.

4. Das Verstärkungsphänomen könnte sich grundsätzlich einer linearen Behandlung entziehen, wenn etwa die räumliche Deformation des Gleichströmungsprofils durch den Energietransfer in die Instabilität entscheidend ist.

Punkt 2 und Punkt 4 werde ich in dieser Arbeit nicht weiter untersuchen; allerdings deuten hier nicht vorgestellte Rechnungen darauf hin, daß der Fall 2 für eine ebene Wand nicht eintritt, sofern dem Experiment entsprechende Abmessungen gewählt werden.

Den Punkten 1 und 3 werde ich in den nächsten Kapiteln nachgehen. 


\section{Kapitel 4}

\section{Modelle mit Gleichströmungsprofil}

Eine wesentliche Eigenschaft der instabilen Mode aus dem vorangegangenen Kapitel ist, daß sie offenbar für beliebig hohe Frequenzen räumlich angefacht ist. Obwohl man von der räumlichen Anfachungsrate nicht direkt auf den Transmissionsfaktor $T \rightarrow$ schließen kann, ist nicht einzusehen, warum es für hohe Frequenzen nach wie vor eine instabile Mode gibt, sich diese aber überhaupt nicht mehr bemerkbar macht.

Es liegt daher nahe, den Einfluß eines realistischen Strömungsprofils auf den Modenverlauf zu untersuchen.

Als ähnliches Beispiel habe ich die KELvin-HeLmHoltz-Instabilität vor Augen. Modelliert man die Scherschicht lediglich mit einem Stufenprofil, so ist die Mode auch für beliebig hohe Frequenzen noch räumlich angefacht. Wählt man nur die nächstbeste Näherung, nämlich ein Profil mit endlicher Scherschichtbreite und linear anwachsender Gleichströmung in dieser Schicht, so schrumpft der Verstärkungsbereich auf ein endlich breites Frequenzband, das zumindest in der Größenordnung die tatsächlichen Verhältnisse richtig wiedergibt.

Ich beschränke mich bei der Untersuchung auf ein kompressibel durchströmtes Rohr, da andere Geometrien oder Vernachlässigen der Kompressibilität die Rechnung nicht wesentlich vereinfachen und der Einfluß dieser Faktoren bereits in den vorhergehenden Abschnitten eingehend untersucht wurde. 


\subsection{Die Differentialgleichung für die kompressible Rohr- strömung}

Weil $u$ nun eine von $r$ abhängige Größe ist,

$$
\mathbf{u}(\mathbf{x})=u(r) \mathbf{e}_{x}
$$

taucht in den linearisierten EuLER-Gleichungen ein zusätzlicher Term auf, während die Kontinuitätsgleichung unverändert bleibt,

$$
\begin{aligned}
& \left(\partial_{t}+u \partial_{x}\right) v_{x}+\partial_{r} u v_{r}=-\frac{1}{\rho} \partial_{x} p \\
& \left(\partial_{t}+u \partial_{x}\right) v_{r}=-\frac{1}{\rho} \partial_{r} p \\
& \left(\partial_{t}+u \partial_{x}\right) p+\rho c^{2}\left(\partial_{x} v_{x}+\partial_{r} v_{r}+\frac{1}{r} v_{r}\right)=0
\end{aligned}
$$

Wie in den vorherigen Rechnungen sollen die zu betrachtenden Lösungen zeitlich und räumlich (in x-Richtung) harmonisch sein.

Addiert man die $x$-Ableitung von Gleichung (4.2) und die $\left(\partial_{r}+\frac{1}{r}\right)$-Ableitung von Gleichung (4.3), so kann man mit Hilfe von Gleichung (4.4) und nochmals (4.3) die Wechselgeschwindigkeitskomponenten aus der Summe eliminieren und erhält folgende gewöhnliche Differentialgleichung für $p$ :

$$
\left(\partial_{r}^{2}+\frac{1}{r} \partial_{r}-k_{x}^{2}+\frac{1}{c^{2}}\left(\omega-u k_{x}\right)^{2}+\frac{2 k_{x} \partial_{r} u}{\omega-u k_{x}} \partial_{r}\right) p=0
$$

Gleichung (4.5) beschreibt das Wechseldruckprofil in Abhängigkeit vom Gleichströmungsprofil.

\subsection{Wahl der Randbedingungen}

Da Gleichung (4.5) zweiten Grades ist, sind drei Randbedingungen notwendig, um das Spektrum der möglichen Ausbreitungswellenzahlen - in Abhängigkeit von Frequenz, Machzahl und Gleichströmungsprofil-zu ermitteln. 
Da ich mich auf die rotationssymmetrischen Lösungen beschränke, muß in der Mitte der Strömung gelten $(p=p(r))$ :

$$
\partial_{r} p(0)=0
$$

Die Amplitude der Lösung setze ich willkürlich so, daß in der Mitte der Wert

$$
p(0)=\text { const. } \neq 0
$$

erreicht wird. Der Fall verschwindenden Drucks bei $r=0$ muß nicht gesondert verfolgt werden, da er mit Randbedingung (4.6) nur auf die Lösung $p \equiv 0$ führt. An der nachgiebigen Wand muß die Impedanzbeziehung erfüllt werden. Ausgedrückt ausschließlich durch den Wechseldruck lautet sie

$$
\partial_{r} p(R)=i\left(1-\frac{u(R) k_{x}}{\omega}\right)^{2} \frac{\rho \omega}{Z} p(R)
$$

Dabei wird offengelassen, ob $u$ bei $r=R$ verschwindet.

\subsection{Wahl des Grundströmungsprofils}

Die Wahl des Gleichströmungsprofils ist insofern schwierig, als uns gesicherte Erkenntnisse über Details der Gleichströmung vor der Kassettierung nicht vorliegen. In Vorgängerarbeiten [17] hat sich allerdings herausgestellt, daß ein modifiziertes Logarithmus-Profil,

$$
u(r)=u_{\epsilon}+\frac{u_{\tau}}{\kappa} \ln \left(1+\frac{R-r}{y_{0}}\right)
$$

in Verbindung mit einer durch Schubspannungseffekte modifizierten Wandnachgiebigkeit die Verhältnisse offenbar qualitativ gut wiedergeben kann. Ich werde dieses Profil daher weiterbenutzen. Zur Motivation dieser Wahl verweise ich auf die genannte Arbeit. Für die numerische Auswertung wähle ich wie in [17]

$$
\begin{aligned}
& u_{\epsilon}=2,3 u_{\tau} \\
& y_{0}=0,017 R
\end{aligned}
$$

Es sei darauf hingewiesen, daß bei diesem Profil die Gleichströmungsgeschwindigkeit an der Wand nicht verschwindet, sondern den endlichen Wert $u_{\epsilon}$ annimmt. 
Die über den Querschnitt gemittelte Gleichströmungsgeschwindigkeit

$$
\bar{u}=\frac{1}{\pi R^{2}} \int_{0}^{2 \pi} d \phi \int_{0}^{R} d r r u(r)=\frac{2}{R^{2}} \int_{0}^{R} d r r u(r)
$$

stellt den Zusammenhang zwischen der Schubspannungsgeschwindigkeit $u_{\tau}$ und der Gleichströmungsgeschwindigkeit aus dem vorhergehenden Kapitel her.

\subsection{Zur Lösung der Differentialgleichung}

Um die Differentialgleichung (4.5) mit den Randbedingungen (4.6)-(4.8) zu lösen, empfiehlt sich ein Shooting-Verfahren, bei dem die Integration mit einem Schätzwert $k_{x}^{i}$ für die zu ermittelnde Wellenzahl $k_{x}$ bei $r=0$ begonnen wird und die Abweichung $\delta$ des Randwertes von der geforderten Randbedingung bei $r=R$ dazu benutzt wird, linear auf einen besseren Schätzwert zu approximieren.

In der Nähe einer tatsächlichen Lösung sollte $\delta$ etwa linear von der Differenz zwischen geschätzter und tatsächlicher Wellenzahl abhängen:

$$
\delta^{i} \approx \alpha\left(k_{x}^{i}-k_{x}\right)
$$

Kennt man die Fehler zweier verschiedener Schätzwerte, so läßt sich $\alpha$ näherungsweise bestimmen:

$$
\alpha \approx \frac{\delta^{i}-\delta^{i+1}}{k_{x}^{i}-k_{x}^{i+1}}
$$

Hiermit kann man einen besseren Schätzwert $k_{x}^{i+2}$ für $k_{x}$ ermitteln. Stellt man nämlich Gleichung (4.11) nach $k_{x} \mathrm{um}$, so gilt ebenfalls näherungsweise

$$
k_{x} \approx k_{x}^{i}-\frac{\delta^{i}}{\alpha} \doteq k_{x}^{i+2}
$$

Wie $\delta$ im einzelnen gewählt wird, ist nicht so bedeutsam, sofern numerische Schwierigkeiten nicht auftreten; möglich ist etwa

$$
\delta=\frac{\partial_{r} p(R)}{p(R)} \frac{Z}{i \rho \omega}\left(\frac{\omega}{\omega-u(R) k_{x}}\right)^{2}-1
$$

siehe Randbedingung (4.8).

Als Startwerte bieten sich die Lösungen einfacherer Modelle an. Die resultierenden Moden müssen aber nicht die einzigen Lösungen sein, da das Modell mit 
Strömungsprofil nun mehr Freiheitsgrade besitzt und zusätzliche Lösungen denkbar sind. Diese lassen sich nur finden, indem der Algorithmus mit vielen Startwerten getestet wird.

Abgesehen davon, daß Lösungen übersehen werden können, wenn keiner der Schätzwerte hinreichend nahe liegt, ist auch nicht gesagt, daß der eben beschriebene Algorithmus für alle Lösungen konvergiert.

Für die Integration habe ich die Mathematica-Funktion NDSolve verwendet, mit der gewöhnliche Differentialgleichungen bei Angabe geeigneter Anfangsbedingungen verhältnismäßig einfach gelöst werden können.

\subsubsection{Die instabile Mode}

In Abbildung 4.1 sind einige der Lösungen mit den Moden aus Abschnitt 3.2.2 verglichen, wobei die Bezeichnungen von dort übernommen wurden.

Überraschenderweise zeigt sich, daß die der Instabilitätsmode ähnlichste Lösung nur oberhalb der Resonanzfrequenz zu existieren scheint. Darüber hinaus gibt es mindestens eine weitere Lösung, die für tiefe Frequenzen bis knapp oberhalb der Resonanz existiert und keiner der bekannten Moden ähnelt. Für ein kleines Frequenzintervall koexistieren diese Lösungen — zumindest bei der hier gewählten Strömungsgeschwindigkeit von $60 \mathrm{~m} \cdot \mathrm{s}^{-1}$.

Die zusätzliche Mode ist instabil, was man mit dem Kriterium nach JonEs und MorGAN nachprüfen kann. Dieses ist zwar auf die andere Teillösung bei hohen Frequenzen zunächst nicht anwendbar, tatsächlich gehören aber beide Lösungen für komplexe Frequenzen zu einer mehrdeutigen Funktion, ähnlich etwa dem komplexen Logarithmus. In Abbildung 4.2 ist ein Teil des zugehörigen Graphen zu sehen. Wie man sieht, gehören beiden Teilmoden zu einer Zusammenhangskomponente. Weitere Details des Verlaufes nahe der Resonanzfrequenz (die von dem Streifen umschlossen wird) sind noch nicht untersucht.

Für das eigentümliche Verhalten der Lösungen, bei $\operatorname{Im} k_{x}=0$ nicht mehr zu existieren, gibt es wieder eine zumindest mathematisch einfache Erklärung (siehe auch Abschnitt 3.2.5): Das Modell läßt nämlich reelle Ausbreitungswellenzahlen gar nicht zu. Wäre $k_{x}$ reell, so wären es auch die Differentialgleichung (4.5) und die Randbedingungen bei $r=0$, da der Wechseldruck dort ohne Beschränkung der Allgemeinheit reell gewählt werden darf. Nach der Integration der Differentialgleichung bis $r=R$ wären dann aber auch der Wechseldruck und seine $r$-Ableitung bei $r=R$ reell. Die Randbedingung (4.8) ließe sich so unmöglich erfüllen, da die Wandimpedanz einen nicht verschwindenden Realteil besitzt.

Die physikalische Interpretation dieser Befunde ist allerdings schwieriger. Ohne 
Wellenzahlen $k_{x}$ in $\mathrm{m}^{-1}$
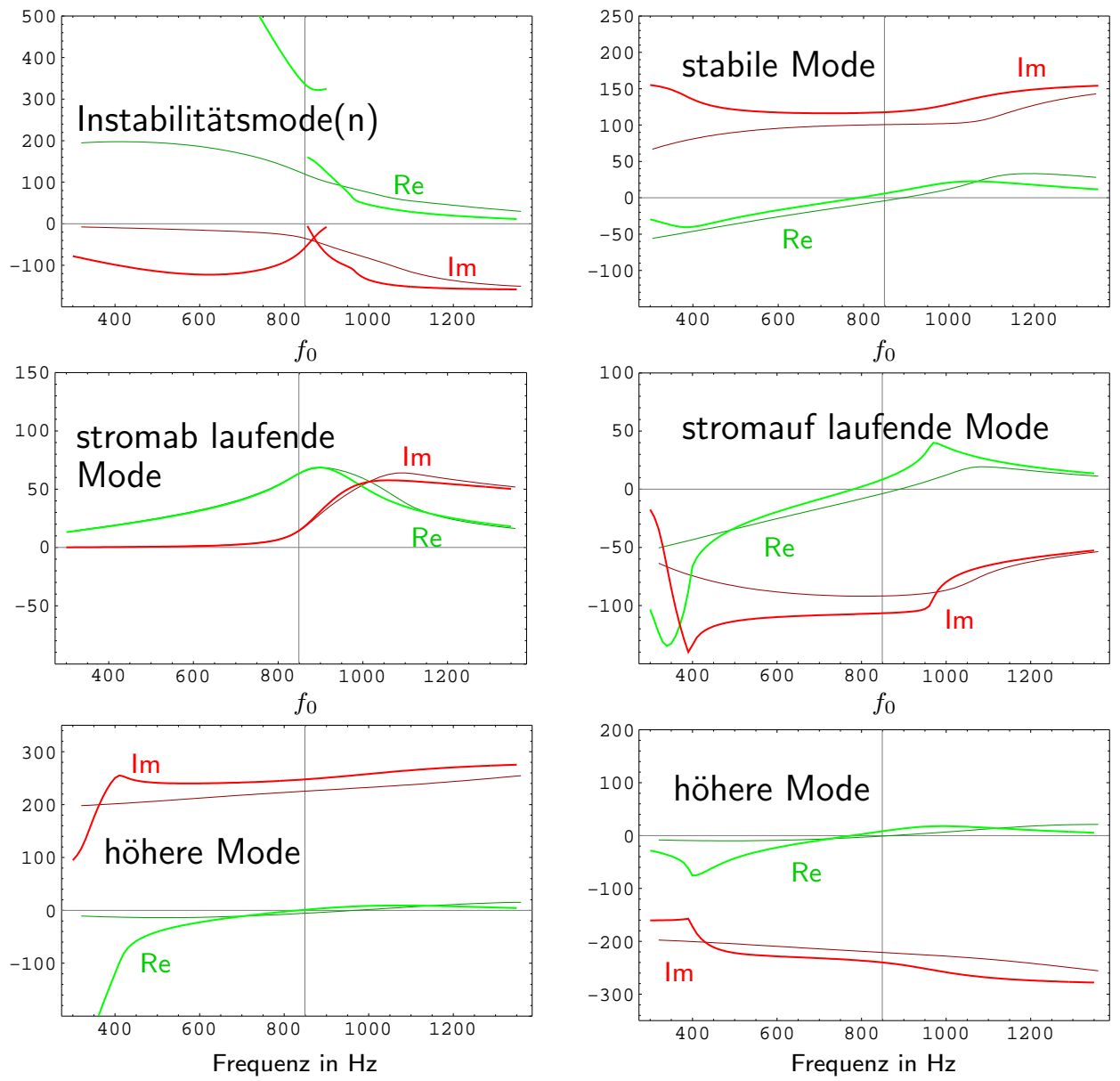

Abbildung 4.1: Vergleich der Lösungen im Rohr mit Rechteckprofil (dünne Linien) und dem logarithmischen Profil (dicke Linien) bei $\bar{u}=60 \mathrm{~m} \cdot \mathrm{s}^{-1}$ 

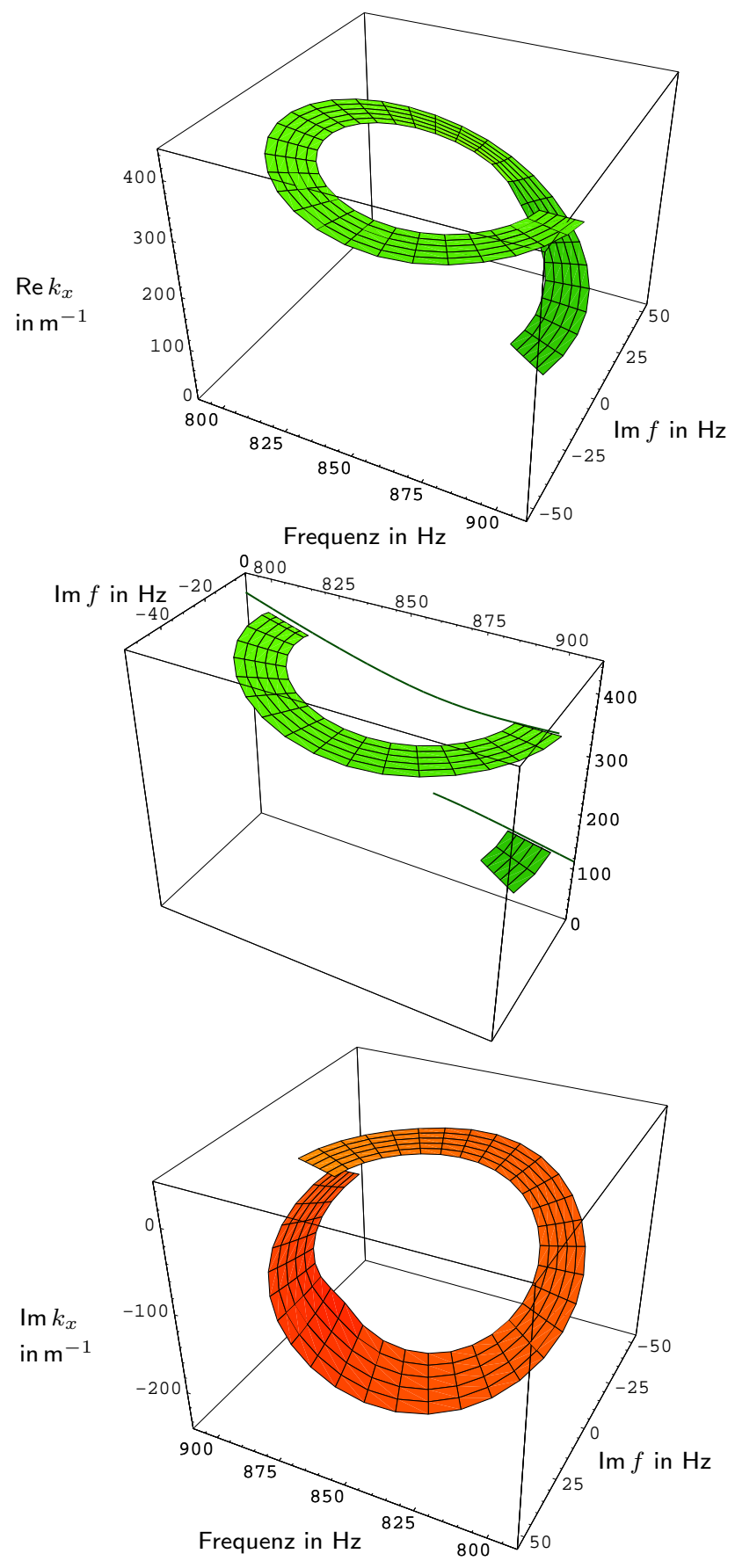

Abbildung 4.2: Wellenzahlen der Instabilitätsmode für komplexe Frequenzen $f$, $34 \mathrm{~Hz}<\left|f-f_{0}\right|<49 \mathrm{~Hz}$ und $\arg \left(f-f_{0}\right)=-0,18 \pi \ldots 1,92 \pi$ bei $\bar{u}=60 \mathrm{~m} \cdot \mathrm{s}^{-1}$. 
Gelegenheit, dieses Modell noch eingehender untersuchen zu können, scheint mir das Problem in der Projektion der physikalischen Lösungen auf den Raum der zeitlich und in $x$-Richtung harmonischen Funktionen zu liegen. Vielleicht ist die Zerlegung in derartige Funktionen mit diesem Randwertproblem nicht wirklich verträglich.

In diesem Zusammenhang wäre es interessant zu wissen, ob es bei den anderen Moden auch Mehrdeutigkeiten gibt. Ich habe zunächst keine weiteren gefunden.

\subsubsection{Die anderen Moden}

Das Verhalten der anderen Lösungen entspricht mehr oder weniger dem einfachen Modell aus Abschnitt 3.2.2. Lediglich bei einigen kritischen MACH-Zahlen und Frequenzen gibt es große Abweichungen: Hier kreuzen sich Moden, siehe Abbildung 4.1 mit der stromauf laufenden und der darunter abgebildeten höheren Mode bei etwa $400 \mathrm{~Hz}$. Sieht man von dem eigentümlichen Verhalten der instabilen Mode einmal ab, scheint die Näherung durch ein Rechteckprofil durchaus gerechtfertigt zu sein.

\subsection{Wechseldruckprofile}

In den einfacheren Modellen war die Ortsabhängigkeit der Lösungen explizit bekannt. Ich habe daher auf eine Darstellung etwa der Druckprofile (in Abhängigkeit vom Wandabstand) verzichtet.

Weil die Wechselfelder hier nur noch numerisch ermittelt werden können, sind in Abbildung 4.3 die Beträge des Wechseldruckes für die einzelnen Moden aufgetragen. Die Frequenz von $900 \mathrm{~Hz}$ und die schon öfter gewählte, querschnittsgemittelte Grundströmungsgeschwindigkeit von $60 \mathrm{~m} \cdot \mathrm{s}^{-1}$ sind zwar willkürlich gewählt, liegen aber in dem Parameterbereich, in dem das Meßobjekt den Schall verstärkt. Sie sollten daher typisch sein.

Auch hier zeigt sich, daß die Abweichungen beim Übergang vom Rechteck- zum realistischeren Logarithmus-Profil nicht besonders groß sind.

Die Grundmoden füllen wie erwartet mehr oder weniger das ganze Rohrinnere aus, während die höheren Moden die typischen Knotenflächen zeigen. Wie in den vorhergegangenen Modellen fällt die jenseits der Resonanzfrequenz existierende instabile Teilmode zur Kanalmitte hin nur moderat ab.

Hiervon unterscheidet sich die unterhalb der Resonanzfrequenz hinzugekommene Teillösung ganz erheblich. In Abbildung 4.4 ist sie im Vergleich zu der ursprüng- 

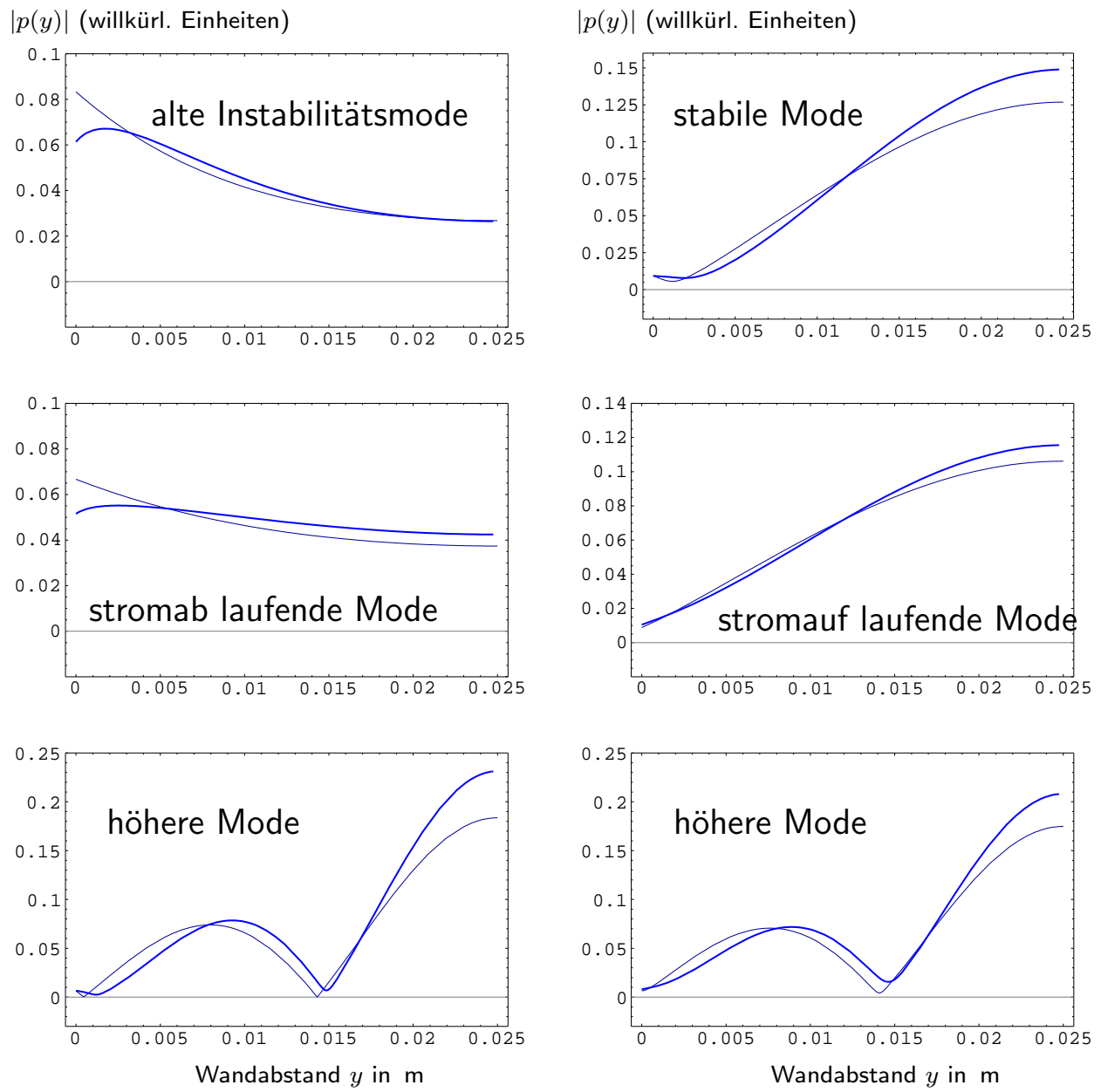

Abbildung 4.3: Wechseldruckamplitude bei $\bar{u}=60 \mathrm{~m} \cdot \mathrm{s}^{-1}$ und $900 \mathrm{~Hz}$ in Abhängigkeit vom Wandabstand. Rechnung mit Logarithmusprofil (dicke Linien) und Rechteckprofil (dünne Linien) 

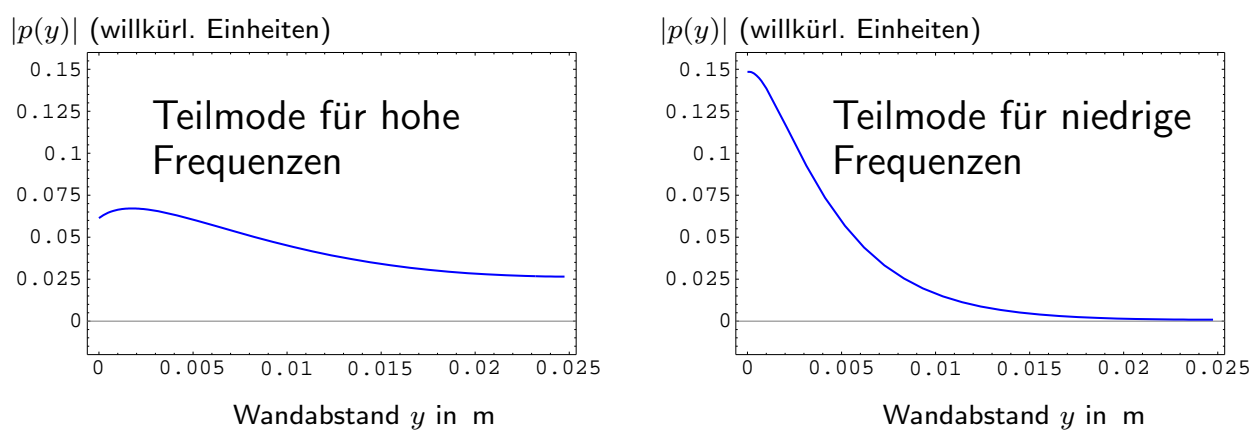

Abbildung 4.4: Wechseldruckamplitude bei $\bar{u}=60 \mathrm{~m} \cdot \mathrm{s}^{-1}$ und $900 \mathrm{~Hz}$ in Abhängigkeit vom Wandabstand

lichen Lösung zu sehen. Moden mit dieser Wellenzahl sind viel stärker an der Wand lokalisiert. Der Realteil von $k_{x}$ ist hier sehr groß, was in inkompressibler Näherung mit kleinen Eindringtiefen einhergeht, sofern eine Mode überhaupt an der Wand lokalisiert ist ${ }^{1}$.

Interessanterweise ähneln sich die Profile der stromab laufende Grundmode genannten Lösung und der Instabilität, so daß man geneigt ist, bei den hier gewählten Strömungsparametern dieses Paar als die hinzugekommenen hydrodynamischen Moden anzusehen. Für andere Frequenzen und Strömungsgeschwindigkeiten könnte diese Identifizierung allerdings auch wieder anders aussehen, worauf ich in Abschnitt 3.2.4 bereits eingegangen bin.

\subsection{Zusammenfassung}

Auch dieses Modell liefert keine Instabilitätsmode, die so verläuft, wie wir es nach den experimentellen Ergebnissen erwartet hätten. Nichts deutet darauf hin, daß durch Wahl eines realistischen Strömungsprofils die Anfachung bei höheren Frequenzen zusammenbricht. Der allmähliche Abfall der Grundströmung auf Null in Wandnähe ist also nicht der Mechanismus, der zu einem endlich breiten Verstärkungsband führt.

Die eigentümliche Aufspaltung der instabilen Mode in mindestens zwei, nur in begrenzten Frequenzintervallen existierende Teillösungen verdient eine genauere Untersuchung, insbesondere die Fragen, inwiefern diese Erscheinung von der Form des Gleichströmungsprofils abhängt und ob es Ansätze gibt, die mehrdeutige

\footnotetext{
${ }^{1}$ Für einfache Modelle und inkompressible Rechnung gilt $\operatorname{Re} k_{x}= \pm \operatorname{Im} k_{y}$.
} 
Funktionen $k_{x}(\omega)$ vermeiden.

Anstatt die Modelle noch komplexer zu machen, etwa durch Modellierung turbulenter Schubspannungen oder Wirbelviskosität, ist es näherliegend, zunächst die Resultate zu testen, die die Spektren einfacher Modelle in einer Streurechnung liefern. Die bloße Existenz einer instabilen Lösung muß noch nicht zu einer Schallverstärkung führen, nämlich dann nicht, wenn die Präparation des Experimentes die Anregung dieser Mode gar nicht zuläßt oder erschwert. 


\section{Kapitel 5}

\section{Streuung}

In diesem Kapitel werde ich die Lösungen aus den Abschnitten 3.2.2 und 3.2.3 benutzen, um die Transmissions- und Reflexionsfaktoren zu berechnen, die BRANDES in [3] präsentiert.

Die Streurechnungen - mit Hilfe der sogenannten WIENER-HopF-Methode - sind sehr aufwendig. Darüber hinaus ist die benutzte Methode zwar in der theoretischen Strömungsakustik, aber nicht unbedingt unter Experimentatoren geläufig, weswegen ich das Prinzip in Abschnitt 5.1 erläutern werde.

In Abschnitt 5.2 stelle ich das von KocH und MöHRING untersuchte Problem vor, ohne allerdings auf die Formeln im einzelnen eingehen zu können. Ich muß für diesen Teil die Kenntnis der Arbeit [10] voraussetzen. Anschließend beschreibe ich die Vorhersagen, die diese Rechnung für die Streufaktoren bei den uns interessierenden Parametern macht.

Um Inkonsistenzen mit den zitierten Ergebnissen zu vermeiden, gilt für dieses Kapitel abweichend von den vorherigen die Konvention, daß Wechselgrößen proportional zu

$$
e^{i \omega t+i \mathbf{k} \mathbf{x}}
$$

sind, wobei für nach rechts laufende Wellen $k_{x}$ dann negativ ist.

In Abschnitt 5.3 werde ich die Ergebnisse für einen Kanal mit kreisförmigem Querschnitt vorstellen. Die Kenntnis von [10] wird auch hier vorausgesetzt. Aus Gründen der Lesbarkeit finden sich die dazugehörigen modifizierten Formeln in Anhang C. 


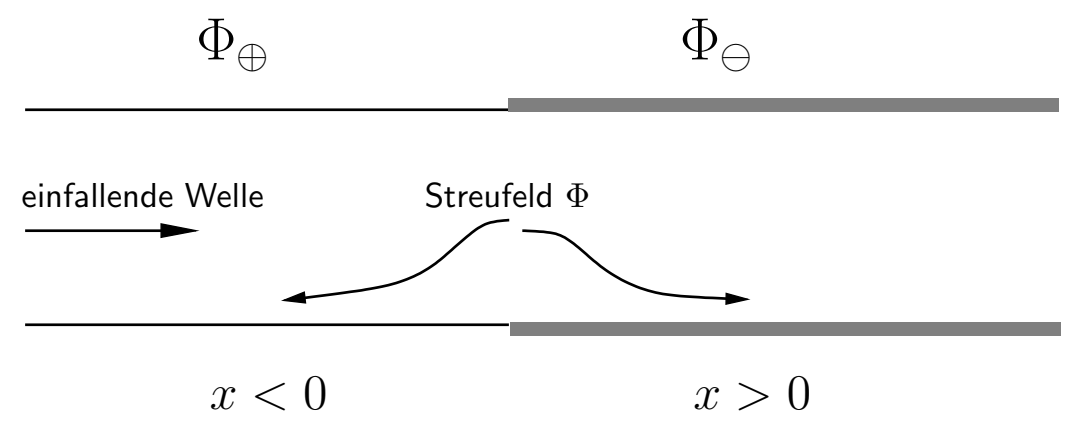

Abbildung 5.1: Ein stückweise homogenes Randwertproblem

\subsection{Die Wiener-Hopf-Methode}

Die Wiener-Hopf-Methode ist ein Verfahren, um stückweise homogene Randwertprobleme zu lösen. Im hier vorliegenden Fall ist das ein Luftkanal, dessen ansonsten schallharte Wand auf einem Stück endlicher Länge resonanzartig nachgibt. Die Randbedingungen dieses Problems weisen also zwei Unstetigkeiten auf, und das Schallfeld muß in den drei Abschnitten aus unterschiedlichen Moden kombiniert werden.

Die WiEnER-HopF-Methode benutzt funktionentheoretische Methoden, um abhängig von einer Teilamplitude („einfallende Welle“) das gesamte Wechselfeld (,gestreute Wellen“) zu berechnen.

Eine genauere Beschreibung dieser Methode und ihrer Anwendungen findet man in $[16]$.

\subsubsection{Der abstrakte Lösungsweg}

Gegeben ist ein lineares Randwertproblem für die Größe $\Phi$ mit einer Koordinate $x \in \mathbb{R}$. Die Randbedingungen sind entlang dieser Koordinate homogen bis auf - der Einfachheit halber - einen Sprung bei $x=0$ (siehe Abb. 5.1).

Statt der FouRIER-Transformierten werden nun die halbseitigen FouRIERTransformierten 


$$
\begin{aligned}
& \Phi_{\oplus}(k)=\int_{-\infty}^{0} d x \Phi(x) e^{-i k x} \\
& \Phi_{\ominus}(k)=\int_{0}^{+\infty} d x \Phi(x) e^{-i k x}
\end{aligned}
$$

eingeführt, deren Summe die Transformierte von $\Phi$ ist. Die Wellenzahlen $k$ können komplex sein.

Der erste entscheidende Schritt ist die Annahme, daß durch Dämpfungs- oder vergleichbare Effekte $\Phi$ im Unendlichen mit einer räumlichen Dämpfungskonstante $\epsilon$ exponentiell abfällt. Das ist physikalisch sicherlich immer richtig, auch wenn im Modell vielleicht gar keine Dämpfungseffekte berücksichtigt werden und $\epsilon$ nur eine infinitesimale mathematische Größe ist.

Bei Streurechnungen, einer wichtigen Anwendung dieser Methode, macht die einfallende Welle dieses Abklingverhalten zunichte, denn sie kommt ja gerade aus dem Unendlichen. Bei diesen Rechnungen wird der WiEnER-HopF-Formalismus auf das Streufeld angewandt, also dem Feld, das übrigbleibt, wenn man vom Gesamtfeld die einfallende Welle abzieht. Für dieses Streufeld ist die Abklingbedingung dann wieder erfüllt.

Durch die Dämpfungskonstante besitzen nun die Transformierten $\Phi_{\oplus}, \Phi_{\ominus}$ sich überschneidende Definitionsbereiche: $\Phi_{\oplus}$ ist wohldefiniert durch Gleichung (5.1) und eine holomorphe Funktion von $k$ in einer oberen Halbebene

$$
S_{\oplus}=\{k \in \mathbb{C}: \operatorname{Im} k>-\epsilon\}
$$

Analog ist $\Phi_{\ominus}$ holomorph in einer unteren Halbebene

$$
S_{\ominus}=\{k \in \mathbb{C}: \operatorname{Im} k<+\epsilon\}
$$

die mit der oberen in einem $2 \epsilon$ breiten Streifen um die reelle Achse überlappt. Das wird später noch wichtig sein.

Um das Problem zu lösen, müssen $\Phi_{\oplus}$ und $\Phi_{\ominus}$ berechnet werden. Dazu muß ich auch die Differentialgleichungen und Randbedingungen auf den jeweiligen Halbachsen transformieren. Das Resultat sind dann Gleichungen, in denen unter anderem $\Phi_{\oplus}(k), \Phi_{\ominus}(k)$ und Randterme des gesuchten Feldes am Sprung auftauchen. Wenn in einer Randbedingung etwa die $x$-Ableitung des Feldes auftaucht, so wird diese nach partieller Integration zu 


$$
\int_{-\infty}^{0} d x \partial_{x} \Phi(x) e^{-i k x}=\Phi\left(0^{-}\right)+i k \Phi_{\oplus}(k)
$$

Über diese Randterme selbst weiß ich in der Regel nichts, so daß ich die Gleichungen so kombinieren muß, damit sie herausfallen oder Kombinationen ergeben, die ich kenne. Wie das im einzelnen zu erreichen ist, hängt von der Art des Randwertproblems und ganz entscheidend von den geforderten Regularitätsbedingungen des unbekannten Feldes bei $x=0$ ab. Vorstellbar ist, daß das gesuchte Feld dort stetig ist, endlich bleibt oder eine KUTTA-Bedingung erfüllt werden soll.

Das Resultat dieser Umformungen ist schließlich eine sogenannte WIENER-HopFGleichung mit der allgemeinen Gestalt

$$
\mathcal{L}(k) \Phi_{\oplus}(k)+\Phi_{\ominus}(k)=m(k)
$$

welche die Funktionen $\Phi_{\oplus}(k)$ und $\Phi_{\ominus}(k)$ verknüpft, aber nur auf dem Überlappungsbereich der Holomorphiegebiete, $S_{\oplus} \cap S_{\ominus}$, gültig ist. Man sieht, wofür eine kleine Dämpfungskonstante eingeführt werden mußte: Ohne diese gäbe es nämlich kein Überlappungsgebiet, und die Gleichung (5.3) würde nirgendwo gelten.

Nun zum entscheidenden Schritt im WiEnER-Hopf-Formalismus:

Ich habe für zwei unbekannte Funktionen $\Phi_{\oplus}, \Phi_{\ominus}$ nur eine Bestimmungsgleichung. Angenommen, ich könnte die Funktionen $\mathcal{L}$ und $m$ so zerlegen, daß auf der einen Seite der Gleichung in der oberen Halbebene holomorphe Funktionen (sogenannte Plus-Funktionen wie $\Phi_{\oplus}$ ) stehen, und auf der anderen Funktionen, die in der unteren holomorph sind (Minus-Funktionen wie $\Phi_{\ominus}$ ), dann erhielte ich ${ }^{1}$ :

$$
\begin{aligned}
\mathcal{L} & \doteq \mathcal{L}_{\oplus} \cdot \mathcal{L}_{\ominus} \\
\mathcal{L}_{\oplus} \Phi_{\oplus}+\frac{\Phi_{\ominus}}{\mathcal{L}_{\ominus}} & =\frac{m}{\mathcal{L}_{\ominus}} \doteq M_{\oplus}+M_{\ominus} \\
\mathcal{L}_{\oplus} \Phi_{\oplus}-M_{\oplus} & =M_{\ominus}-\frac{\Phi_{\ominus}}{\mathcal{L}_{\ominus}} \doteq J(k)
\end{aligned}
$$

Der Sinn dieser Separation ist, daß funktionentheoretische Sätze nun die Existenz einer auf ganz $\mathbb{C}$ holomorphen Funktion, also einer komplexen Potenzrei-

\footnotetext{
${ }^{1}$ Wenn $\mathcal{L}_{\ominus}$ in der unteren Halbebene auch noch nullstellenfrei ist, dann ist auch $1 / \mathcal{L}_{\ominus}$ dort holomorph. Aus diesem Grunde bezeichnet man als Minus- bzw. Plus-Funktionen strenggenommen nur diejenigen, die holomorph und nullstellenfrei sind, damit Quotienten wie in Gleichung (5.4) ebenfalls keine Pole enthalten.
} 
he $J$ sicherstellen. Anders formuliert: Die Ausdrücke auf den beiden Seiten der Gleichungen sind in Wahrheit Kombinationen, die überall holomorph sind.

Der dritte entscheidende Schritt ist die Analyse des Verhaltens der Funktionen $\mathcal{L}_{\oplus}, \mathcal{L}_{\ominus}, M_{ \pm}$und $\Phi_{ \pm}$für $|k| \rightarrow \infty$. Während die ersten beiden Funktionspaare explizit gegeben sind, kann das asymptotische Verhalten der gesuchten Feldtransformierten aus den halbachsentransformierten Randbedingungen gewonnen werden.

Das Verhalten von $J$, welches sich hieraus ergibt, wird in den meisten Fällen abklingend (oder zumindest asymptotisch konstant) sein, und weil $J$ eine Potenzreihe ist, muss sie deswegen identisch Null, seltener gleich einer zunächst unbekannten Konstante sein,

$$
J(k) \equiv 0
$$

Auf diese Weise ist es möglich, aus Gleichung (5.4) zwei Gleichungen

$$
\begin{aligned}
\Phi_{\oplus} & =\frac{M_{\oplus}}{\mathcal{L}_{\oplus}} \\
\Phi_{\ominus} & =M_{\ominus} \mathcal{L}_{\ominus}
\end{aligned}
$$

zu erhalten.

Für die Rücktransformation wird der Residuensatz ausgenutzt:

$$
\Phi(x)=\frac{1}{2 \pi} \int_{-\infty}^{+\infty} d k e^{i k x}\left(\Phi_{\oplus}(k)+\Phi_{\ominus}(k)\right)
$$

Das Ergebnis dieser Rechnung hängt direkt vom Vorzeichen von $x$ ab: Wenn nämlich $x>0$ ist, also nach dem Feld rechts von der Diskontinuität gefragt wird, dann kann man das Integral (5.8) in der oberen komplexen Ebene schließen, weil der Halbkreis im Unendlichen wegen des Exponentialfaktors $\exp (i k x)$ keinen Beitrag liefert. Da $\Phi_{\oplus}$ in der oberen Halbebene holomorph ist, liefert es deswegen auch keinen Beitrag zum Ergebnis, sondern nur die Residuen der Halbachsentransformierten $\Phi_{\ominus}$. Das Feld rechts ist im Ergebnis dann nichts anderes als eine Summe von Moden, deren Wellenzahlen die Pole von $\Phi_{\ominus}$ sind.

Ganz analog setzt sich $\Phi$ für $x<0$ aus Moden zusammen, deren Wellenzahlen Pole von $\Phi_{\oplus}$ sind.

Zusammengefaßt: Die Strategie der WIEnER-Hopf-Methode (und der folgenden Streurechnung) ist es, 

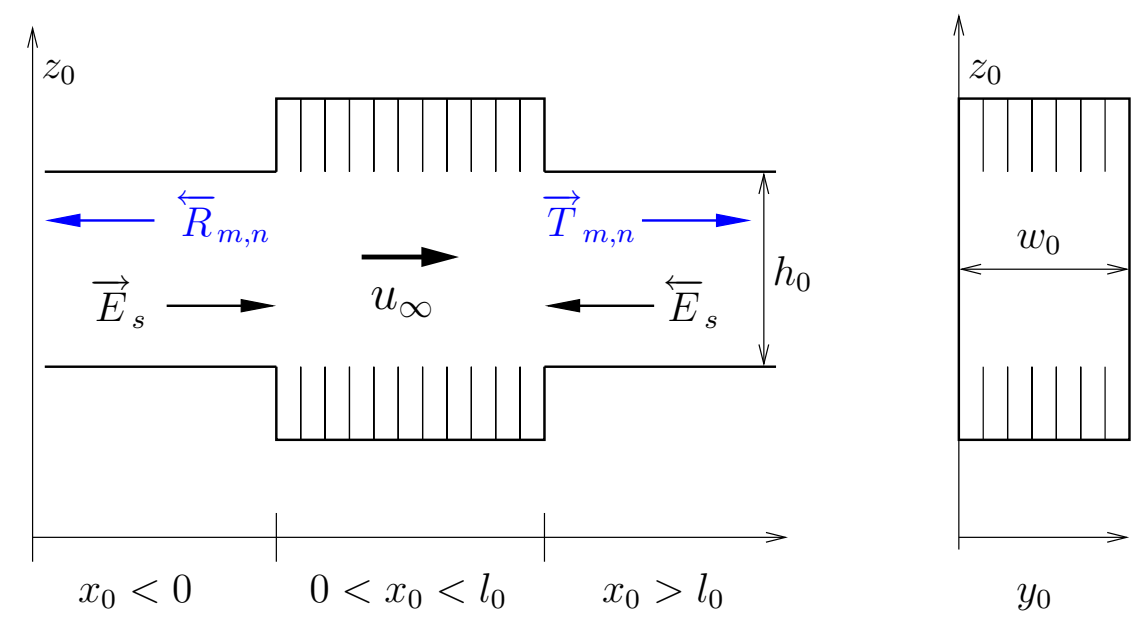

Abbildung 5.2: Von KocH, MöHRING untersuchte Anordnung

1. für das gesuchte Feld links und rechts der Unstetigkeit getrennt zu FouRIERtransformieren,

2. mit Hilfe der Differentialgleichung, der Rand- und Sprungbedingungen eine Gleichung zu formulieren, die als einzige Unbekannten diese Halbachsentransformierten enthält,

3. nach Plus- und Minus-Funktionen zu separieren, deren Definitionsbereiche sich wegen der gedachten kleinen Dämpfung überlappen,

4. das asymptotische Verhalten der entstandenen Kombinationen zu untersuchen, um festlegen zu können, ob diese Kombinationen gleich Null (Regelfall), einer Konstante oder eines höheren Polynoms in $k$ sind, und schließlich

5. die beiden so erhaltenen Gleichungen mit Hilfe des Residuensatzes in den Ortsbereich zu transformieren, um das gesuchte Feld zu ermitteln.

Ein einfaches Beispiel zur Erläuterung der WiEnER-Hopf-Methode findet sich in Anhang B.

\subsection{Die Streurechnung nach KocH und MöHRING}

In [10] wird ein Rechteckkanal untersucht, in dem die Strömungsgeschwindigkeit konstant über den Querschnitt ist. An der Wand fällt diese innerhalb einer unend- 
lich dünn gedachten Wirbelschicht (deren Auslenkung mit $\eta$ bezeichnet wird) auf Null ab. Auf einer Teilstrecke ist der Kanal an zwei gegenüberliegenden Wänden lokal nachgiebig ausgekleidet, siehe Abb. 5.2.

Die Autoren berechnen das Schallfeld und die Streufaktoren ${ }^{2}$ dieses Randwertproblems für zeitlich harmonische Lösungen proportional zu $\exp (i \omega t)$ und diskutieren verschiedene mögliche Sprungbedingungen für die Auslenkung $\eta$ bei $x_{0}=0$, $x_{0}=l_{0}$.

Die Ergebnisse lassen sich mit den experimentellen Daten vergleichen, wenn man als Kanalhöhe $h_{0}$ den Rohrdurchmesser der experimentellen Anordnung wählt und bei den ein- und auslaufenden Wellen die Grundmoden $(m=n=0)$ betrachtet. Von den in der Arbeit unterschiedenen Fällen symmetrischer (s) und asymmetrischer (a) Anregung ist nur der erste für den Vergleich relevant. Ich unterdrücke im folgenden der Übersicht halber die entsprechenden Sub- und Superindizes.

Für die Impedanz der Wand benutze ich (3.7). Die Moden im ausgekleideten Rechteckkanal, die im WiEnER-HopF-Formalismus der Arbeit als Nullstellen der Funktion $\mathcal{L}$ auftreten, sind im Abschnitt 3.2.3 bereits diskutiert worden. Die stromab laufende Instabilitätswelle berücksichtige ich zur Wahrung der Kausalität in den Minusfunktionen (siehe Gln. (26a), (26b) in [10]); KocH und MÖHRING diskutieren allerdings auch die herkömmliche Zerlegung, bei der sich die Instabilitätsmode unter die Stromauf-Moden einreiht. Für den Vergleich der in [10] ermittelten Streufaktoren mit denen aus [3] müssen noch Vorfaktoren und Phasen ergänzt werden, worauf ich in C.5 näher eingehe.

\subsubsection{Wahl der Sprungbedingungen}

Nach der FouRIER-Transformation des gesuchten Feldes tauchen in den Gleichungen die beiden Unbekannten $\eta(0)$ und $\eta(l)$, die Sprünge in der Auslenkung der Wirbelschicht, auf. Deren Werte können nicht aus mathematischen Überlegungen allein abgeleitet werden, weil sich in ihnen die Physik widerspiegelt, die in den Wand-Diskontinuitäten am Ein- und am Auslauf absorbiert wurde. Es ist keinesfalls so, daß eine Wahl mit möglichst hoher Regularität (z. B. Stetigkeit oder Differenzierbarkeit der Auslenkungsfunktion am Einlauf-instationäre KUTTABedingung) ein physikalisch sinnvolles Ergebnis sicherstellt. Durch die sehr singuläre Vereinfachung der tatsächlichen Verhältnisse-Schrumpfen des Gleich-

\footnotetext{
${ }^{2}$ Die Amplituden der ausbreitungsfähigen Moden im stromauf gelegenen Teil des Kanals heißen $\overleftarrow{R}$ stromab $\vec{T}$. Bei Beschallung stromauf sind daher die $\overleftarrow{R}$ Transmissions- und die $\vec{T}$ die Reflexionsfaktoren.
} 


\begin{tabular}{|c|c|c|c|c|c|}
\hline Gl. 1 & Gl. 2 & \multicolumn{4}{|c|}{ Regularität an den Sprüngen } \\
\hline & & $\eta$ bei $x=0$ & $\eta$ bei $x=l$ & $p$ bei $x=0$ & $p$ bei $x=l$ \\
\hline$\eta(0)=0$ & $\eta(l)=0$ & KuTTA & $\infty$ & endlich & $\infty$ \\
\hline$\eta(0)=0$ & Gl. (C.39) & KuTTA & endlich & endlich & endlich \\
\hline$\eta(0)=0$ & Gl. (C.36) & KuTTA & $\infty$ & 0 & $\infty$ \\
\hline$\eta(l)=0$ & Gl. (C.39) & endlich & 0 & endlich & endlich \\
\hline$\eta(l)=0$ & Gl. (C.36) & endlich & $\infty$ & 0 & $\infty$ \\
\hline Gl. (C.36) & Gl. (C.39) & endlich & endlich & 0 & endlich \\
\hline
\end{tabular}

Tabelle 5.1: Mathematisch naheliegende Wahlen für $\eta(0), \eta(l)$

strömungsprofils auf eine unendlich dünne Schicht, diskontinuierlicher Übergang von schallharter zu nachgiebiger Wand - ist es durchaus denkbar, daß die beiden Unbekannten ebenfalls sehr singulär gewählt werden müssen, damit die Rechnung physikalisch richtige Amplituden für die ausbreitungsfähigen Moden liefert.

Genauso denkbar ist, daß eine solche Wahl gar nicht möglich, das Modell von Koch und Möhring also schon zu grob für das betrachtete Phänomen ist.

In Anhang $\mathrm{C}$ werden Gleichungen vorgestellt, die $\eta(0)$ und $\eta(l)$ erfüllen müssen, damit Wechseldruck oder Auslenkung der Wirbelschicht an den Übergängen stetig oder zumindest endlich bleiben. Einige Kombinationen sind in Tabelle 5.1 zusammengestellt.

Der Eintrag KuTTA besagt dabei, daß sowohl die Auslenkung als auch die Ableitung der Auslenkung nach $x$ verschwinden - die Wirbelschicht die Hinterkante des stromauf gelegenen, schallharten Rohres also tangential verläßt.

Die widersprüchlich erscheinenden Einträge in der ersten und fünften Zeile, nach denen $\eta(l)=0$ trotzdem auf unendlich große Wirbelschicht-Auslenkungen bei $x=l$ führen kann, erklären sich so:

Berechnet man $\eta(l)$ aus der Fouriertransformierten, die die WIENER-HopfMethode liefert, so ist dieser Wert (wegen der Asymptotik der Transformierten) 
a priori unendlich, es sei denn, es ist die Gleichung (C.39) erfüllt. $\eta(l)=0$ läßt die Gleichung aber nicht automatisch erfüllt sein, so daß man trotzdem formal unendlich erhält, weil die Transformierte nicht schnell genug abfällt.

Welche Sprungbedingungen bieten sich an?

Naheliegend ist, zumindest $\eta(0)=0 \mathrm{zu}$ fordern, da man mindestens ein stetiges Ablösen der Wirbelschicht am Einlauf in das Meßobjekt erwarten würde. Die instationäre KUTTA-Bedingung ist dann automatisch erfüllt. Diese Eigenschaft liegt nicht vor, wenn die Instabilitätsmode akausal als stromauf laufende Welle in der Rechnung berücksichtigt wird, siehe Fälle A-C in [10]. Offenbar sorgt die instabile Mode dafür, daß die KUTTA-Bedingung am stromauf liegenden Sprung erfüllt wird, sofern die Wirbelschicht dort zumindest stetig anliegt.

Die plausibelste Wahl am Stromab-Ende der Auskleidung ist sicherlich Gleichung (C.39), wodurch Auslenkung und Druck bei $x=l$ endlich bleiben ${ }^{3}$. Ich werde für den Vergleich zwischen Rechteckgeometrie und den experimentellen Daten ausschließlich diesen Fall untersuchen und die anderen Sprungbedingungen nur im Kanal mit kreisförmigem Querschnitt.

\subsubsection{Ergebnisse für den Rechteckkanal}

In den Abbildungen 5.3, 5.4 sind die experimentellen Daten für eine typische Machzahl mit den Resultaten einer Streurechnung für den Rechteckkanal verglichen. Die Sprungbedingungen habe ich, wie bereits erwähnt, so gewählt, daß Druck und Auslenkung an den Sprungstellen endlich bleiben und am StromaufEnde die KuTTA-Bedingung erfüllt ist ${ }^{4}$.

Im nachgiebig ausgekleideten Rohr wurden vierzehn Moden-sechs stromauf, acht stromab - bis zu den fünften höheren Moden berücksichtigt. Die unterschiedlichen Zahlen ergeben sich aus den zusätzlichen Lösungen, die beide stromab laufen. Dabei ist es unerheblich zu wissen, welche der Moden diese zusätzlichen Lösungen sind. Wichtig ist lediglich, daß die instabile Mode bei den stromab laufenden erscheint.

Es sei darauf hingewiesen, daß die bereits in [7] veröffentlichten theoretischen Streufaktoren auf fehlerhaften Gleichungen beruhen, die hier berichtigt wurden.

\footnotetext{
${ }^{3}$ Diese Gleichung ist hinreichend, im mathematischen Sinne aber offenbar nicht notwendig, da distributions- und funktionentheoretische Regularisierungen denkbar sind, die für die eigentlich divergenten Integrale trotzdem endliche Werte liefern, [15].

${ }^{4}$ Im Druck von [10] befinden sich zwei Tippfehler: In der ersten Gleichung von (19d) muß es auf der rechten Seite $\overleftarrow{R}_{m, n}$ (statt $\overleftarrow{R}_{m, s}$ ) heißen. In Gleichung (38) ist im letzten Summanden ein Vorzeichen falsch, dort muß $\left(\gamma_{m, 2 \sigma}+K / M a\right)$ statt $\left(\gamma_{m, 2 \sigma}-K / M a\right)$ stehen.
} 

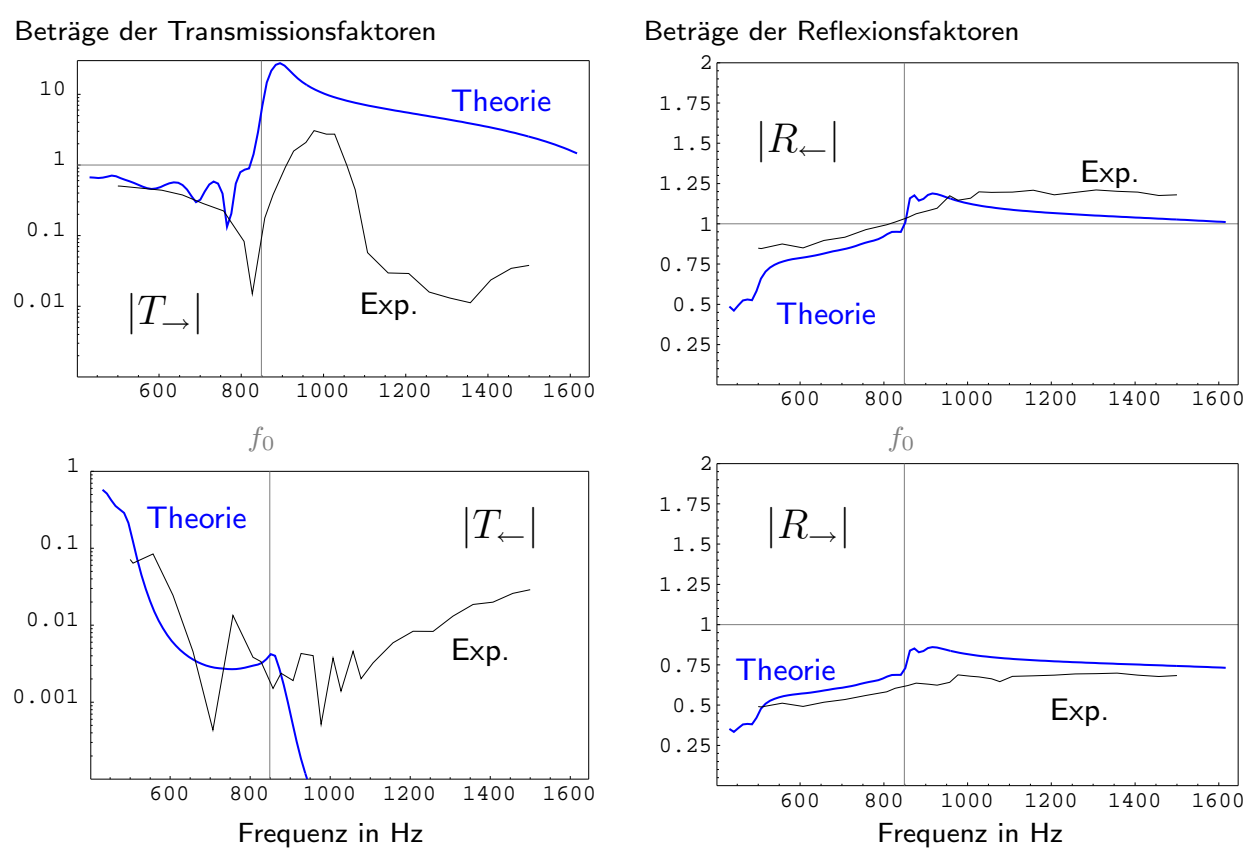

Abbildung 5.3: Streufaktoren für ein Meßobjekt der Länge $l_{0}=8,75 \mathrm{~cm}$, Kanalbreite $h_{0}=5 \mathrm{~cm}$ und MACH-Zahl 0,16, verglichen mit den experimentellen Daten im Rohr

Während die Reflexionsfaktoren trotz unterschiedlicher Geometrie bereits annähernd richtig wiedergegeben werden, stimmen die Beträge der Transmissionsfaktoren nur bis zur Resonanzfrequenz mit den experimentellen Daten überein. Die Phase von $T \rightarrow$ scheint einen gänzlich anderen Verlauf zu nehmen, obwohl es hier den Anschein hat, als gäbe es einen Vorzeichenkonflikt in der Definition. Da Brandes und Koch, MÖHRING allerdings dieselbem Phasenkonventionen benutzen, ist davon nicht auszugehen.

Schon in diesem Vergleich zeigt sich, daß die Anfachungsrate der instabilen Mode für dem Transmissionsfaktor zwar eine entscheidende Rolle spielt - auch $\left|T_{\rightarrow}\right|$ steigt, ebenso wie $-\operatorname{Im} k_{x}$, bei $f_{0}$ sprunghaft an - dieser aber trotzdem einen davon abweichenden Verlauf nehmen kann. So ist in Abbildung 3.11 zu sehen, daß die Anfachung für hohe Frequenzen in etwa konstant bleibt, obwohl der Transmissionsfaktor in diesem Bereich, wenn auch nicht so stark wie im Experiment beobachtet, absinkt. 


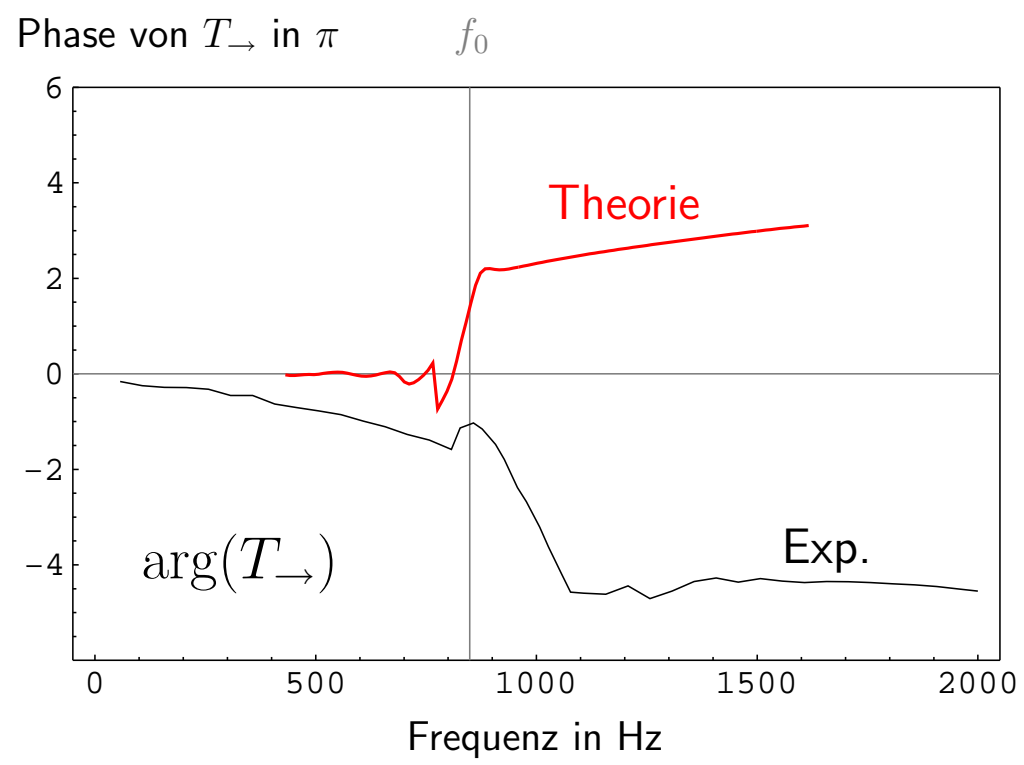

Abbildung 5.4: Phase des Transmissionsfaktors $T \rightarrow$ aus der vorherigen Abb.

\subsection{Rohrgeometrie}

Die Analyse des vorherigen Abschnittes läßt sich ohne weiters auf einen Kanal mit kreisförmigem Querschnitt übertragen. Die Formeln dazu habe ich in Anhang C zusammengestellt. Sie unterscheiden sich nur an wenigen Stellen von denen eines Rechteckkanals und lassen sich mit denselben Methoden wie in [10] gewinnen. Ich habe in der numerischen Rechnung wieder Moden bis zur fünften höheren Ordnung berücksichtigt.

\subsubsection{Ergebnisse für das Rohr}

Überraschenderweise stellt sich heraus, daß die marginal erscheinenden Änderungen und das recht ähnliche Modenspektrum zu völlig anderen Ergebnissen führen, wie in den Abbildungen 5.6 und 5.7 deutlich wird. Obwohl nur die Kanalgeometrie geändert wurde, wird der Transmissionsfaktor in Grundströmungsrichtung bei der dargestellten MACH-Zahl um ein bis zwei Ordnungen größer als beim Rechteckkanal. Er ist auch viel größer als im Experiment beobachtet.

Für $T_{\leftarrow}$ liefert die Rechnung mit Null verträgliche Werte. Die Reflexionsfaktoren stimmen der Größenordnung nach, es gibt aber bei $R_{\leftarrow}$ erhebliche Abweichungen. 

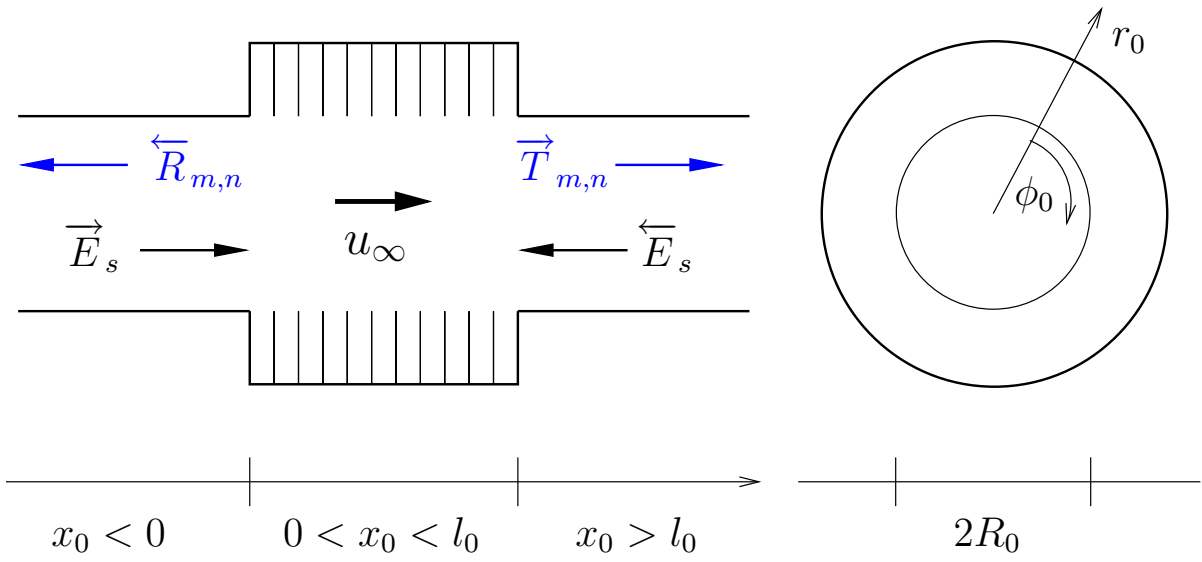

Abbildung 5.5: Ein nachgiebig ausgekeidetes Rohrsegment

Beträge der Transmissionsfaktoren
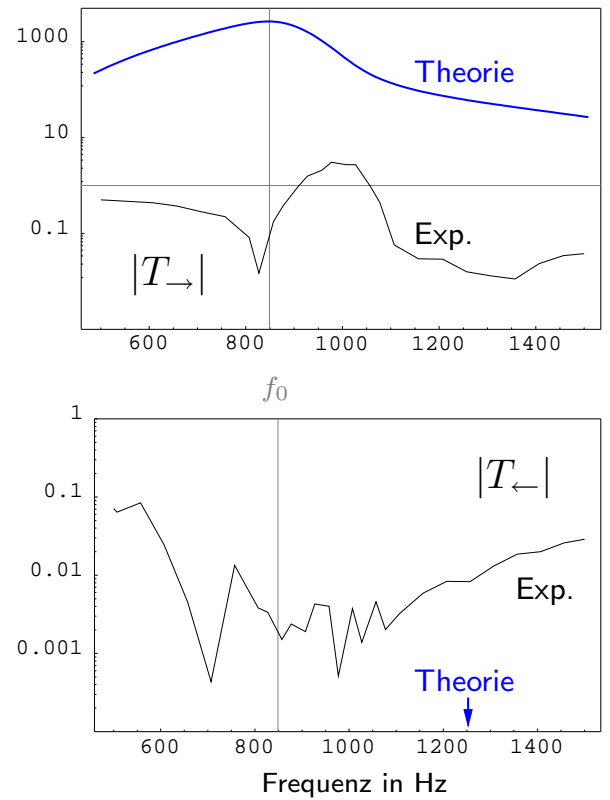

Beträge der Reflexionsfaktoren
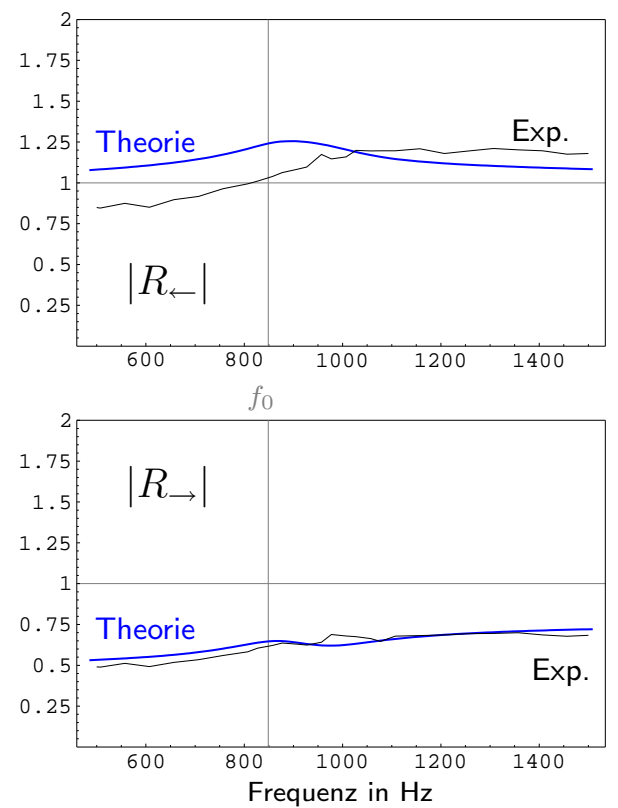

Abbildung 5.6: Streufaktoren für ein Rohrstück der Länge $l_{0}=8,75 \mathrm{~cm}$, Durchmesser $h_{0}=5 \mathrm{~cm}$, MACH-Zahl 0,16, verglichen mit den experimentellen Daten. 


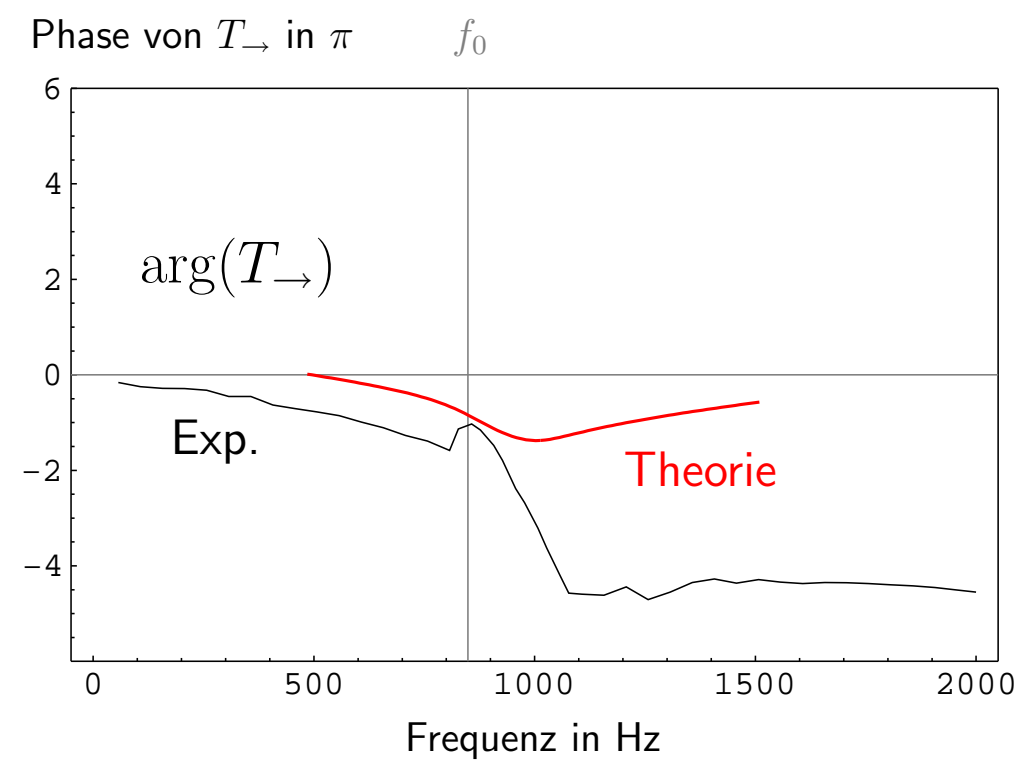

Abbildung 5.7: Phase des Transmissionsfaktors $T \rightarrow$ im Rohr.

$\arg \left(T_{\rightarrow}\right)$ hat jetzt das richtige Vorzeichen, allerdings ist die Phasendrehung nahe der Resonanz zu gering.

Es stellen sich zwei Fragen:

1. Woher kommen die großen Unterschiede bei unterschiedlicher Kanalgeometrie?

2. Gibt es Sprungbedingungen, für die die Übereinstimmung besser ist?

Um der ersten Frage nachzugehen, habe ich das Verhalten einzelner Terme im hier am meisten interessierenden Streufaktor $T_{\rightarrow}$ untersucht. Dabei hat sich herausgestellt, daß dieser große Werte annimmt, weil die Auslenkung am Stromab-Ende $\eta(l)$ großwird (siehe dazu Formel (C.48) im Anhang). Das ist auch physikalisch plausibel: Wenn die Wirbelschicht dort stark auslenkt wird, sollte die Schallabstrahlung größer werden.

Eine Konsequenz daraus ist, daß bei den Sprungbedingungen $\eta(0)=\eta(l)=0$ die Schallverstärkung ausbleibt, wie man in Abbildung 5.8 sieht.

Der Faktor, der $\eta(l)$ groß werden läßt, ist im Falle der bisher gewählten Bedingung (C.39) 

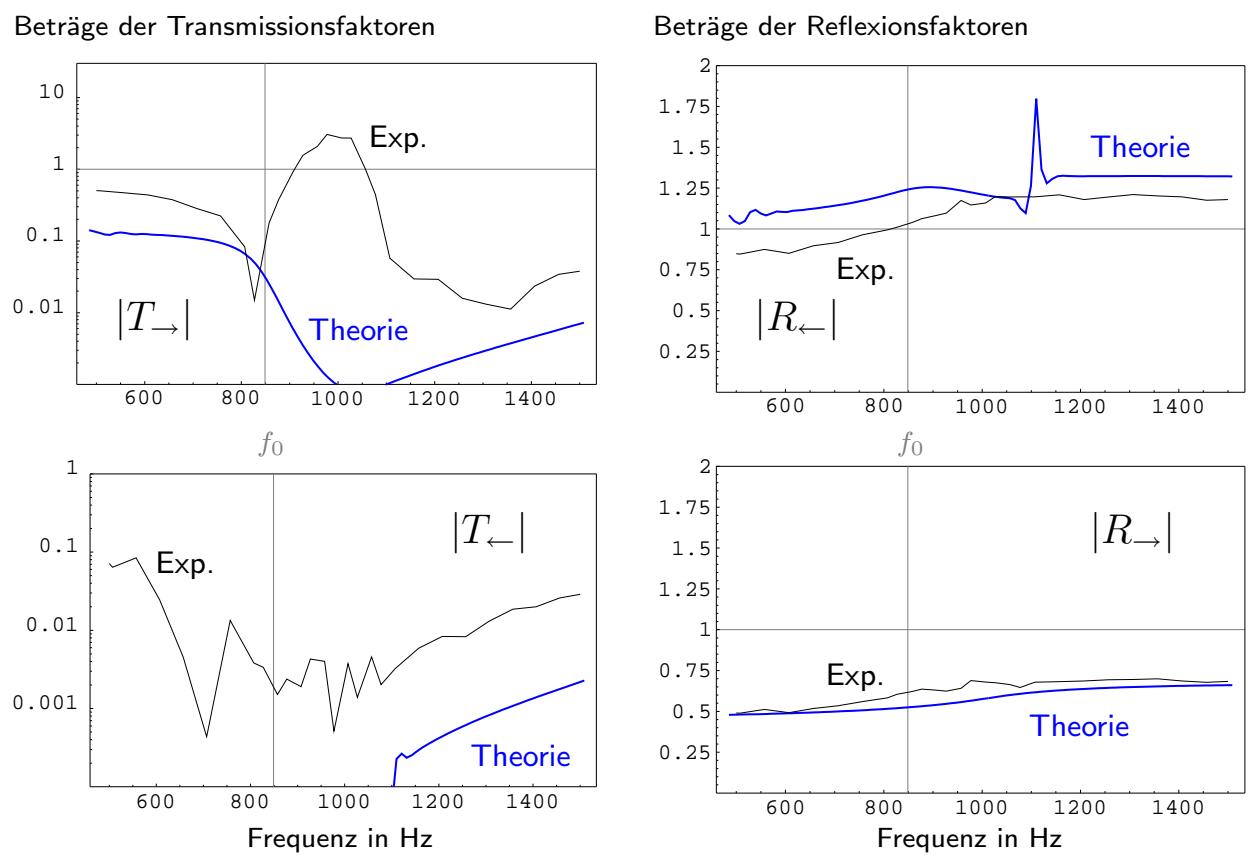

Abbildung 5.8: Streufaktoren für ein Rohrstück, aber mit $\eta(0)=\eta(l)=0$, MACHZahl 0,16. Die Oszillation bei $1100 \mathrm{~Hz}$ ist offenbar ein Artefakt, das kleiner wird, wenn man die Zahl der Moden erhöht - deswegen hier Berücksichtigung von achtzehn statt vierzehn Moden.

$$
\exp \left(i \lambda_{0,-2}^{+} l\right)
$$

der in Gleichung (C.39) über $Q_{n}$ eingeht, siehe dazu Definition (C.31). Auch dieses Verhalten ist physikalisch einsichtig, den dieser Ausdruck ist nichts anderes als der Anfachungsfaktor der instabilen Mode.

Den Unterschied zwischen Rechteckkanal und Rohr macht einer der verbleibenden Faktoren in $\eta(l)$ aus $^{5}$ : Dieser nimmt im ersten Fall einfach sehr kleine Werte an, ohne daß ich dieses Verhalten auf einen einzelnen Term zurückführen kann, da der Faktor Lösung einer großen Matrizengleichung ist (deren Umfang mit der Zahl der berücksichtigten Moden wächst).

Diese Erscheinung kann anschaulich etwa so gedeutet werden: Zwar sind die räumlichen Anfachungsfaktoren der instabilen Mode in beiden Geometrien sehr groß

\footnotetext{
${ }^{5}$ Es ist $u_{\oplus}^{*}\left(\lambda_{0,-2}^{+}\right)$.
} 
(tausend und mehr), im Rechteckkanal wird die Instabilität aber für höhere Frequenzen und die hier gewählten Sprungbedingungen weit weniger angeregt als im Rohr.

Falls diese Unterschiede in der Anregung der Instabilität der Wirklichkeit entsprechen, könnten sich Kanäle mit unterschiedlichen Querschnitts-Geometrien sehr verschieden verhalten, selbst wenn sich ihre Modenspektren ähneln.

Die riesigen Änderungen, die kleine Abweichungen der Moden (genauer: ihrer Wellenzahlen) mit sich bringen können, erklären sich aus den an vielen Stellen vorkommenden Quotienten

$$
\frac{1}{k^{i}-k^{j}}
$$

Selbst wenn in zwei Modellen 1, 2 die Wellenzahlen sehr ähnlich verlaufen,

$$
k_{1}^{i} / k_{2}^{i} \approx 1
$$

können sich trotzdem die Inversen von Differenzen zweier Wellenzahlen von Modell zu Modell um Größenordnungen unterscheiden - mit entsprechend weitreichenden Auswirkungen für die numerischen Resultate.

Zur Klärung der zweiten Frage habe ich auch andere Kombinationen von Sprungbedingungen - siehe Tabelle 5.1-getestet, auch wenn sie physikalisch nicht unbedingt naheliegend sind. Dabei hat sich herausgestellt, daß diese anderen Kombinationen nahezu identische Resultate wie die bereits beschriebenen liefern:

1. $\eta(l)=0$, Gl. (C.39) oder $\eta(l)=0$, Gl. (C.36): wie $\eta(0)=\eta(l)=0$

2. $\eta(0)=0$, Gl. (C.36) oder Gl. (C.39), Gl. (C.36): wie $\eta(0)=0$, Gl. (C.39)

Wird die Auslenkung der Wirbelschicht am Stromab-Ende des Meßobjektes auf Null gesetzt, gibt es keine Schallverstärkung stromab, unabhängig davon, welche Bedingung man am stromauf gelegenen Ende fordert. Umgekehrt liefern Gleichungen, die $\eta(l) \neq 0$ zulassen, stets Schallverstärkung - um Größenordnungen über der beobachteten. Obwohl es sechs mögliche Kombinationen von Forderungen an $\eta(0)$ und $\eta(l)$ gibt, scheint es in der Formulierung der Sprungbedingungen noch gewisse Redundanzen zu geben.

Von Interesse ist auch die Abhängigkeit der Streufaktoren von der Strömungsgeschwindigkeit. BRANDES hatte einen sich verbreiternden und höher werdenden 


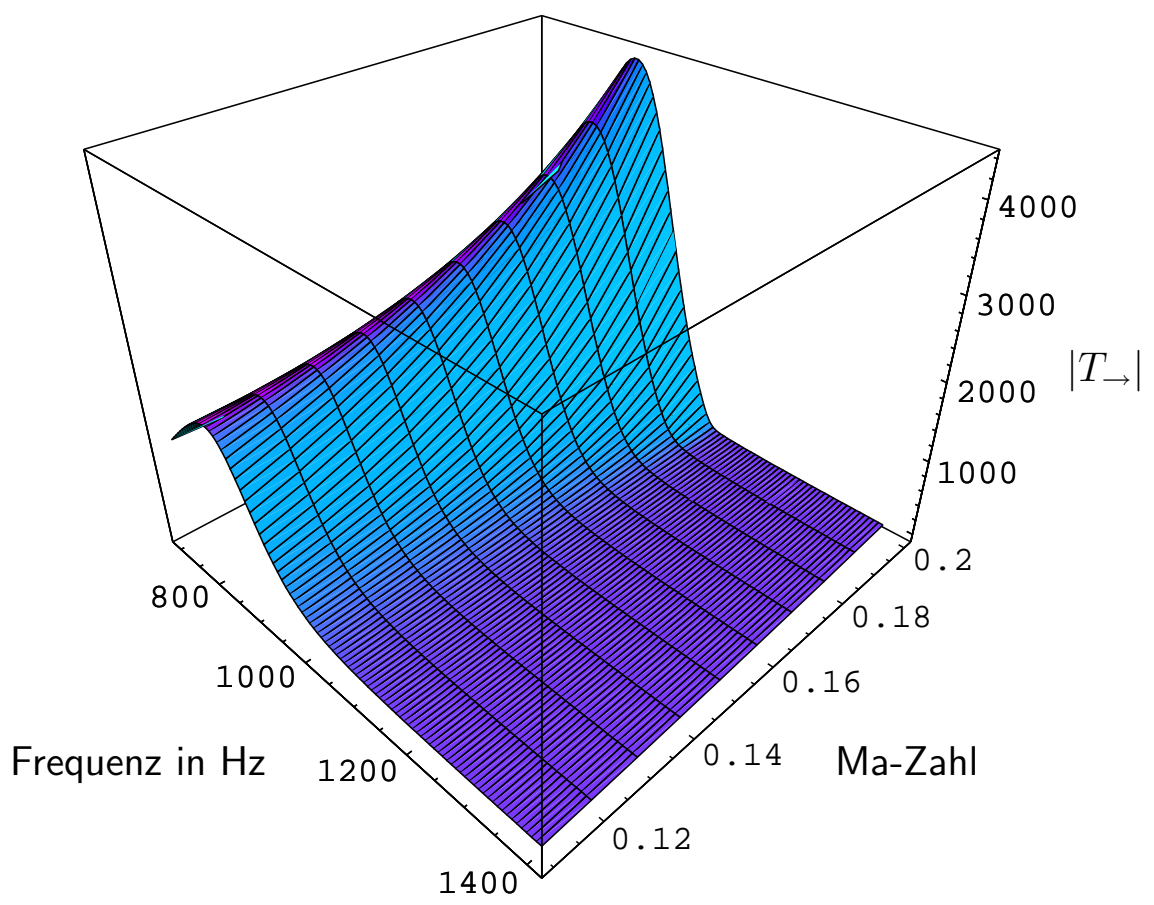

Abbildung 5.9: Betrag des Transmissionsfaktors $T \rightarrow$ als Funktion der Frequenz und der Machzahl. Sprungbedingungen $\eta(0)=0 ; \eta(l)$ sind so gewählt, daß Druck und Auslenkung bei $x=l$ endlich bleiben.

Peak bei zunehmender MACH-Zahl beobachtet. Dem stand die Erkenntnis des Kapitels 3 entgegen, nach der die Anfachung der Instabilitätsmode für wachsendes $u$ abnimmt.

Diese anscheinend gegensätzlichen Beobachtungen lassen sich klären, wenn man $T \rightarrow$ als Funktion von Frequenz und MACH-Zahl darstellt, was in Abbildung 5.9 geschehen ist. Dort steigt der Betrag des Transmissionsfaktors, obwohl die Instabilitätsmode weniger stark angefacht ist. Der Imaginärteil der Mode allein entscheidet also nicht, wie die Verstärkung verläuft.

\subsection{Zusammenfassung}

Ich habe in diesem Kapitel untersucht, ob eine Berechnung der Streufaktoren mit den Methoden aus [10] zu sinnvollen Ergebnissen führt. 
Das scheint nicht der Fall zu sein.

Allerdings ist diese Analyse trotzdem aufschlußreich, weil die Wirkung der Sprungbedingungen weiter aufgeklärt wird.

Es zeigt sich, daß am Stromab-Ende eine endliche Auslenkung der Wirbelschicht gefordert werden muß, da es andernfalls zu keiner Schallverstärkung kommt. $T_{\rightarrow}$ ist jedoch viel zu groß und zeigt nicht den charakteristischen Peak oberhalb der Resonanzfrequenz.

Von den Reflexionsfaktoren stimmt $R_{\rightarrow}$ recht gut, $R_{\leftarrow}$ zumindest in der Größenordnung mit dem Experiment überein. $T_{\leftarrow}$ wird viel zu klein vorhergesagt.

Obwohl sich die räumliche Anfachung der instabilen Mode mit zunehmender Strömungsgeschwindigkeit verringert, nimmt die vorhergesagte Verstärkung in Stromab-Richtung trotzdem zu. Das Anfachungsverhalten der Mode allein ist also noch nicht auschlaggebend.

Das Ziel, das akustische Verhalten der Kanalsegmente vorhersagen zu können, kann nur erreicht werden, wenn der Verlauf der Moden sehr genau bekannt istdie Streurechnung reagiert sehr empfindlich darauf, wenn Lösungen ähnliche Wellenzahlen aufweisen - und geeignete Forderungen an den Diskontinuitäten $x=0$, $x=l$ gestellt werden. Es ist nicht gesagt, daß die beste Wahl sich unter den hier vorgestellten Kombinationen von Sprungbedingungen befindet.

Die Rechnung reagiert ebenfalls empfindlich auf die Zahl der berücksichtigten Moden. Da keine Chance bestand, mit diesem Modell das experimentelle Ergebnis zu reproduzieren, habe ich es hier aus Zeitgründen bei maximal achtzehn Moden belassen, was inklusive der beiden Auslenkungen $\eta(0), \eta(l)$ auf ein Gleichungssystem mit zwanzig Unbekannten führt.

Es mag enttäuschen, daß der Transmissionsfaktor um Größenordnungen über dem beobachteten liegt. Das ist aber nicht anders zu erwarten, da sich Abweichungen in den Wellenzahlen exponentiell auf den Transmissionsfaktor auswirken, ganz abzusehen von nichtlinearen Sättigungs- oder sogar destruktiven Effekten, die hier ja überhaupt nicht berücksichtigt sind. Im Idealfall hätte man erwarten können, vielleicht die ungefähre Lage und Breite des Verstärkungsbereiches zu reproduzieren. 


\section{Kapitel 6}

\section{Ergebnisse und Ausblick}

Ziel dieser Arbeit war die Aufklärung der physikalischen Natur der Instabilität Typ I und eine bestmögliche mathematische Modellierung.

Es hat sich herausgestellt, daß dieses Ziel nur mit einem erheblichen Aufwand $\mathrm{zu}$ erreichen ist. Ich halte es für unwahrscheinlich, daß die wesentlichen Eigenschaften des Verstärkungsphänomens (etwa Lage, Breite und Höhe des Peaks in Abhängigkeit von der Machzahl) im Rahmen eines einfachen Modells reproduziert werden können.

Die Existenz einer konvektiven hydrodynamischen Instabilität in allen in Frage kommenden Modelle kann als gesichert gelten. Es darf davon ausgegangen werden, daß sie auch natürlicherweise existiert und die Ursache für das Verstärkungsphänomen Typ I ist.

Den ersten Schwerpunkt dieser Arbeit bildete die Analyse der Instabilität in unterschiedlich stark vereinfachten, linearen Modellen für das Innere des ausgekleideten Kanalstücks allein. Dabei wurden die folgenden Beobachtungen gemacht:

Alle Modelle besitzen einen Instabilität, solange nur eine Grundströmung und eine resonanzartig nachgiebige Wand vorhanden ist. Zerlegt man die Lösungsvielfalt in Rohrmoden, so tauchen zwei zusätzliche, stromab laufende Moden auf, von denen eine in Ausbreitungsrichtung angefacht ist. Für die Festlegung der Ausbreitungsrichtung habe ich mich auf das distributionstheoretische Kriterium von Jones und Morgan gestützt. Nahe der Resonanzfrequenz der Wand nimmt in allen Modellen die Anfachung sprunghaft zu, während der Realteil der Ausbreitungswellenzahl stark absinkt. Unterhalb der Resonanzfrequenz ist dieser viel größer, die Anfachung hingegen viel kleiner, sofern das Modell keine Zähigkeit berücksichtigt, die gedämpftes Verhalten bei niedrigen Frequenzen bewirkt.

Da die Instabilität einer nachgiebigen Wand bedarf, war es naheliegend, davon 
auszugehen, daß sie in unmittelbarer Nähe der Wand lokalisiert ist. Diese Annahme hat sich als nur teilweise richtig herausgestellt: Die Eindringtiefe der instabilen Mode ist so groß, daß diese den größten Teil des Kanalquerschnittes ausfüllt.

In keinem Modell ist zu erkennen, daß die räumliche Anfachung bei hohen Frequenzen zusammenbricht. Mit steigender Strömungsgeschwindigkeit nimmt diese außerdem ab, obwohl die Verstärkung im Experiment mit der MACH-Zahl zunimmt.

Im zweiten Teil der Arbeit habe ich daher untersucht, welche Streufaktoren aus den Modenspektren resultieren, und dafür die Formeln von KocH und MöHrInG benutzt. Neben dem dort betrachteten Kanal mit rechteckigem Querschnitt habe ich das akustische Verhalten eines Rohrsegmentes mit den Abmessungen des Experiments untersucht.

Es hat sich gezeigt, daß Schallverstärkung (wenn auch viel größere als im Experiment beobachtet) nur eintritt, wenn eine endliche Auslenkung der Wirbelschicht am Stromab-Ende des Meßobjektes zugelassen wird. Die Verstärkung nimmt mit steigender MACH-Zahl zu, obwohl die Anfachungskonstante der Instabilitätsmode das entgegengesetzte Verhalten zeigt:

Zwar trägt die räumliche Anfachung der instabilen Mode über die Länge des Meßobjektes wesentlich zur Größe des Transmissionsfaktors bei, sie ist aber nicht allein entscheidend, wie sich an den sehr unterschiedlichen Verläufen für Flachkanal und Rohr und in der MACH-Zahl-Abhängigkeit zeigt.

Die Forderungen, die man an den Kontaktstellen zwischen den Meßrohren und dem kassettierten Kanalstück stellt, sind ebenfalls wesentlich. Als nächstliegende, wenn auch nicht einzig denkbare Wahl habe ich diskutiert:

- Auslenkung der Wirbelschicht ist stetig am Einlauf, $\eta(0)=0$. Die Auslenkung erfüllt dort dann auch automatisch die instationäre KUTTABedingung: $\partial_{x} \eta(0)=0$.

- Wechseldruck und Wirbelschichtauslenkung bleiben am Auslauf $x=l$ endlich, Gleichung (C.39).

Soll die Schallverstärkung, etwa für strömungsakustische Anwendungen, erhöht werden, genügt es daher nicht, nur nach Auslegungen zu suchen, in denen die instabile Mode räumlich stärker angefacht ist. Ohne Kenntnis der Anregungsmechanismen sind Vorhersagen über das Verstärkungsverhalten spekulativ.

Der starke Einfluß der Form des Kanalquerschnittes ist überraschend. Der Transmissionsfaktor wird sogar um Größenordnungen verschieden vorhergesagt. Zwar 
ist es spekulativ, von den hier vorgestellten, offensichtlich unzulänglichen Berechnungen auf die physikalische Wirklichkeit zu schließen, aber neuere Messungen [9] an einem Meßobjekt mit rechteckigem Querschnitt und gegenüberliegenden Resonatoren, vergleichbar der Anordnung in [10], zeigen das Verstärkungsphänomen überhaupt nicht, was diese These stützt.

Für ein besseres Verständnis des Experimentes ist es wünschenswert, die Felder im ausgekleideten Kanalstück zu kennen. Hier lagen bisher nur die Drucksondenmessungen aus [3] vor. Ohne diese Kenntnis hat man keine Anhaltspunkte, ob die Modellierung des Kanalinneren, insbesondere der instabile Mode, der physikalischen Realität nahekommen. Von den Streufaktoren auf die Güte dieser Modellierung zurückzuschließen ist wenig erfolgversprechend, weil neben die Unkenntnis der Sprungbedingungen noch nichtlineare Effekte treten, die die Amplitude der Instabilität begrenzen.

In dieser Arbeit nicht vorgestellt wurden Modelle, in denen die endliche Kammerbreite der Kassettierung aufgelöst wird. Wie bereits erwähnt hatten erste Rechnungen - allerdings nur für eine einzelne ebene Wand - keine Hinweise darauf ergeben, daß damit die Unzulänglichkeiten der Modelle mit lokal nachgiebigen Wänden gelöst würden. Es ist allerdings nicht auszuschließen, daß die Verhältnisse in einem periodisch ausgekleideten Rohrstück anders sind. Die Berechnung der Streufaktoren einer solchen Konfiguration wäre daher wünschenswert, wenn auch bei weitem aufwendiger als für den Fall lokal nachgiebiger Wände.

Andere Erweiterungen sind ebenfalls denkbar: So hat sich herausgestellt, daß die Berücksichtigung von Verlusttermen zumindest für niedrige Frequenzen die Instabilität zusammenbrechen läßt. Es wäre daher naheliegend, eine Berechnung der akustischen Streufaktoren unter Berücksichtigung etwa einer empirischen turbulenten Viskosität zu versuchen, um zu sehen, ob ein solches Modell besser ist. 


\section{Anhang A}

\section{Zur Ausbreitungsrichtung nach Briggs, Jones und MORGAN}

In [4] gibt BRIGGS ein einfaches Kriterium an, um die Ausbreitungsrichtung von Lösungen mit komplexer Wellenzahl zu ermitteln. Es lautet sinngemäß:

In einem Wellenproblem gebe es die Moden $i \in I$. Sei

$$
\sigma=\sup _{k \in \mathbb{R}} \operatorname{Im} \omega^{(i)}(k)
$$

die kleinste obere Schranke der Anfachungsrate $\operatorname{Im} \omega^{(i)}$.

Variiert man die Kreisfrequenz von $\omega$ bis $\omega+i \sigma$ und ändert dabei $\operatorname{Im} k_{x}^{(i)}(\omega)$ sein Vorzeichen,

$$
\operatorname{sign}\left(\operatorname{Im} k_{x}^{(i)}(\omega)\right) \neq \operatorname{sign}\left(\operatorname{Im} k_{x}^{(i)}(\omega+i \sigma)\right)
$$

so ist die Mode (i) instabil und breitet sich in Anfachungsrichtung aus. Andernfalls ist sie gedämpft.

Die Herleitung dieses Kriteriums stützt sich auf Funktionentheorie und LAPLACETransformation eines realistischen Problems, in dem eine Quelle nur in einem kompakten Raumgebiet $x \in(-\epsilon, \epsilon)$ existiert und zum Zeitpunkt $t=0$ eingeschaltet wird. Die Forderung, daß die Lösung für alle Zeiten $t<0$ verschwinden muß, übersetzt sich nach der Transformation in Analytizitätsforderungen an die Transformierte der Lösungsfunktion. Das erzwingt eine bestimmte Verteilung der Moden - in Gestalt der Pole $k_{x}^{(i)}(\omega)$-auf die Sektoren $x<0$ und $x>0$. 

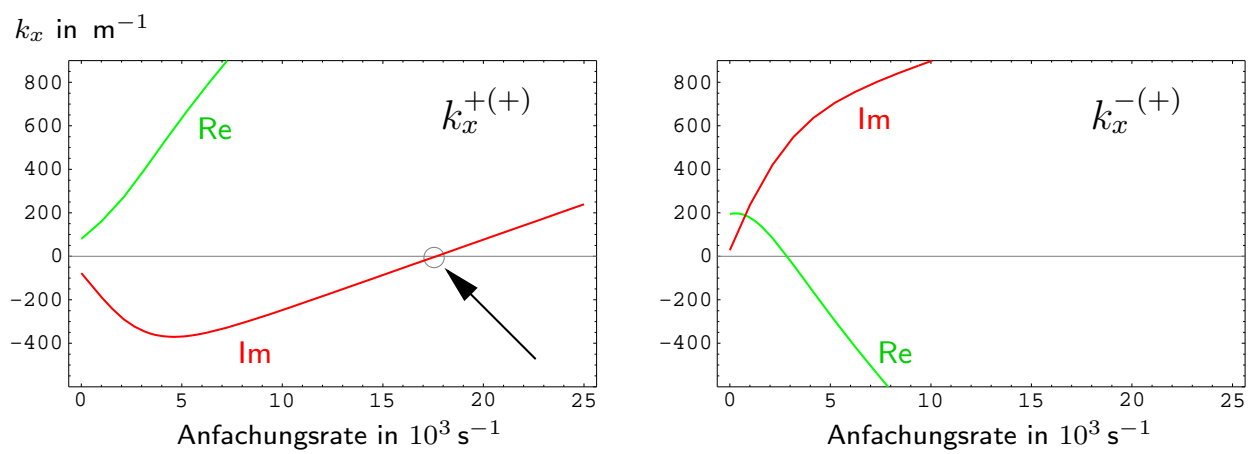

Abbildung A.1: Wellenzahlen der hydrodynamischen Moden für Re $f=900 \mathrm{~Hz}$ fest in Abhängigkeit vom Imaginärteil der Kreisfrequenz $\omega$. Der Imaginärteil von $k_{x}^{+(+)}$ wechselt das Vorzeichen, $k_{x}^{-(+)}$dagegen bleibt stromab räumlich gedämpft.

Im Falle der in 3.1 vorgestellten Lösungen $k_{x}^{ \pm( \pm)}$ist allerdings $\sigma$ unendlich, weswegen ich dieses Kriterium nicht verwenden kann. Um die Idee zu illustrieren, zeige ich trotzdem das Verhalten der hydrodynamischen Lösungen für eine Frequenz im Verstärkungsbereich.

In Abb. A.1 sieht man den erwähnten Wechsel der Dämpfungsrichtung für die Mode $k_{x}^{+(+)}$. Wie bereits erwähnt, ist das kein strenges Argument, weil die Voraussetzungen nicht erfüllt sind. Es kommt sogar eine offensichtlich falsche Ausbreitungsrichtung, nämlich für die Mode $k_{x}^{-(-)}$, heraus.

In [8] präsentieren Jones und MORGAn eine allgemeinere Analyse, auf die ich mich stütze werde. Sie kann wie folgt zusammengefaßt werden:

Eine Lösung ist genau dann kausal, wenn für festes $x$ die FOURIERTransformierte $\phi(\omega, x)$ in der oberen $\omega$-Halbebene regulär ist und reelle Zahlen $b, d>0$ existieren, so daß im Grenzfall $|\omega| \rightarrow \infty, \operatorname{Im} \omega \geq \epsilon>0$

$$
e^{-(b+i d) \omega} \phi(\omega, x)=O\left(|\omega|^{s}\right)
$$

für einen endlichen Exponenten $s$ ist. $\phi(t, x)$ verschwindet dann für $t<d$.

Die Autoren von [8] benutzen distributionstheoretische Methoden, um dieses Kriterium zu beweisen. Es sieht zunächst dem BRIGGsschen Argument sehr unähnlich, aber für den Fall, daß für große $\omega$ die Dispersionsbeziehung einer Mode 
asymptotisch linear wird,

$$
k(\omega) \sim(\alpha+i \beta) \omega
$$

kann man die linke Seite vom A.1 asymptotisch bestimmen,

$$
\begin{aligned}
e^{-(b+i d) \omega} \phi(\omega, x) & =e^{-(b+i d) \omega+i(\alpha+i \beta) \omega x} \\
& =e^{(-\beta x-b) \operatorname{Re} \omega+(d-\alpha x) \operatorname{Im} \omega+i \ldots} \\
& \stackrel{!}{=} O\left(|\omega|^{s}\right)
\end{aligned}
$$

und untersuchen, ob man die Konstanten $b, d$ geeignet wählen kann. Der Grenzfall $\operatorname{Im} \omega=$ const., $\operatorname{Re} \omega \rightarrow+\infty$ erzwingt die Wahl

$$
b=-\beta x
$$

Der Grenzfall $\operatorname{Im} \omega \rightarrow+\infty$ führt zu der Forderung

$$
d<\alpha x
$$

Weil $d$ nach Voraussetzung auch noch positiv sein soll, ergibt sich als Kriterium für die Kausalität dieser Mode

$$
0<d<\alpha x
$$

Das geht nur, wenn wenigstens $0<\alpha x$ ist, oder wieder allgemeiner ausgedrückt (siehe Definition von $\alpha$ in (A.2)),

$$
0<\operatorname{Im} k(\omega)_{\omega \rightarrow+i \infty} \cdot x
$$

Kurzgefaßt: Wellenzahlen mit der Asymptotik (A.2), deren Imaginärteil für $\omega \rightarrow i \infty$ positiv wird, breiten sich nach rechts aus (existieren für $x>0$ ), wird er negativ, laufen sie nach links $(x<0)$. Der Unterschied zum Argument von BRIGGS besteht darin, daß der Imaginärteil der Wellenzahl für rein imaginäre Frequenzen und nicht etwa komplexe untersucht wird.

\section{Die akustische Mode $k_{x}^{-(-)}$}

In Abschnitt 3.1.5 habe ich behauptet, daß die Ausbreitungsrichtung mit Hilfe der kompressiblen Lösungen ermittelt werden muß. 

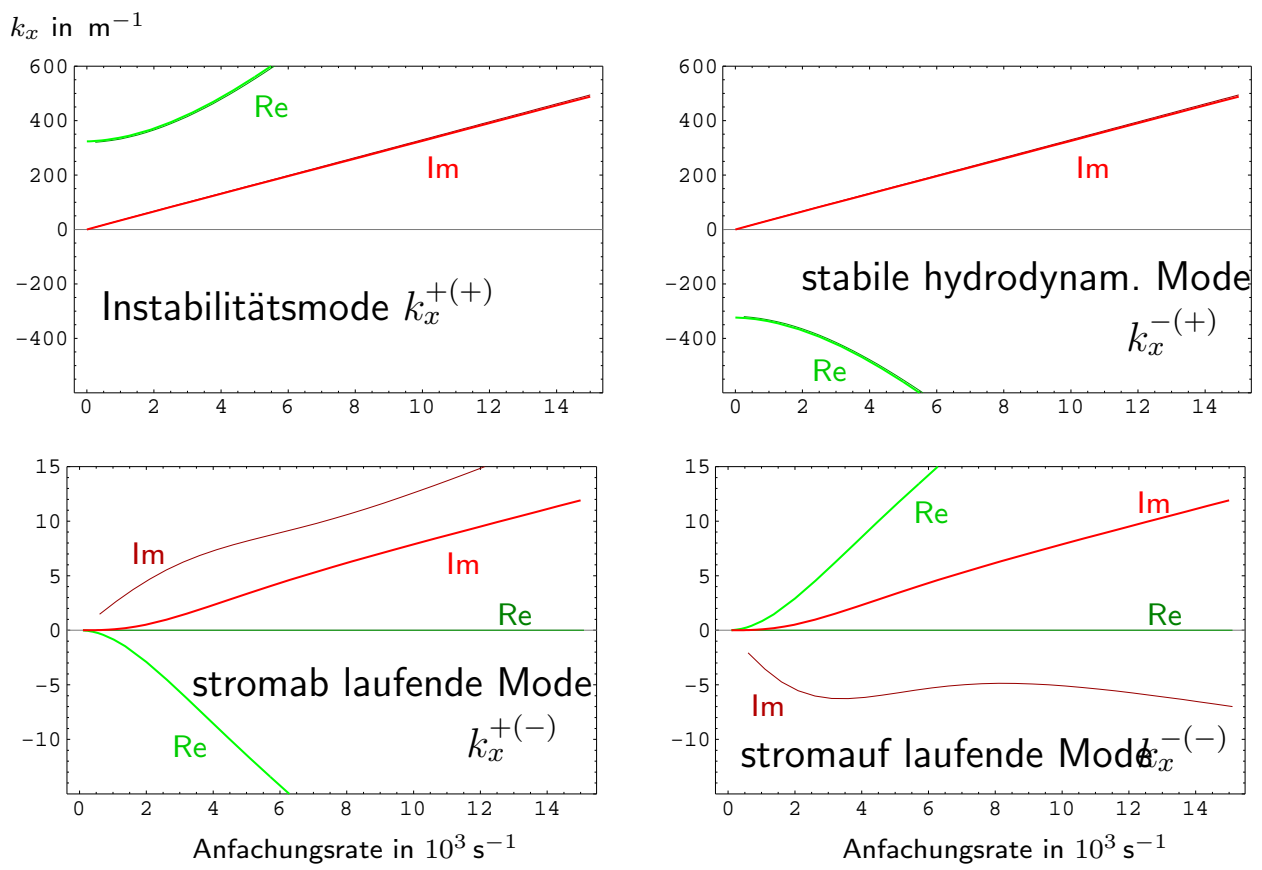

Abbildung A.2: Wellenzahlen der vier Lösungen in Abhängigkeit von der Anfachungsrate $\operatorname{Im} \omega$. dicke Linien: inkompressibel, dünne Linien: kompressibel

Wendet man den Formalismus von Jones, Morgan auf die inkompressibel gerechnete Mode $k_{x}^{-(-)}$an, so erhält man asymptotisch überraschenderweise Wellenzahlen mit positivem Imaginärteil-siehe Abb. A.2-was einer Ausbreitung in Grundströmungsrichtung gleichkäme.

Das ist zunächst beunruhigend, weil es die Gültigkeit dieser Analysen in Frage zu stellen scheint. Mir ist nicht klar, an welcher Stelle der entscheidende Unterschied, nämlich die endliche Schallgeschwindigkeit, in die mathematische Analyse eingeht, allerdings untersuchen auch die genannten Autoren ein Modell mit endlicher Kompressibilität. Möglicherweise ändert der Grenzfall $c \rightarrow \infty$ die funktionentheoretischen Eigenschaften der komplexen Funktion $k_{x}(\omega)$ derart, daß die in [8] genannten Sätze oder die asymptotische Analyse nicht mehr durchführbar sind. Ich bin dieser Frage nicht weiter nachgegangen.

Physikalisch ist ein derartiges Problem durchaus vorstellbar: Da im inkompressiblen Grenzfall die Schallgeschwindigkeit unendlich wird, können sich in dem entsprechenden Modell Wirkungen instantan ausbreiten, da es nun keine endli- 
che Ausbreitungsgeschwindigkeit mehr gibt. Eine Argumentation, der Kausalität zugrundeliegt, wäre dann möglicherweise hinfällig. 


\section{Anhang B}

\section{Die WiEnER-Hopf-Methode: Ein einfaches Beispiel}

Ich werde in diesem Anhang das Prinzip der WIENER-Hopf-Methode an einem Beispiel erläutern, nämlich der Streuung von Wellen an einer Kontaktfläche zwischen Medien mit unterschiedlich großen Schallgeschwindigkeiten (aber gleicher Dichte). Das ist kein besonders typischer Fall, da die Methode eigentlich darauf abzielt, Diskontinuitäten in den Randbedingungen zu behandeln, aber auch in diesem eindimensionalen Beispiel werden alle Schritte der Methode klar. Ich benutze wie in Kapitel 5 die Konvention $\exp (i \omega t+i k x)$.

Gesucht sind die Streufaktoren $R$ und $T$ in Abhängigkeit von der Frequenz einer eingestrahlten Welle.

Dieses Problem ist einfach und mit elementaren Methoden sofort lösbar.

\section{B.1 Lösungen mit elementaren Mitteln}

Rechtslaufende Wellen der Kreisfrequenz $\omega$ breiten sich in den Teilgebieten mit den Wellenzahlen

$$
\begin{aligned}
& k_{-}=-\frac{\omega}{c_{-}}, \quad x<0 \\
& k_{+}=-\frac{\omega}{c_{+}}, \quad x>0
\end{aligned}
$$

aus. Ich setze links eine Linearkombination aus einfallender Welle und reflektierter Welle an, während sich rechts eine Welle mit Transmissionsfaktor $T_{\rightarrow}$ ausbreitet 


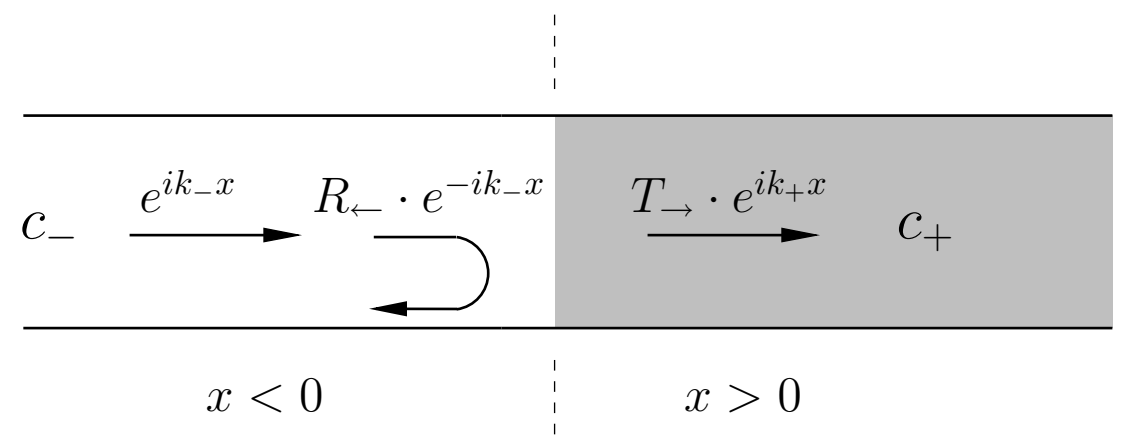

Abbildung B.1: Streuung am Übergang in ein anderes Medium. $c_{ \pm}$ist die Phasengeschwindigkeit in den Gebieten $x>0$ bzw. $x<0$.

(siehe Abb. B.1).

Welche Bedingungen man an der Kontaktfläche $x=0$ fordert, hängt von der Physik des Problems ab; im Falle des Wechseldrucks ist es die Stetigkeit der Wechselgröße, woraus

$$
1+R_{\leftarrow}=T_{\rightarrow}
$$

folgt, sowie die Stetigkeit der ersten Ableitung:

$$
k_{-}-k_{-} R_{\leftarrow}=k_{+} T_{\rightarrow}
$$

Löst man diese zwei Gleichungen nach den Streufaktoren auf, so erhält man das Ergebnis

$$
\begin{aligned}
T_{\rightarrow} & =\frac{2 c_{+}}{c_{+}+c_{-}} \\
R_{\leftarrow} & =\frac{c_{+}-c_{-}}{c_{+}+c_{-}}
\end{aligned}
$$




\section{B.2 Demonstration der WienER-Hopf-Methode}

Zur Bestimmung der Streufaktoren setze ich das Wechselfeld als eine im Gebiet $x<0$ einfallende Welle plus einem noch unbekannten Streufeld $\Phi$ an:

$$
\Phi_{\mathrm{ges}}=\left\{\begin{array}{cc}
e^{i k_{-} x}+\Phi(x) & , x<0 \\
\Phi(x) & , x>0
\end{array}\right.
$$

Damit die (auslaufenden) Streuwellen in $\Phi$ fouriertransformierbar bleiben, baue ich eine kleine Dämpfung ein, wodurch $k_{ \pm}$in $k_{ \pm}+i \epsilon$ übergeht.

Ich führe nun die halbseitigen FOURIER-Transformierten ein,

$$
\begin{aligned}
\Phi_{\oplus}(k) & =\int_{-\infty}^{0} d x \Phi(x) e^{-i k x} \\
\Phi_{\ominus}(k) & =\int_{0}^{+\infty} d x \Phi(x) e^{-i k x} \\
\Phi(k) & =\Phi_{\ominus}(k)+\Phi_{\oplus}(k)
\end{aligned}
$$

Jetzt suche ich die WIENER-HopF-Gleichung, welche die halbseitigen Transformierten und die Sprungbedingungen an der Diskontinuität miteinander verknüpft. Da die Schallgeschwindigkeit und damit die Differentialgleichung selbst die Diskontinuität beinhaltet, transformiere ich diese ebenfalls ${ }^{1}$. Die Wellengleichungen

$$
\begin{aligned}
& \left(k_{-}^{2}+\partial_{x}^{2}\right) \Phi(x)=0, \quad x<0 \\
& \left(k_{+}^{2}+\partial_{x}^{2}\right) \Phi(x)=0, \quad x>0
\end{aligned}
$$

werden nach FouRIER-Transformation auf den jeweiligen Halbachsen und partieller Integration $\mathrm{zu}$

\footnotetext{
${ }^{1}$ Liegt ein Sprung in den Randbedingungen vor, entsteht die WIENER-HopF-Gleichung in analoger Weise aus der Randbedingung.
} 


$$
\begin{aligned}
x<0: 0 & =\int_{-\infty}^{0} d x e^{-i k x}\left(k_{-}^{2}+\partial_{x}^{2}\right) \Phi(x) \\
& =\left(k_{-}^{2}-k^{2}\right) \Phi_{\oplus}+i k \Phi\left(0^{-}\right)+\partial_{x} \Phi\left(0^{-}\right) \\
x>0: 0 & =\int_{0}^{+\infty} d x e^{-i k x}\left(k_{+}^{2}+\partial_{x}^{2}\right) \Phi(x) \\
& =\left(k_{+}^{2}-k^{2}\right) \Phi_{\ominus}-i k \Phi\left(0^{+}\right)-\partial_{x} \Phi\left(0^{+}\right)
\end{aligned}
$$

Die FouRIER-Transformationen sind wegen der kleinen Dämpfung, welche das Streufeld asymptotisch wie $\exp (-\epsilon|x|)$ abklingen läßt, in Halbebenen wohldefiniert, die sich überlappen: Gleichung (B.13) gilt nur in

$$
S_{\oplus}=\{k \in \mathbb{C}: \operatorname{Im} k>-\epsilon\}
$$

Gleichung (B.14) gilt nur in der Halbebene

$$
S_{\ominus}=\{k \in \mathbb{C}: \operatorname{Im} k<+\epsilon\}
$$

Beide Gleichungen gelten in dem Streifen

$$
S_{\oplus} \cap S_{\ominus}=\{k \in \mathbb{C}:-\epsilon<\operatorname{Im} k<+\epsilon\}
$$

Ich kenne weder $\Phi$ noch seine Ableitung in der Nähe von $x=0$, daher sind die Gleichungen (B.13) und (B.14) zunächst ohne Nutzen. Ich kenne aber den Sprung, den diese Größen dort machen, damit das gesamte Feld $\Phi_{\text {ges }}$ und seine Ableitung dort stetig bleibt:

$$
\begin{aligned}
\Phi\left(0^{+}\right)-\Phi\left(0^{-}\right) & =e^{i k_{-} \cdot 0^{-}}=1 \\
\partial_{x} \Phi\left(0^{+}\right)-\partial_{x} \Phi\left(0^{-}\right) & =i k_{-} e^{i k_{-} \cdot 0^{-}}=i k_{-}
\end{aligned}
$$

Mit Kenntnis der Sprungbedingungen bei $x=0$ kann ich die gesuchte WiEnERHopf-Gleichung als Differenz von (B.13) und (B.14) aufstellen, und erhalte schließlich:

$$
\frac{k_{-}^{2}-k^{2}}{k_{+}^{2}-k^{2}} \Phi_{\oplus}+\Phi_{\ominus}=\frac{i\left(k+k_{-}\right)}{k_{+}^{2}-k^{2}}
$$




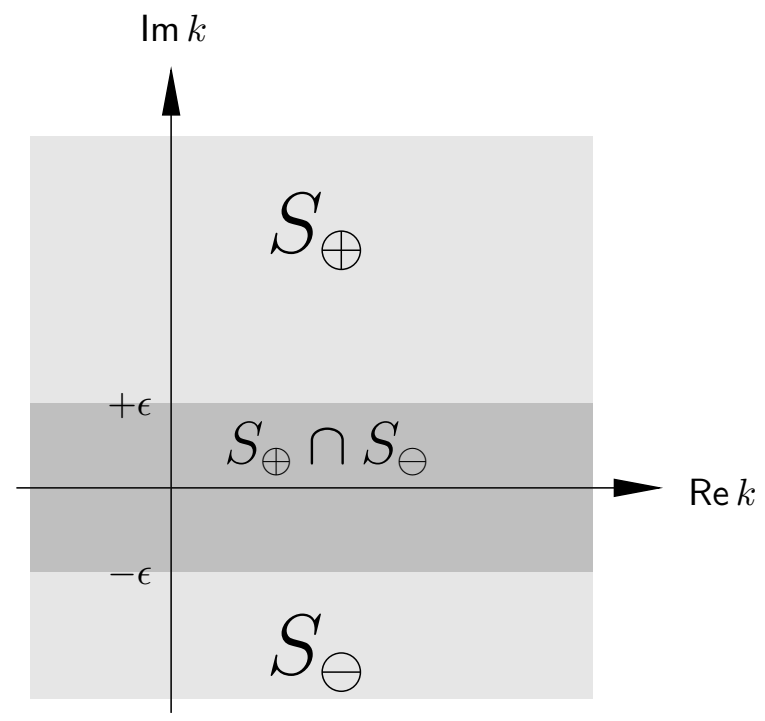

Abbildung B.2: Die Halbebenen $S_{\oplus}$ und $S_{\ominus}$

(B.17) gilt nur, wo (B.13) und (B.14) simultan gültig sind, also in dem 2є-Streifen um die relle Achse.

Jetzt löse ich (B.17), indem ich nach Plus- und Minus-Funktionen separiere:

$\Phi_{\oplus}$ ist eine Plus- und $\Phi_{\ominus}$ eine Minusfunktion.

Den Faktor vor $\Phi_{\oplus}$ muß ich multiplikativ zerlegen:

$$
\mathcal{L} \doteq \frac{k_{-}^{2}-k^{2}}{k_{+}^{2}-k^{2}}=\frac{k_{-}+k}{k_{+}+k} \cdot \frac{k_{-}-k}{k_{+}-k} \doteq \mathcal{L}_{\ominus} \cdot \mathcal{L}_{\oplus}
$$

Wenn ich jetzt durch $\mathcal{L}_{\ominus}$ dividiere, steht auf der linken Seite eine Summe aus einer Plus- und einer Minusfunktion. Um nach solchen zu sortieren, muß ich jetzt noch die rechte Seite additiv zerlegen:

$$
\begin{aligned}
M & \doteq \frac{i\left(k+k_{-}\right)\left(k_{+}-k\right)}{\left(k_{+}^{2}-k^{2}\right)\left(k_{-}-k\right)} \\
& =\frac{i\left(k_{-}-k_{+}\right)}{\left(k_{-}+k_{+}\right)\left(k_{+}+k\right)}+\frac{2 i k_{-}}{\left(k_{-}+k_{+}\right)\left(k_{-}-k\right)} \\
& \doteq M_{\oplus}+M_{\ominus}
\end{aligned}
$$


Jetzt ordne ich Gleichung (B.17), die in den Hilfsgrößen folgende Gestalt hat,

$$
\mathcal{L}_{\oplus} \Phi_{\oplus}+\frac{\Phi_{\ominus}}{\mathcal{L}_{\ominus}}=M_{\oplus}+M_{\ominus}
$$

nach Plus- und Minusfunktionen, und erhalte die separierte WIENER-HopfGleichung

$$
\frac{k_{-}+k}{k_{+}+k} \Phi_{\oplus}-\frac{i\left(k_{-}-k_{+}\right)}{\left(k_{-}+k_{+}\right)\left(k_{+}+k\right)}=\frac{2 i k_{-}}{\left(k_{-}+k_{+}\right)\left(k_{-}-k\right)}-\frac{k_{+}-k}{k_{-}-k} \Phi_{\ominus}=J(k)
$$

in der links nur Plus- und rechts nur Minusfunktionen stehen.

Ich habe damit eine Plusfunktion, holomorph in der oberen Halbebene $S_{\oplus}$, und eine Minusfunktion, holomorph in der unteren Halbebene $S_{\ominus}$, die auf dem Überlappungsgebiet $S_{\ominus} \cap S_{\oplus}$ gleich sind. Daher entsprechen sie einer Funktion $J$, welche auf ganz $\mathbb{C}$ holomorph ist.

$\Phi_{ \pm}(k)$ verhalten sich für $k \rightarrow \infty$ wie $k^{-1}$, denn $\Phi(x)$ bleibt nahe $x=0$ endlich; siehe (B.13) und (B.14).

Wegen (B.20) fällt dann auch $J$ wie $k^{-1}$ ab, dann muß aber schon

$$
J \equiv 0
$$

sein, weil das die einzige auf ganz $\mathbb{C}$ holomorphe, im Unendlichen abklingende Funktion ist. Auf diese Weise werden aus (B.20) zwei Gleichungen:

$$
\begin{aligned}
\Phi_{\oplus} & =\frac{i\left(k_{-}-k_{+}\right)}{\left(k_{-}+k_{+}\right)\left(k_{-}+k\right)} \\
\Phi_{\ominus} & =\frac{2 i k_{-}}{\left(k_{-}+k_{+}\right)\left(k_{+}-k\right)}
\end{aligned}
$$

Um das gesuchte Feld zu finden, muß ich nun noch die Rücktransformation durchführen, wofür der Residuensatz benutzt wird. Abhängig vom Vorzeichen von $x$ muß ich den Integrationsweg unterschiedlich schließen und erhalte Beiträge unterschiedlicher Pole. Das ist die mathematische Repräsentation der Tatsache, daß abhängig vom Vorzeichen von $x$ (links oder rechts der Dichte-Diskontinuität) unterschiedliche Moden im Streufeld vorhanden sind, in unserem Falle mit den Wellenzahlen $k_{-}$beziehungsweise $k_{+}$.

Für das Gebiet $x<0$ erhalte ich so das folgende Streufeld (siehe Abb. B.3): 


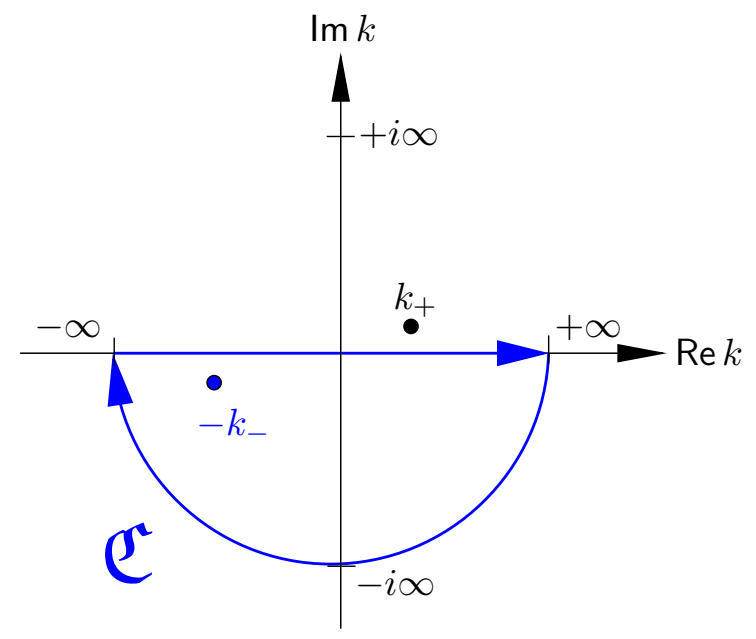

Abbildung B.3: Integrationsweg für $\Phi(x<0)$

$$
\begin{aligned}
\Phi(x) & =\frac{1}{2 \pi} \int_{-\infty}^{+\infty} d k e^{i k x}\left(\Phi_{\oplus}(k)+\Phi_{\ominus}(k)\right) \\
& \stackrel{x \leq 0}{=} \frac{1}{2 \pi} \oint_{\mathfrak{C}} d k e^{i k x}\left(\Phi_{\oplus}(k)+\Phi_{\ominus}(k)\right) \\
& =\frac{1}{2 \pi} \oint_{\mathfrak{C}} d k e^{i k x}\left(\frac{i\left(k_{-}-k_{+}\right)}{\left(k_{-}+k_{+}\right)\left(k_{-}+k\right)}+\frac{2 i k_{-}}{\left(k_{-}+k_{+}\right)\left(k_{+}-k\right)}\right) \\
& =\frac{k_{-}-k_{+}}{k_{+}+k_{-}} e^{-i k_{-} x} \\
& =R_{\leftarrow} e^{-i k_{-} x}
\end{aligned}
$$

Für $x>0$ trägt analog nur $\Phi_{\ominus}(k)$ mit dem Pol $k_{+}$bei:

$$
\Phi(x) \stackrel{x \geq 0}{=} T_{\rightarrow} e^{i k_{+} x}
$$

Die Wiener-Hopf-Methode liefert also das gleiche Ergebnis wie die elementare Rechnung. Sie ist bei einem so einfachen Problem viel aufwendiger als der direkte Ansatz, wird allerdings zum geeigneten Werkzeug, sobald es um unübersichtliche Randwertprobleme geht, wie das bei den Streurechnungen für resonanzartig ausgekleidete Kanalstücke der Fall ist. 


\section{Anhang C}

\section{Zur Berechnung der Streufaktoren}

Die Ergebnisse aus [10] lassen sich auf einen Kanal mit kreisförmigem Querschnitt übertragen. Ich präsentiere hier nur die Ausdrücke, die sich durch die Wahl einer anderen Geometrie verändern, und verweise für die detaillierte Herleitung auf [10].

\section{C.1 Die dimensionslosen Grundgleichungen}

Für das Rohr wähle ich wie üblich Zylinderkoordinaten $x_{0}, r_{0}, \phi_{0}$ wie in Abb. C.1. Die Grundgleichungen behalten ihre Form, lediglich die Differentialoperatoren (LAPLACE-Operator und Gradient) haben in Koordinaten ausgedrückt jetzt eine andere Gestalt, nachzulesen etwa in [12]. Als Referenzlänge bietet sich jetzt statt der Höhe des Rechteckkanals der Rohrdurchmesser $L_{\text {ref }}=2 R_{0}$ an. Wie in [10] bezeichnen Ausdrücke ohne den Subscript 0 dimensionslose Größen.

Gleichung (2) wird so zu

$$
\left(\frac{\partial^{2}}{\partial x^{2}}+\frac{1}{r} \frac{\partial}{\partial r} r \frac{\partial}{\partial r}+\frac{1}{r^{2}} \frac{\partial^{2}}{\partial \phi^{2}}+K^{2}\right) \Phi=0
$$

Weil die Auskleidung nicht mehr nur zwei gegenüberliegende Wandabschnitte, sondern den ganzen Umfang des Meßobjektes umfaßt, lauten die Gleichungen (4) nun

$$
\frac{\partial \Phi}{\partial r}(x, R, \phi)=0, \quad x<0 \text { oder } x>l
$$



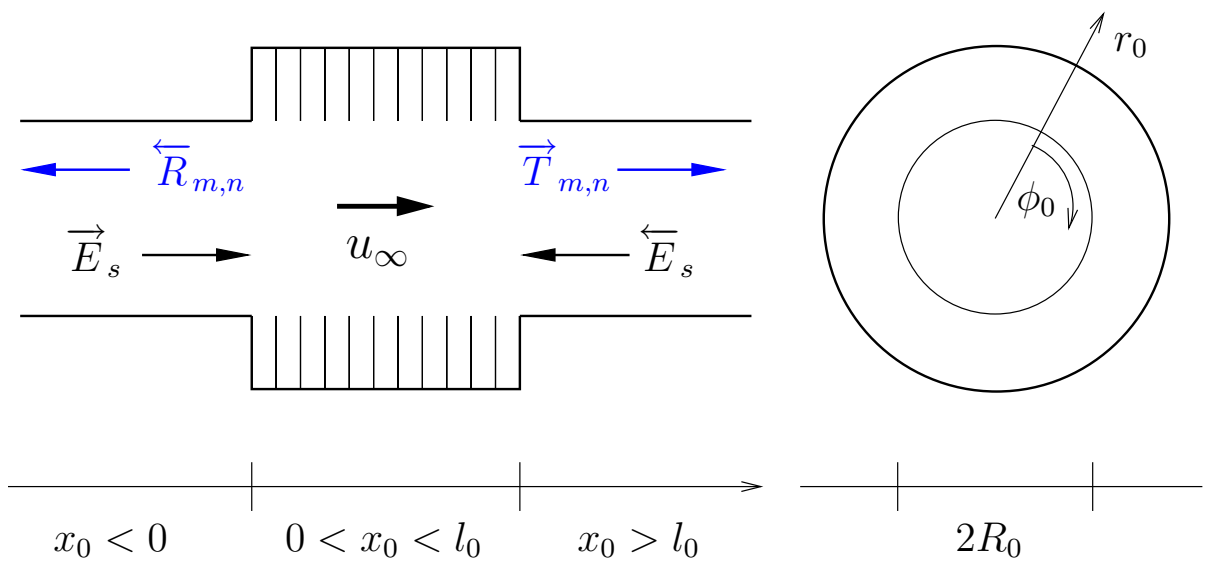

Abbildung C.1: Bezeichnungen und Koordinaten für die Rohrgeometrie

Bei Einführung der Wirbelschicht-Auslenkung $\eta$ ist zu beachten, daß diese nicht mehr getrennt auf zwei Zusammenhangskomponenten $z=0, h$, sondern über den gesamten Mantel definiert ist. Positive Werte für $\eta$ sollen dabei Auslenkungen nach außen, also in die Kammern hinein, beschreiben. Gleichungen (8) und (9) lauten daher

$$
\begin{array}{cc}
\frac{\partial \Phi}{\partial r}(x, R, \phi)=\frac{i K}{M a \sqrt{1-M a^{2}}}\left(1+\frac{M a}{i K} \frac{\partial}{\partial x}\right) \eta(x, R, \phi), & 0<x<l \\
\eta(x, R, \phi)=-\frac{M a}{Z\left(1-M a^{2}\right)}\left(1+\frac{M a}{i K} \frac{\partial}{\partial x}\right) \Phi(x, R, \phi), & 0<x<l
\end{array}
$$

Analog zu [10] spalte ich als nächstes das gesuchte Feld nach Eigenfunktionen zum winkelabhängigen Teil des LAPLACE-Operators auf,

$$
\Phi(x, r, \phi)=\sum_{m=-\infty}^{\infty} \Phi_{m}(x, r) e^{i m \phi}
$$

betrachte im folgenden nur noch das Problem für festes $m$ und erhalte anstelle von (10) folgende Differentialgleichung:

$$
\left(\frac{\partial^{2}}{\partial x^{2}}+\frac{1}{r} \frac{\partial}{\partial r} r \frac{\partial}{\partial r}+K^{2}-\frac{m^{2}}{r^{2}}\right) \Phi_{m}=0
$$


Als nächsten Schritt separieren die Autoren die ausbreitungsfähigen Wellen der schallharten Teilrohre, um für die weitere Analyse nur noch mit einem FourIERtransformierbaren (Nah-)Feld arbeiten zu können. Die entsprechenden Eigenfunktionen zu Gleichung C.6 enthalten jetzt allerdings keine Cosinus- sondern Bessel-Funktionen. Für $x<0$ lautet diese Zerlegung

$$
\begin{aligned}
\Phi_{m}(x, r)= & \vec{E}_{m, s} e^{i \gamma_{m, s} x} J_{m}\left(K_{s}^{m} r\right)+\sum_{n=0}^{N(m)} \overleftarrow{R}_{m, n} e^{-i \gamma_{m, n} x} J_{m}\left(K_{n}^{m} r\right) \\
& +\phi_{m}(x, r)
\end{aligned}
$$

und für $x>l$

$$
\begin{aligned}
\Phi_{m}(x, r)= & \overleftarrow{E}_{m, s} e^{-i \gamma_{m, s} x} J_{m}\left(K_{s}^{m} r\right)+\sum_{n=0}^{N(m)} \vec{T}_{m, n} e^{i \gamma_{m, n} x} J_{m}\left(K_{n}^{m} r\right) \\
& +\psi_{m}(x, r)
\end{aligned}
$$

Die Wellenzahlen $\gamma_{m, n}$ und $K_{n}^{m}$ sind, analog zu Gleichung (13) der Arbeit [10] so definiert, daß die Differentialgleichung (C.6) und die schallharte Randbedingung (C.2) erfüllt werden:

$$
\begin{gathered}
K_{n}^{m} J_{m}^{\prime}\left(K_{n}^{m} R\right)=0 \\
\gamma_{m, n}= \begin{cases}-\sqrt{K^{2}-\left(K_{n}^{m}\right)^{2}} & K^{2}>\left(K_{n}^{m}\right)^{2} \\
i \sqrt{\left(K_{n}^{m}\right)^{2}-K^{2}} & K^{2}<\left(K_{n}^{m}\right)^{2}\end{cases}
\end{gathered}
$$

Die natürliche Zahl $N(m)$ ist die Ordnung der höchsten noch ausbreitungsfähigen Mode, also die größte Zahl, für die noch

$$
k^{2}>\left(K_{N(m)}^{m}\right)^{2}
$$

gilt. Innerhalb der von BRANDES vermessenen Frequenzen ist sie stets Null: Alle höheren Moden sind gedämpft.

Die von den Autoren eingeführten unbekannten druck- und ausklenkungsartigen Funktionen (Gleichungen (14) und (15)) müssen nicht nach oberer und unte- 
rer Wand unterschieden werden, da die Auskleidung nun zusammenhängend ist. Insofern ist die zylindrische Geometrie sogar übersichtlicher:

$$
\begin{aligned}
& \frac{\partial \phi_{m}}{\partial r}(x, R)=\left\{\begin{array}{cl}
0 & , x<0 \\
\frac{i K}{M a \sqrt{1-M a^{2}}}\left(1+\frac{M a}{i K} \frac{\partial}{\partial x}\right) \eta_{m}(x) & , 0<x<l \\
0 & , x>l
\end{array}\right. \\
& \frac{M a}{Z\left(1-M a^{2}\right)}\left(1+\frac{M a}{i K} \frac{\partial}{\partial x}\right) \phi_{m}(x, R)= \\
& \left\{\begin{array}{cl}
g_{-}(x) & , x<0 \\
-\eta_{m}(x)-\frac{M a}{Z\left(1-M a^{2}\right)} R_{m}(x) & , 0<x<l \\
g_{+}(x)-\frac{M a}{Z\left(1-M a^{2}\right)}\left(R_{m}(x)-T_{m}(x)\right) & , x>l
\end{array}\right.
\end{aligned}
$$

Die Hilfsgrößen $R_{m}, T_{m}$ enthalten nun ebenfalls BEsSEL-Funktionen:

$$
\begin{aligned}
R_{m}(x) & =\left(1+\frac{M a}{K} \gamma_{m, s}\right) \vec{E}_{m, s} e^{i \gamma_{m, s} x} J_{m}\left(K_{s}^{m} R\right) \\
& +\sum_{n=0}^{N(m)}\left(1-\frac{M a}{K} \gamma_{m, n}\right) \overleftarrow{R}_{m, n} e^{-i \gamma_{m, n} x} J_{m}\left(K_{n}^{m} R\right)
\end{aligned}
$$

und für $x>l$

$$
\begin{aligned}
T_{m}(x) & =\left(1-\frac{M a}{K} \gamma_{m, s}\right) \overleftarrow{E}_{m, s} e^{-i \gamma_{m, s} x} J_{m}\left(K_{s}^{m} R\right) \\
& +\sum_{n=0}^{N(m)}\left(1+\frac{M a}{K} \gamma_{m, n}\right) \vec{T}_{m, n} e^{i \gamma_{m, n} x} J_{m}\left(K_{n}^{m} R\right)
\end{aligned}
$$

\section{C.2 Aufstellen der WIEnER-Hopf-Gleichung}

Der nächste Schritt besteht darin, alle relevanten Gleichungen bezüglich $x$ zu FouRIER-transformieren und daraus die WIENER-Hopf-Gleichung als Bestimmungsgleichung für die unbekannten Funktionen $\eta_{m}$ und $g_{ \pm}$aufzustellen. 
Die Differentialgleichung (C.6) wird dabei (die Variable im FouRIER-Raum bezeichne ich wie in [10] mit $\xi) \mathrm{zu}$

$$
\left(\frac{1}{r} \frac{\partial}{\partial r} r \frac{\partial}{\partial r}+K^{2}-\xi^{2}-\frac{m^{2}}{r^{2}}\right) \phi_{m}(\xi, r)=0
$$

so daß statt Gleichung (16) in der Arbeit [10] der Ansatz nun lautet:

$$
\begin{aligned}
\phi_{m}(\xi, r) & =\alpha_{m}(\xi) J_{m}(\gamma(\xi) r) \\
\gamma(\xi) & =\sqrt{K^{2}-\xi^{2}}
\end{aligned}
$$

In der etwas abweichenden Definition von $\gamma$ habe ich die in [10] vorkommende Umdefinition (dort im Anschluß an Gleichung (22)) vorweggenommen.

Die FourIER-Transformation von (C.11)-(C.14) ergibt

$$
\begin{aligned}
& \frac{\partial \phi_{m}}{\partial r}(\xi, R)=\frac{i K}{M a \sqrt{1-M a^{2}}}\left(1+\frac{M a}{K} \xi\right) \eta_{m}(\xi) \\
& +\frac{1}{\sqrt{1-M a^{2}}}\left(\eta_{m}(l) e^{-i \xi l}-\eta_{m}(0)\right) \\
& \frac{M a}{Z\left(1-M a^{2}\right)}\left(1+\frac{M a}{K} \xi\right) \phi_{m}(\xi, R) \\
& =g_{\oplus}(\xi)-\eta_{m}(\xi)+e^{-i \xi l} g_{\ominus}(\xi) \\
& -\frac{M a}{Z\left(1-M a^{2}\right)}\left(R_{m}(\xi)-e^{-i \xi l} T_{m}(\xi)\right) \\
& R_{m}(\xi)=\left(1+\frac{M a}{K} \gamma_{m, s}\right) \frac{\vec{E}_{m, s}}{i\left(\xi-\gamma_{m, s}\right)} J_{m}\left(K_{s}^{m} R\right) \\
& +\sum_{n=0}^{N(m)}\left(1-\frac{M a}{K} \gamma_{m, n}\right) \frac{\overleftarrow{R}_{m, n}}{i\left(\xi+\gamma_{m, n}\right)} J_{m}\left(K_{n}^{m} R\right)
\end{aligned}
$$




$$
\begin{aligned}
T_{m}(\xi) & =\left(1-\frac{M a}{K} \gamma_{m, s}\right) \frac{\overleftarrow{E}_{m, s} e^{-i \gamma_{m, s} l}}{i\left(\xi+\gamma_{m, s}\right)} J_{m}\left(K_{s}^{m} R\right) \\
& +\sum_{n=0}^{N(m)}\left(1+\frac{M a}{K} \gamma_{m, n}\right) \frac{\vec{T}_{m, n} e^{i \gamma_{m, n} l}}{\left.\xi-\gamma_{m, n}\right)} J_{m}\left(K_{n}^{m} R\right)
\end{aligned}
$$

Der Ansatz und die beiden Randbedingungen können nun analog zu den Gleichungen (20)-(22) zu einer einzigen Gleichung für die drei unbekannten Funktionen $\eta(\xi), g_{\oplus}(\xi), g_{\ominus}(\xi)$ kombiniert werden. Das Ergebnis, die WIENER-HopfGleichung für das durchströmte Rohr, hat die Gestalt

$$
\begin{array}{r}
g_{\oplus}(\xi)-\mathcal{L}(\xi) \eta_{m}(\xi)+e^{-i \xi l} g_{\ominus}(\xi)=\frac{M a}{Z\left(1-M a^{2}\right)}\left(R_{m}(\xi)-e^{-i \xi l} T_{m}(\xi)\right) \\
-\frac{M a}{Z{\sqrt{1-M a^{2}}}^{3}}\left(\eta_{m}(0)-\eta_{m}(l) e^{-i \xi l}\right) \frac{J_{m}(\gamma R)}{\gamma J_{m}^{\prime}(\gamma R)}
\end{array}
$$

und die für die weitere Analyse zentrale Funktion $\mathcal{L}$ sieht dabei wie folgt aus:

$$
\mathcal{L}(\xi)=1+\frac{i K\left(1+\frac{M a}{K} \xi\right)^{2} J_{m}(\gamma R)}{Z{\sqrt{1-M a^{2}}}^{3} \gamma J_{m}^{\prime}(\gamma R)}
$$

Die Unterscheidung zwischen symmetrischer und antisymmetrischer Anregung fällt aus den bereits genannten Gründen weg; es gibt nur eine einzige Gleichung ${ }^{1}$.

\section{C.3 Zerlegen der WIENER-HOPF-Gleichung}

Als nächstes wird in [10] die WIEnER-Hopf-Gleichung in Plus- und MinusFunktionen zerlegt, um aus ihr Bestimmungsgleichungen für $\eta(\xi), g_{\oplus}(\xi)$ und $g_{\ominus}(\xi)$ herleiten zu können. $\mathcal{L}$ muß dabei-ähnlich wie in Anhang B beschriebenmultiplikativ zerlegt werden. Ich wähle die von den Autoren favorisierte kausale Aufspaltung, bei der die instabile Mode im Stromab-Sektor (in $\mathcal{L}_{\ominus}$ ) berücksichtigt wird. Im Stromauf-Anteil $\mathcal{L}_{\oplus}$ sind daher nur noch die herkömmlichen StromaufModen enthalten, die ich mit $\lambda_{m, n}^{-}, n=0,1, \ldots$ bezeichne, während in $\mathcal{L}_{\ominus}$ neben

\footnotetext{
${ }^{1}$ Der „,antisymmetrische“ Fall ist im Rohr in den Moden zu $m= \pm 1$ verwirklicht.
} 
den herkömmlichen Stromab-Moden die instabile $\left(\lambda_{m}^{\text {instab }}\right)$ und die gedämpfte hydrodynamische $(n=-1)$ Welle enthalten sind:

$$
\begin{aligned}
& \mathcal{L}_{\oplus}(\xi)=\mathcal{L}(0) \prod_{n=0}^{\infty} \frac{1+\xi / \lambda_{m, n}^{-}}{1+\xi / \gamma_{m, n}} \\
& \mathcal{L}_{\ominus}(\xi)=\left(1+\frac{\xi}{\lambda_{m}^{\text {instab }}}\right)\left(1-\frac{\xi}{\lambda_{m,-1}^{+}}\right) \prod_{n=0}^{\infty} \frac{1-\xi / \lambda_{m, n}^{+}}{1-\xi / \gamma_{m, n}}
\end{aligned}
$$

Die möglicherweise verwirrende Vorzeichenkonvention von [10], nach der die Nullstellen von $\mathcal{L}$ mit $\lambda_{m, n}^{+},-\lambda_{m, n}^{-}$und $-\lambda_{m}^{\text {instab }}$ bezeichnet werden, behalte ich hier der Vergleichbarkeit wegen bei. Für Gleichung C.24 und vergleichbare Summen werde ich die Schreibweise

$$
\mathcal{L}_{\ominus}(\xi)=\frac{\prod_{n=-2}^{\infty}\left(1-\xi / \lambda_{m, n}^{+}\right)}{\prod_{n=0}^{\infty}\left(1-\xi / \gamma_{m, n}\right)}
$$

benutzen; in diesen Ausdrücken gilt also für den ersten Summanden

$$
\lambda_{m,-2}^{+} \doteq-\lambda_{m}^{\text {instab }}
$$

Weil zwei Diskontinuitäten (bei $x=0$ und $x=l$ ) vorhanden sind, gibt es zwei mögliche Zerlegungen der Gleichung (C.21). Die erste geht aus (C.21) durch Multiplikation mit $1 / \mathcal{L}_{\oplus}$, die zweite durch Multiplikation mit $\exp (i \xi l) / \mathcal{L}_{\ominus}$ hervor. Koch, MöHRING nehmen nach der Separation an, daß die in ganz $\mathbb{C}$ holomorphen Funktionen $J_{i}(\xi), i=1,2$, identisch verschwinden. Auf diese Weise erhalten sie vier Gleichungen für $g_{\oplus}, g_{\ominus}, \eta_{m}$ und $\exp (i \xi l) \eta_{m}$.

Für die Rohrgeometrie hat die FouRIER-Transformierte der druckartigen Funktion $g_{-}$die folgende Gestalt: 


$$
\begin{aligned}
g_{\oplus}=-\mathcal{L}_{\oplus} & \left\{\sum_{n=0}^{\infty} \frac{P_{n}\left(-\lambda_{m, n}^{-}\right)}{\xi+\lambda_{m, n}^{-}} u_{\ominus}^{*}\left(-\lambda_{m, n}^{-}\right)\right. \\
& -\frac{M a}{Z\left(1-M a^{2}\right)}\left(\frac{R_{m}}{\mathcal{L}_{\oplus}}-\frac{\left(1+\gamma_{m, s} \frac{M a}{K}\right) \vec{E}_{m, s} J_{m}\left(K_{s}^{m} R\right)}{i\left(\xi-\gamma_{m, s}\right) \mathcal{L}_{\oplus}\left(\gamma_{m, s}\right)}\right) \\
& -\frac{\eta_{m}(0)}{i(\xi+K / M a)}\left(\frac{1}{\mathcal{L}_{\oplus}}-\frac{1}{\mathcal{L}_{\oplus}(-K / M a)}\right) \\
& \left.+\eta_{m}(l) \sum_{n=0}^{\infty} \frac{R_{n}\left(-\lambda_{m, n}^{-}\right)}{\xi+\lambda_{m, n}^{-}}\right\}
\end{aligned}
$$

Für die Transformierte von $g_{+}$erhalte ich den folgenden Ausdruck (die Summen laufen jetzt über die beiden zusätzlichen Glieder für die hydrodynamischen Moden):

$$
\begin{aligned}
g_{\ominus}=\mathcal{L}_{\ominus} & \left\{-\sum_{n=-2}^{\infty} \frac{Q_{n}\left(\lambda_{m, n}^{+}\right)}{\xi-\lambda_{m, n}^{+}} u_{\oplus}^{*}\left(\lambda_{m, n}^{+}\right)\right. \\
& -\frac{M a}{Z\left(1-M a^{2}\right)}\left(\frac{T_{m}}{\mathcal{L}_{\ominus}}-\frac{\left(1-\gamma_{m, s} \frac{M a}{K}\right) e^{-i \gamma_{m, s}} \overleftarrow{E}_{m, s} J_{m}\left(K_{s}^{m} R\right)}{i\left(\xi+\gamma_{m, s}\right) \mathcal{L}_{\ominus}\left(-\gamma_{m, s}\right)}\right) \\
& +\eta_{m}(0) \sum_{n=-2}^{\infty} \frac{S_{n}\left(\lambda_{m, n}^{+}\right)}{\xi-\lambda_{m, n}^{+}} \\
& \left.-\frac{\eta_{m}(l)}{i(\xi+K / M a)}\left(\frac{1}{\mathcal{L}_{\ominus}}-\frac{1}{\mathcal{L}_{\ominus}(-K / M a)}\right)\right\}
\end{aligned}
$$

Die FouRIER-Transformierte der Auslenkungsfunktion $\eta_{m}$ erfüllt folgende Gleichung $^{2}$ :

\footnotetext{
${ }^{2}$ Die vierte Gleichung für $\exp (i \xi l) \eta_{m}$ ist äquivalent.
} 


$$
\begin{aligned}
& \eta_{m}= \frac{1}{\mathcal{L}_{\ominus}}\left\{-\sum_{n=0}^{\infty} \frac{P_{n}\left(-\lambda_{m, n}^{-}\right)}{\xi+\lambda_{m, n}^{-}} u_{\ominus}^{*}\left(-\lambda_{m, n}^{-}\right)-\frac{M a\left(1+\gamma_{m, s} \frac{M a}{K}\right) \vec{E}_{m, s} J_{m}\left(K_{s}^{m} R\right)}{i Z\left(1-M a^{2}\right)\left(\xi-\gamma_{m, s}\right) \mathcal{L}_{\oplus}\left(\gamma_{m, s}\right)}\right. \\
&\left.+\frac{\eta_{m}(0)}{i(\xi+K / M a)}\left(\mathcal{L}_{\ominus}-\frac{1}{\mathcal{L}_{\oplus}(-K / M a)}\right)-\eta_{m}(l) \sum_{n=0}^{\infty} \frac{R_{n}\left(-\lambda_{m, n}^{-}\right)}{\xi+\lambda_{m, n}^{-}}\right\} \\
&+\frac{e^{-i \xi l}}{\mathcal{L}_{\oplus}}\left\{-\sum_{n=-2}^{\infty} \frac{Q_{n}\left(\lambda_{m, n}^{+}\right)}{\xi-\lambda_{m, n}^{+}} u_{\oplus}^{*}\left(\lambda_{m, n}^{+}\right)+\frac{M a\left(1-\gamma_{m, s} \frac{M a}{K}\right) e^{-i \gamma_{m, s} l} \overleftarrow{E}_{m, s} J_{m}\left(K_{s}^{m} R\right)}{i Z\left(1-M a^{2}\right)\left(\xi+\gamma_{m, s}\right) \mathcal{L}_{\ominus}\left(-\gamma_{m, s}\right)}\right. \\
&\left.+\eta_{m}(0) \sum_{n=-2}^{\infty} \frac{S_{n}\left(\lambda_{m, n}^{+}\right)}{\xi-\lambda_{m, n}^{+}}-\frac{\eta_{m}(l)}{i(\xi+K / M a)}\left(\mathcal{L}_{\oplus}-\frac{1}{\mathcal{L}_{\ominus}(-K / M a)}\right)\right\}
\end{aligned}
$$

Analog zu [10] habe ich dabei die folgenden Hilfsfunktionen eingeführt:

$$
\begin{aligned}
& u_{\ominus}^{*}=\frac{1}{\mathcal{L}_{\ominus}}\left(g_{\ominus}+\frac{M a}{Z\left(1-M a^{2}\right)} T_{m}\right) \\
& u_{\oplus}^{*}=\frac{1}{\mathcal{L}_{\oplus}}\left(g_{\oplus}-\frac{M a}{Z\left(1-M a^{2}\right)} R_{m}\right)
\end{aligned}
$$

sowie die Ausdrücke 


$$
\begin{aligned}
& P_{n}\left(-\lambda_{m, n}^{-}\right)=e^{i \lambda_{m, n}^{-} l}\left(\mathcal{L}_{\ominus}\left(-\lambda_{m, n}^{-}\right)\right)^{2}\left(K^{2}-\left(\lambda_{m, n}^{-}\right)^{2}\right) / \\
& \left(\lambda_{m, n}^{-}+\frac{i K R \lambda_{m, n}^{-}\left(1-\lambda_{m, n}^{-} \frac{M a}{K}\right)^{2}}{Z{\sqrt{1-M a^{2}}}^{3}}\right. \\
& +\frac{Z{\sqrt{1-M a^{2}}}^{3} \lambda_{m, n}^{-}\left((K R)^{2}-\left(\lambda_{m, n}^{-} R\right)^{2}-m^{2}\right)}{i K R\left(1-\lambda_{m, n}^{-} \frac{M a}{K}\right)^{2}} \\
& \left.-\frac{2\left(K^{2}-\left(\lambda_{m, n}^{-}\right)^{2}\right) \frac{M a}{K}}{1-\lambda_{m, n}^{-} \frac{M a}{K}}\right) \\
& R_{n}\left(-\lambda_{m, n}^{-}\right)=\frac{M a}{i K} \frac{P_{2 n}\left(-\lambda_{m, n}^{-}\right)}{\mathcal{L}_{\ominus}\left(-\lambda_{m, n}^{-}\right)\left(1-\lambda_{m, n}^{-} \frac{M a}{K}\right)} \\
& Q_{n}\left(\lambda_{m, n}^{+}\right)=-e^{i \lambda_{m, n}^{+} l}\left(\mathcal{L}_{\oplus}\left(\lambda_{m, n}^{+}\right)\right)^{2}\left(K^{2}-\left(\lambda_{m, n}^{+}\right)^{2}\right) / \\
& \left(\lambda_{m, n}^{+}+\frac{i K R \lambda_{m, n}^{+}\left(1+\lambda_{m, n}^{+} \frac{M a}{K}\right)^{2}}{Z{\sqrt{1-M a^{2}}}^{3}}\right. \\
& +\frac{Z{\sqrt{1-M a^{2}}}^{3} \lambda_{m, n}^{+}\left((K R)^{2}-\left(\lambda_{m, n}^{+} R\right)^{2}-m^{2}\right)}{i K R\left(1+\lambda_{m, n}^{+} \frac{M a}{K}\right)^{2}} \\
& \left.+\frac{2\left(K^{2}-\left(\lambda_{m, n}^{+}\right)^{2}\right) \frac{M a}{K}}{1+\lambda_{m, n}^{+} \frac{M a}{K}}\right) \\
& S_{n}\left(\lambda_{m, n}^{+}\right)=\frac{M a}{i K} \frac{Q_{n}\left(\lambda_{m, n}^{+}\right)}{\mathcal{L}_{\oplus}\left(\lambda_{m, n}^{+}\right)\left(1+\lambda_{m, n}^{+} \frac{M a}{K}\right)}
\end{aligned}
$$

Wertet man die Gleichung (C.25) bei $\xi=\lambda_{m, n}^{+}$und (C.26) bei $\xi=-\lambda_{m, n}^{-}$aus und verwendet noch einmal die Definitionen (C.28), dann ergibt sich schließlich ein lineares Gleichungssystem für die unbekannten Hilfsgrößen $u_{\oplus}^{*}\left(\lambda_{m, n}^{+}\right), u_{\ominus}^{*}\left(-\lambda_{m, n}^{-}\right)$.

\section{C.4 Sprungbedingungen}

Zur Lösung werden die Werte $\eta_{m}(0), \eta_{m}(l)$ der Wirbelschicht-Auslenkung benötigt. 
In [10] wird aus dem asymptotischen Verhalten der Fouriertransformierten der Auslenkungsfunktion gefolgert, daß am Stromauf-Ende für die Auslenkung die KUTTA-Bedingung erfüllt ist, also Funktionswert und Ableitung nach $x$ verschwinden. Der Wert am Stromab-Ende wird dagegen formal unendlich, ist allerdings distributionstheoretisch zu kontrollieren. Wie dies geschieht und mit welcher Wahl für $\eta_{m}(l)$ die Autoren auf die dann folgenden numerischen Ergebnisse kommen, wird allerdings nicht mitgeteilt.

In den Gleichungen (C.25)-(C.27) sind die gesuchten Funktionen $g_{\oplus}, g_{\ominus}$ und $\eta_{m}$ durch die schon eingeführten Hilfsgrößen ausgedrückt. Analog zu [10] verhalten sich die Splitfunktionen von $\mathcal{L}(\xi)$ für $|\xi| \rightarrow \infty$ asymptotisch wie

$$
\begin{aligned}
& \mathcal{L}_{\oplus} \sim \xi^{-\frac{1}{2}} \\
& \mathcal{L}_{\ominus} \sim \xi^{+\frac{3}{2}}
\end{aligned}
$$

Die Asymptotik der anderen Größen läßt sich direkt ablesen. Das versetzt mich in die Lage, Aussagen über die Werte der Funktionen $g_{-}, g_{+}$und $\eta_{m}$ bei $x=0$ beziehungsweise $x=l$ zu treffen, denn es gilt folgender Satz (siehe [5]):

Sei $f(\xi)$ die Laplace-Transformierte von $f(x)$. Wenn $f$ nahe $x=0$ durch die Reihe

$$
f(x)=\sum_{i=0}^{\infty} c_{i} x^{\nu_{i}}
$$

mit den Exponenten $-1<\nu_{0}<\nu_{1}<\ldots$ darstellbar ist, so besitzt $f(\xi)$ für $\xi \rightarrow \infty$ die asymptotische Entwicklung

$$
f(\xi) \sim \sum_{i=0}^{\infty} c_{i} \Gamma\left(\nu_{i}+1\right) \xi^{-\nu_{i}-1}
$$

Als erstes untersuche ich $g_{\oplus}(\xi)$, die Transformierte der druckartigen Funktion $g_{-}(x)$. Sie hat im allgemeinen das asymptotisches Verhalten

$$
g_{\oplus} \sim \xi^{-1}
$$

es sei denn, die Gleichung 


$$
\begin{aligned}
\eta_{m}(0)+\frac{M a}{Z\left(1-M a^{2}\right)}\left(1+\gamma_{m, s} \frac{M a}{K}\right) \vec{E}_{m, s} J_{m}\left(K_{s}^{m} R\right) \\
+\frac{M a}{Z\left(1-M a^{2}\right)} \sum_{n=0}^{N(m)}\left(1-\gamma_{m, n} \frac{M a}{K}\right) \overleftarrow{R}_{m, n} J_{m}\left(K_{n}^{m} R\right)=0
\end{aligned}
$$

ist erfüllt. Dann nämlich verhält sich $g_{\oplus}$ wie

$$
g_{\oplus} \sim \xi^{-\frac{3}{2}}
$$

für große $\xi$.

Wenn $g_{-}(0)$ also endlich ist $\left(\nu_{0}=0\right)$, dann wächst $g_{\oplus}$ wie $\xi^{-1}$ und $g_{-}(0)$ kann durch ein geeignet gewähltes $\eta_{m}(0)$ auf einen bestimmten Wert eingestellt werden, etwa auf Null, wenn die Gleichung (C.36) erfüllt wird.

$g_{\ominus}$, die Transformierte zu $g_{+}(x)$, verhält sich asymptotisch wie

$$
g_{\ominus} \sim \xi^{\frac{1}{2}}
$$

es sei denn, die Gleichung

$$
\begin{array}{r}
\sum_{n=-2}^{\infty} Q_{n}\left(\lambda_{m, n}^{+}\right) u_{\oplus}^{*}\left(\lambda_{m, n}^{+}\right)-\frac{M a\left(1-\gamma_{m, s} \frac{M a}{K}\right) e^{-i \gamma_{m, s} l} \overleftarrow{E}_{m, s} J_{m}\left(K_{s}^{m} R\right)}{i Z\left(1-M a^{2}\right) \mathcal{L}_{\ominus}\left(-\gamma_{m, s}\right)} \\
-\eta_{m}(0) \sum_{n=-2}^{\infty} S_{n}\left(\lambda_{m, n}^{+}\right)-\frac{\eta_{m}(l)}{i \mathcal{L}_{\ominus}\left(-\frac{K}{M a}\right)}=0
\end{array}
$$

ist erfüllt, denn dann fällt $g_{\ominus}$ wie

$$
g_{\ominus} \sim \xi^{-1}
$$

und $g_{+}(l)$ ist endlich, wobei der Wert von $\eta_{m}(l)$ abhängt. Ist die Gleichung nicht erfüllt, kann $g_{+}(l)$ unendlich sein.

Die Transformierte

$$
\eta_{m}(\xi)=\frac{1}{\mathcal{L}_{\ominus}(\xi)}(\ldots)+\frac{e^{-i \xi l}}{\mathcal{L}_{\oplus}(\xi)}(\ldots)
$$


ist eine ganze Funktion. Bei der Rücktransformation kann man deswegen den Integrationsweg deformieren, ohne die Residuen irgendwelcher Pole berücksichtigen zu müssen.

Wähle ich für die Berechnung von $\eta_{m}(0)$ als Integrationsweg einen unendlich fernen Halbkreis in der unteren Halbebene, dann trägt der Summand mit dem Vorfaktor $\exp (-i \xi l) / \mathcal{L}_{\oplus}$ nichts bei-siehe dazu Gleichung (C.27) - und $\eta_{m}(0)$ wird vom asymptotischen Verhalten des ersten Summanden bestimmt. Umgekehrt liefert bei $\eta_{m}(l)$ nur der zweite Summand einen Beitrag, da hier $\eta_{m}(\xi) \exp (i \xi l)$ entlang eines unendlich fernen Halbkreises in der oberen Halbebene integriert werden kann.

Der erste Summand wächst für große $\xi$ wie

$$
\frac{1}{\mathcal{L}_{\ominus}(\xi)}(\ldots) \sim \xi^{-1}
$$

es sei denn,

$$
\eta_{m}(0)=0
$$

Dann fällt der Term sogar mit

$$
\frac{1}{\mathcal{L}_{\ominus}(\xi)}(\ldots) \sim \xi^{-\frac{5}{2}}
$$

$\eta_{m}(0)$ ist also in jedem Falle endlich, wenn es verschwindet, dann verschwindet gemäß des oben zitierten Satzes auch $\partial_{x} \eta_{m}(0)$, so daß die Wirbelschicht tangential am Kontakt schallharte Wand-nachgiebige Wand ablöst.

Der zweite Summand fällt hingegen nur wie

$$
\frac{1}{\mathcal{L}_{\oplus}(\xi)}(\ldots) \sim \xi^{-\frac{1}{2}}
$$

ab, weswegen im allgemeinen $\eta_{m}(l)$ unendlich ist, es sei denn, die schon zitierte Gleichung (C.39) ist erfüllt. Dann nämlich verhält sich dieser Teil wie

$$
\frac{1}{\mathcal{L}_{\oplus}(\xi)}(\ldots) \sim \xi^{-1}
$$

und $\eta_{m}(l)$ ist endlich.

Vom mathematischen Standpunkt aus sind also mehrere Wahlen von $\eta_{m}(0)$ und $\eta_{m}(l)$ möglich: Sie können auf Null oder so gesetzt werden, daß die Gleichun- 
gen (C.36) oder (C.39) erfüllt sind. Welche Wahl von der Natur realisiert wird, läßt sich im Rahmen des Modells selbst nicht beantworten.

\section{C.5 Ausdrücke für die Streufaktoren}

Die Streufaktoren $\vec{T}_{m, \sigma}, \overleftarrow{R}_{m, \sigma}$ ermitteln die Autoren, indem sie a posteriori sicherstellen, daß die Funktionen $g_{\oplus}, g_{\ominus}$ in ihren jeweiligen Halbebenen tatsächlich holomorph sind, was bei der ganzen Analyse vorausgesetzt wurde. Das ist der Fall, wenn die Residuen

$$
\begin{aligned}
\lim _{\xi \rightarrow-\gamma_{m, \sigma}}\left[\left(\xi+\gamma_{m, \sigma}\right) g_{\oplus}(\xi)\right] & =0 \\
\lim _{\xi \rightarrow+\gamma_{m, \sigma}}\left[\left(\xi-\gamma_{m, \sigma}\right) g_{\ominus}(\xi)\right] & =0
\end{aligned}
$$

verschwinden. Mit Hilfe der Definitionen (C.28) lassen sich so die Streufaktoren durch die Hilfsgrößen $u_{\oplus}^{*}, u_{\ominus}^{*}$ ausdrücken. Im Falle der Rohrgeometrie lauten die Formeln schließlich:

$$
\begin{aligned}
& \vec{T}_{m, \sigma}=\frac{-e^{-i \gamma_{m, \sigma} l}\left(\gamma_{m, \sigma}+\frac{K}{M a}\right)}{\sqrt{1-M a^{2}} J_{m}\left(K_{\sigma}^{m} R\right) \mathcal{L}_{\oplus}\left(\gamma_{m, \sigma}\right)} \Delta_{m, \sigma} \times \\
&\left(\frac{M a\left(1-\gamma_{m, s} \frac{M a}{K}\right) e^{-i \gamma_{m, s} l} \overleftarrow{E}_{m, s} J_{m}\left(K_{s}^{m} R\right)}{i Z\left(1-M a^{2}\right)\left(\gamma_{m, \sigma}+\gamma_{m, s}\right) \mathcal{L}_{\ominus}\left(-\gamma_{m, s}\right)}-\sum_{n=-2}^{\infty} \frac{Q_{n}\left(\lambda_{m, n}^{+}\right)}{\gamma_{m, \sigma}-\lambda_{m, n}^{+}} u_{\oplus}^{*}\left(\lambda_{m, n}^{+}\right)\right. \\
&\left.\quad+\eta_{m}(0) \sum_{n=-2}^{\infty} \frac{S_{n}\left(\lambda_{m, n}^{+}\right)}{\gamma_{m, \sigma}-\lambda_{m, n}^{+}}+\frac{\eta_{m}(l)}{i\left(\gamma_{m, \sigma}+\frac{K}{M a}\right) \mathcal{L}_{\ominus}\left(-\frac{K}{M a}\right)}\right) \\
& \overleftarrow{R}_{m, \sigma}=\frac{\left(\gamma_{m, \sigma}-\frac{K}{M a}\right)}{\sqrt{1-M a^{2}} J_{m}\left(K_{\sigma}^{m} R\right) \mathcal{L}_{\ominus}\left(-\gamma_{m, \sigma}\right)} \Delta_{m, \sigma} \times \\
&\left(\frac{M a\left(1+\gamma_{m, s} \frac{M a}{K}\right) \vec{E}_{m, s} J_{m}\left(K_{s}^{m} R\right)}{i Z\left(1-M a^{2}\right)\left(\gamma_{m, \sigma}+\gamma_{m, s}\right) \mathcal{L}_{\oplus}\left(\gamma_{m, s}\right)}+\sum_{n=0}^{\infty} \frac{P_{n}\left(-\lambda_{m, n}^{-}\right)}{\gamma_{m, \sigma}-\lambda_{m, n}^{-}} u_{\ominus}^{*}\left(-\lambda_{m, n}^{-}\right)\right. \\
&\left.\quad+\frac{\eta_{m}(0)}{i\left(\gamma_{m, \sigma}-\frac{K}{M a}\right) \mathcal{L}_{\oplus}\left(-\frac{K}{M a}\right)}+\eta_{m}(l) \sum_{n=0}^{\infty} \frac{R_{n}\left(-\lambda_{m, n}^{-}\right)}{\gamma_{m, \sigma}-\lambda_{m, n}^{-}}\right)
\end{aligned}
$$


Der folgende Faktor hat für die Grundmode einen anderen Wert als für die höheren:

$$
\Delta_{m, \sigma}=\left\{\begin{array}{cl}
-\frac{1}{K R} & , m \text { gerade, } \sigma=0 \\
-\frac{\left(K_{\sigma}^{m} R\right)^{2}}{\gamma_{m, \sigma} R\left(\left(K_{\sigma}^{m} R\right)^{2}-m^{2}\right)} & , \text { sonst }
\end{array}\right.
$$

Die berechneten Streufaktoren (in [10] Gleichungen (37) und (38)) beziehen sich auf das Feld des Geschwindigkeitspotentials $\Phi$. Was experimentell bestimmt wurde, sind die Streufaktoren des Wechseldruckfeldes. Gleichung (3) der Arbeit gibt darüber Auskunft, wie sich die beiden Felder und damit die Amplituden in einander umrechnen; bis auf einen uninteressanten globalen Vorfaktor ergibt sich für eine Einzelmode $X \exp (i \gamma x)$

$$
X_{\text {Druck }} \propto\left(\gamma+\frac{K}{M a}\right) X_{\Phi}
$$

Zum zweiten ist zu berücksichtigen, daß die Streufaktoren (C.48) und (C.49) auf die Ebene $x=0$, die Amplituden im stromab gelegenen Meßrohr bei BRANDES hingegen auf die Ebene $x=l$ bezogen sind, siehe Abb. 2.3 in [3].

Die experimentell ermittelten Druck-Transmissions- und -Reflexionsfaktoren $T_{\rightarrow}$, $T_{\leftarrow}, R_{\leftarrow}$ und $R_{\rightarrow}$ ergeben sich daher auf die folgende Weise $\left(\gamma_{0,0}=-K\right)$ aus den Streufaktoren (C.48) und (C.50):

$$
\begin{aligned}
& T_{\rightarrow}=e^{-i K l} \vec{T}_{0,0} \\
& R_{\leftarrow}=\frac{1+M a}{1-M a} \overleftarrow{R}_{0,0} \\
& T_{\leftarrow}=e^{-i K l} \frac{1-M a}{1+M a} \overleftarrow{R}_{0,0} \\
& R_{\rightarrow}=e^{-2 i K l} \vec{T}_{0,0}
\end{aligned}
$$




\section{Literaturverzeichnis}

[1] AurÉGan, Y. und M. Leroux: Failures in the discrete models for flow duct with perforations: an experimental investigation. J. Sound Vib., 265, 109-121, 2003. 1

[2] Brandes, M.: Optimierung eines Meßverfahrens zur Bestimmung von akustischen Parametern im durchströmten Rohr. Diplomarbeit, III. Physikal. Institut der Universität Göttingen, 1992. 2, 4

[3] BRAndes, M.: Schallverstärkung in Strömungskanälen mit resonanzartiger Wandauskleidung. Doktorarbeit, Math.-Nat. Fak. d. Georg-AugustUniversität zu Göttingen, 1997. 2, 4, 13, 31, 43, 68, 74, 87, 114

[4] Briggs, R. J.: Electron-Stream Interaction with Plasmas. Res. Monogr. No. 29. MIT Press Cambridge, Mass., 1964. 24, 88

[5] Doetsch, G.: Anleitung zum praktischen Gebrauch der LaplaceTransformation. R. Oldenbourg Verlag München, 1961. 110

[6] EnghardT, L.: Bestimmung akustischer Streufaktoren im durchströmten Rohr: Optimierung von Mikrofonanordnung und Meßprozedur. Diplomarbeit, III. Physikal. Institut der Universität Göttingen, 1992. 2, 4

[7] Grosser, J. und D. Ronneberger: Auf dem Weg zu einem akustisch regelbaren Strömungswiderstand. In: Fortschritte der Akustik DAGA 2003. 76

[8] Jones, D. S. und J. D. Morgan: A linear model of a finite amplitude Helmholtz instability. Proc. R. Soc. Lond. A, 338, 17-41, 1974. 24, 25, 89, 91

[9] JüschKe, M.: private Mitteilung. 87 
[10] Koch, W. und W. MöHRING: Eigensolutions for Liners in Uniform Mean Flow Ducts. AIAA Journal, 21(2), 200-213, Februar 1983. I, 1, 14, 37, 45, $68,73,74,76,78,83,87,100,101,102,104,105,106,108,110,114$

[11] Krause, M.: Schallausbreitung in Strömungskanälen mit kassettierter Wandauskleidung. Diplomarbeit, III. Physikal. Institut der Universität Göttingen, 1990. 2

[12] Landau, L. D. und E. M. Lifschitz: Lehrbuch der theoretischen Physik Bd. VI: Hydrodynamik. Akademie Verlag Berlin, 5. Auflage. 30, 100

[13] Lange, B. und D. Ronneberger: Active Noise Control by Use of an Aeroacoustic Instability. Acta Acustica united with Acustica, 89, 658-665, 2003. 2

[14] Mechel, F. P.: Schalldämpfung und Schallverstärkung in Luftströmungen durch absorbierend ausgekleidete Kanäle. Acustica, Vol. 10(3), 133-148, 1960. 1

[15] Nilsson, B.: private Mitteilung. 76

[16] Noble, B.: Methods Based on the Wiener-Hopf Technique. Pergamon Press, 1958. 69

[17] Rebel, J. und D. Ronneberger: The Effect of Shear Stress on the Propagation and Scattering of Sound in Flow Ducts. J. Sound Vib., 158(3), 469-496, 1992. 14, 59

[18] Rienstra, S. W.: Hydrodynamic Instabilities and Surface Waves in a Flow over an Impedance Wall. In: Symposium IUTAM Aéro et Hydro-Acoustique, 1985. 14, 20, 25

[19] Ronneberger, D.: private Mitteilung. 14

[20] Ronneberger, D.: Genaue Messung der Schalldämpfung und der Phasengeschwindigkeit in durchströmten Rohren im Hinblick auf die Wechselwirkung zwischen Schall und Turbulenz. Habilitationsschrift, Math.-Nat. Fak. d. Georg-August-Universität zu Göttingen, 1975. 2, 5

[21] Schlichting, H. und K. Gersten: Grenzschicht-Theorie. Springer-Verlag Berlin Heidelberg, 1997. 47 


\section{Danksagung}

Die vorliegende Doktorarbeit habe ich in der Forschungsgruppe von Prof. Ronneberger am Dritten Physikalischen Institut der Universität Göttingen angefertigt. Weder sah er ein Problem darin, mich als theoretischen Physiker in seine anwendungsorientierte Gruppe aufzunehmen, noch ließ er sich von meinem mathematischen Blickwinkel schrecken. Ich habe seine gänzlich andere Sicht auf physikalische Phänomene als inspirierend für meinen Arbeit empfunden.

Für die enge Betreuung dieser Arbeit gilt ihm mein herzlicher Dank.

Danken möchte ich auch meinen Arbeitskollegen und den Mitarbeitern des Institutes für Hilfe in vielerlei Hinsicht und das angenehme Arbeitsatmosphäre, ganz besonders Matthias Jüschke fürs Korrekturlesen und Reinhard Geisler für seine Tips bei der Konvertierung ins pdf-Format. Herausheben muß ich Dr. Michael Hartmann, der nach Abschluß seiner Promotion noch einmal an das Institut zurückkehrte, um sein Programm zur direkten numerischen Simulation turbulenter Kanalströmungen für dieses Projekt zu erweitern.

Diese Promotion wurde vom Graduiertenkolleg „Strömungsinstabilitäten und Turbulenz" gefördert, dem ich zuletzt als assoziiertes Mitglied angehörte. 


\title{
Lebenslauf
}

\author{
Name: \\ Jakob Großer \\ geboren: \\ Eltern: \\ 23. September 1974 in Plauen \\ Staatsangehörigkeit \\ Monika und Lucian Großer \\ Familienstand: \\ deutsch \\ ledig \\ 1981-1985 \\ 1985-1989 \\ Polytechnische Oberschule Weischlitz \\ 1989 \\ 1989-1990 \\ 1990-1994 \\ 8. Juni 1994 \\ WS 1994/95-SS 1999 \\ 6. Juli 1999 \\ WS 1999/00-SS 2003 \\ Polytechnische Oberschule Reuth \\ Spezialschule mathematisch-naturwissen- \\ schaftlich-technischer Richtung Chemnitz \\ Karolinengymnasium Frankenthal/Pfalz \\ Gymnasium Ulricianum Aurich \\ Allgemeine Hochschulreife \\ Physikstudium an der Albert-Ludwigs- \\ Universität Freiburg, Stipendiat der Stu- \\ dienstiftung des Deutschen Volkes \\ Physik-Diplom der Universität Freiburg \\ Promotionsstudium an der Georg-August- \\ Universität Göttingen, Stipendiat des \\ Graduiertenkollegs „Strömungsinstabilitäten \\ und Turbulenz" \\ April 2002-Sept. 2003 \\ Wissenschaftlicher Mitarbeiter am Dritten \\ Physikalischen Institut
}

